\title{
HE
}

6652

.A1 1921X NPM 



\section{DOMINION OF CANADA}

\section{REPORT}

RELATING TO

\section{MALL SUBSIDIES AND STEAMSHIP SUBVENTIONS}

AS CONTROLLED BY THE

\section{DEPARTMENT OF TRADE AND COMMERCE}

FOR THE

Fiscal Year ending $\mathbb{M}_{\text {arch }} 31,1920$, with Traffic Returns, etc., to

December 31, 1920.

This Report is published as a Supplement to the Annual Report of the Deputy Minister. and was formerly published as Part VI of the Annual Report of the Department.

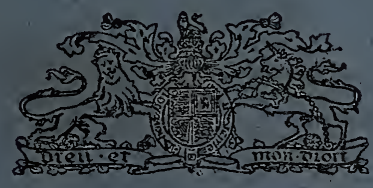

OTTAWA

THOMAS MULVEY

PRINTER TO THE KING'S MOST EXCELLENT MAJESTY 1921.

[No. 10a-1921.] Price, 10 cents. 


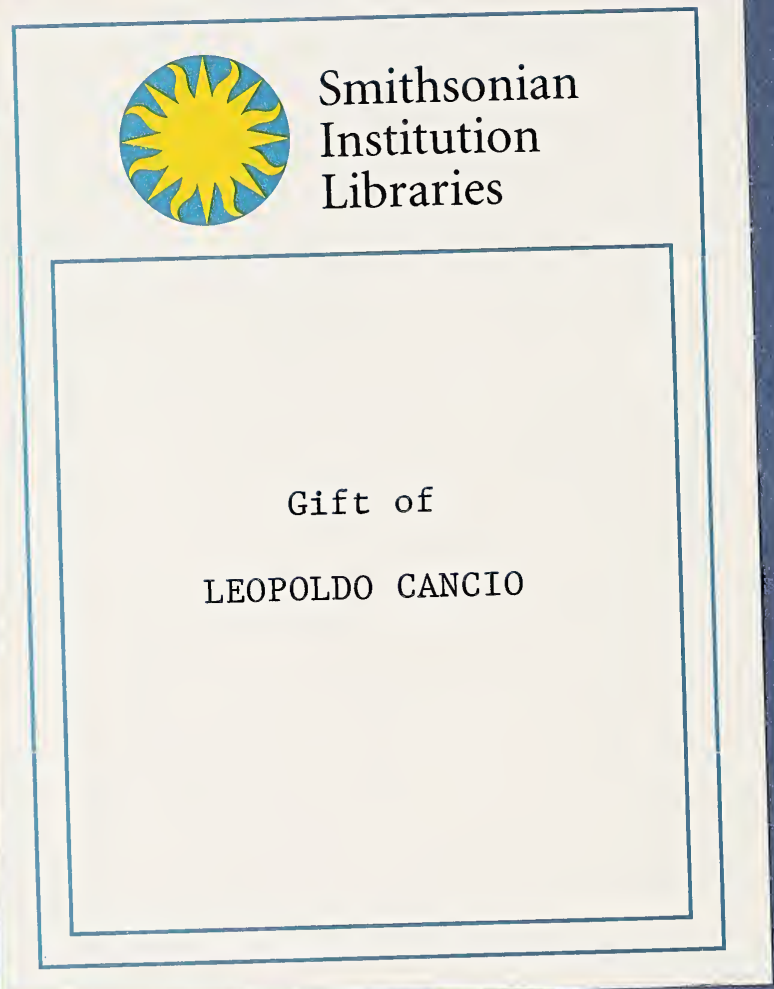




\title{
DOMINION OF CANADA
}

\author{
REPOR T
}

RELATING TO

\section{MALL SUBSIDIES AND STEAMSHIP SUBVENTIONS}

\author{
AS CONTROLLED BY THE \\ DEPARTMENT OF TRADE AND COMMERCE \\ FOR THE
}

Fiscal Year ending March 31,1920 , with Traffic Returns, etc., to December 31, 1920.

This Report is published as a Supplement to the Annual Report of the Deputy Minister. end was formerly published as Part VI of the Annual Report of the Department.

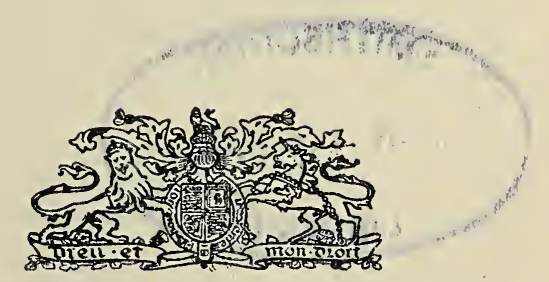

OTTAWA

THOMAS MULVEY

PRINTER TO THE KING'S MOST EXCELLENT MAJESTY

1921. 


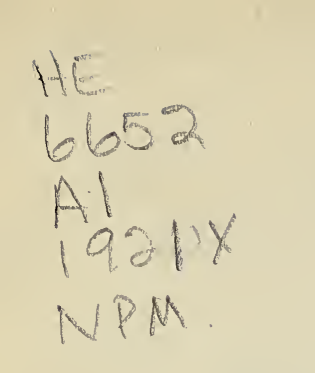


Explanation of Estimates for the year ending March 31, 1922, as compared with those for the year ending March 31, 1921, with statements of services rendered and expenditures to December 31, 1920, on account of Mail Subsidies and Steamship Subventions.

\section{XVII.-MAIL SUBSIDIES AND STEAMSHIP SUBVENTIONS}

Amount to be voted, $\$ 1,033,800.66$.

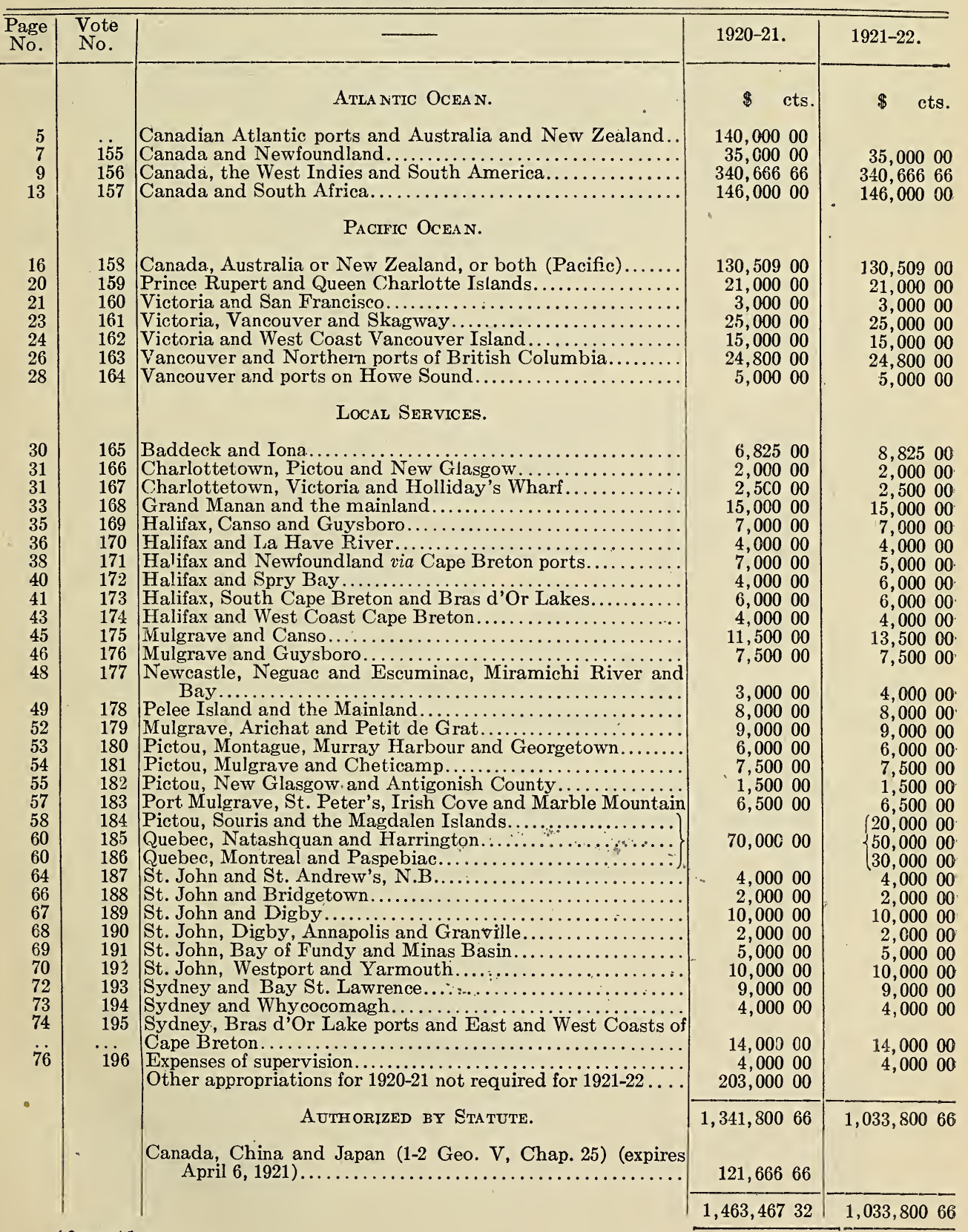





\section{ATLANTIC OCEAN SERVICES}

\section{CANADIAN ATLANTIC PORTS AND AUSTRALIA AND NEW ZEALAND}

NEW ZEALAND SHIPPING CO., LTD.

Contract No. 46.

T. \& C. File No. 25729.

Canadian Atlantic ports and Australia and New Zealand, sieam service between-

$$
\begin{aligned}
& 1920-21 \ldots \ldots \ldots \ldots \ldots \ldots \text {. . . . . . . . . . . . . } \$ 140,000 \\
& 1921-22 \ldots \ldots \ldots \text {. . . . . . . . . . . . . . . . . . . . . . . . . . . }
\end{aligned}
$$

Contractors.-New Zealand Shipping Co., Ltd., of London, England. (Canadian. address: 213 Board of Trade building, Montreal, Que.) .

Contract dated.-June 5, 1920.

Duration of Contract.-Opening of navigation, 1920, until March 31, 1921.

Service.-Approximately monthly. This is an outward service only, and these steamers do not return to Canada directly.

Ports of Call.-(a) During the season of open navigation on the St: Lawrence, from Montreal to the ports, or any two of the ports, of Auckland, Wellington, Lyttleton and Dunedin, N.Z., and the ports of Melbourne and Srdney, Aus., calling at such other ports in New Zealand or Australia as the contractors may desire.

(b) During the season of closed navigation on the St. Lawrence, from St. Johin, N.B., calling at Halifax, and thence proceeding to the ports in New Zealand and Australia mentioned in paragraph $(a)$.

Speed and Capacity Required.-10 knots, each ship to be capable of carrying 7,500 tons at 40 cubic feet to the ton.

Subsidy. $-\$ 140,000$ per annum, payable at the rate of $\$ 11,666.66$ for each trip.

Cold storage.-Steamers to be fitted with reasonable cold storage accommodation should occasion warrant.

Deduction for Short Cargo.-An average cargo of 5,000 tons on each monthly trip is to be carried thronghout the year; and $\$ 2$ is to be deducted from the subsidy at the end of each year for every ton short of the total measurement or weight which should have been carried upon such number of sailings as mav have been performed. based upon the said 5,000 tons per voyage.

Mails.-To be carried free.

Trade Commissioners.-To be carried free.

Government Railway Clause.-Included.

DISTANICES.

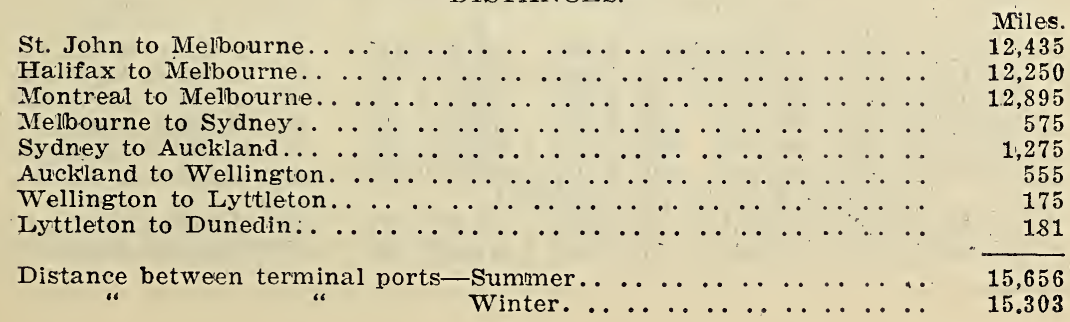


DESCRIPTIONS OF VESSELS EMPLOYED.

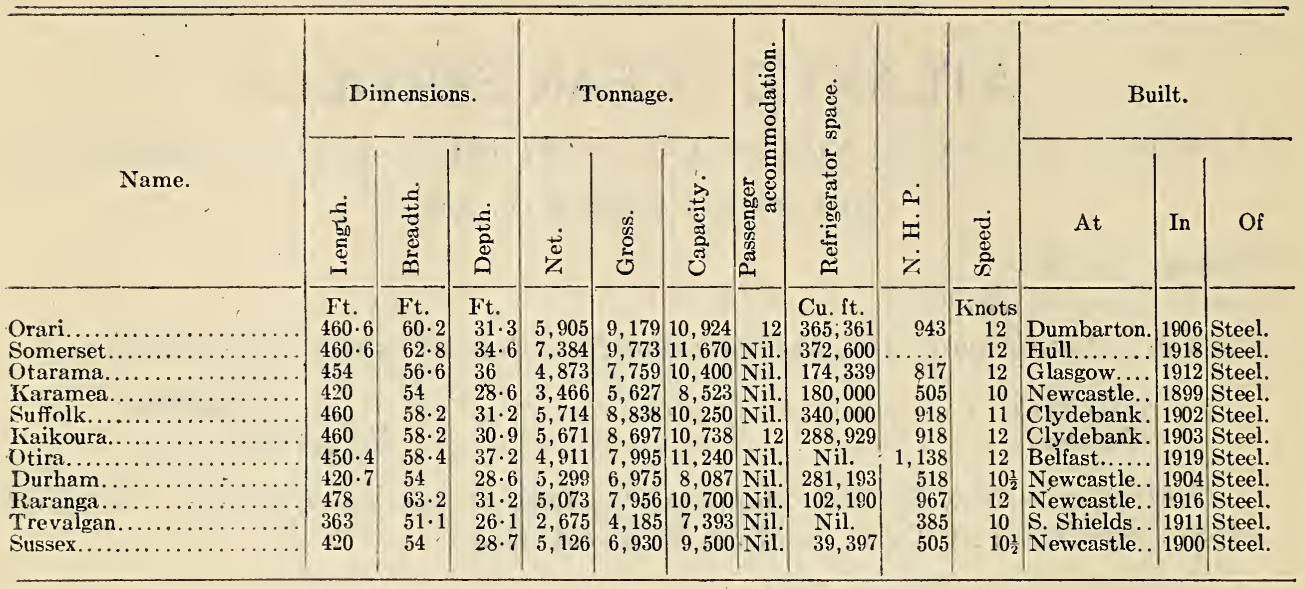

TRAFFIC RETURNS.

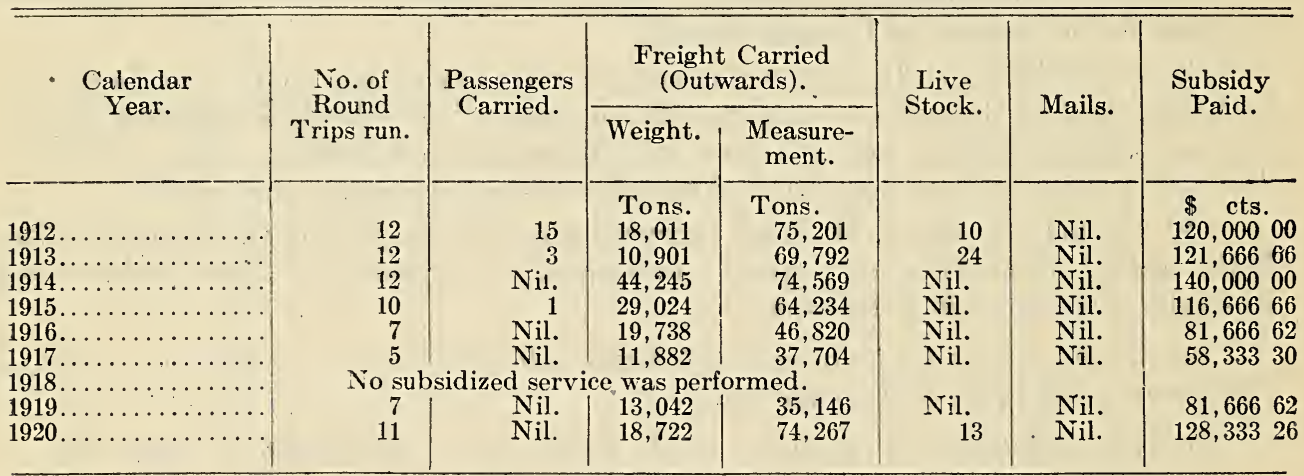

No Inward passengers or cargo are carried.

ORIGIN, QUANTITY AND VALUE OF CARGO EXPORTED FROM CANADA.

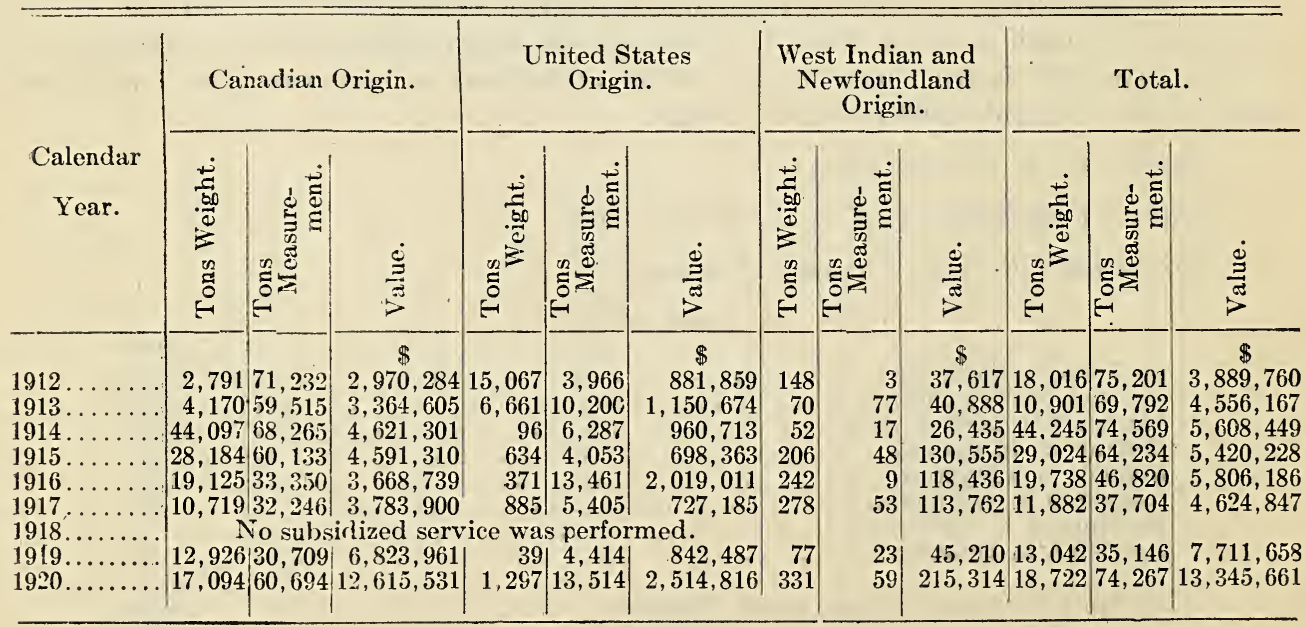


SESSIONAL PAPER No. $10 a$

\section{PRINCIPAL ARTICLES EXPORTED}

Of Canadian Origin.-Nails and staples, pig iron, steel sheets, plaster, insulated cable, enamelware, automobiles and parts thereof, calcium carbide, chairs, newsprint paper, wall paper, wrapping paper, other paper, sole leather, agricultural implements, rubber goods, iron pipe, hardware, clothes pins, seed, fruit jars, corsets, cereal foods, wire, pianos, asbestos fibre, paint and varnish.

Of United States Origin.-Automobiles and parts thereof, organs, wallboard, wrapping paper, tractors, motor cycles, pianos and batteries.

Of West Indian Origin.-Cocoa, pimento, ginger and lime juice.

\section{CANADA AND NEWFOUNDLAND}

The Reid Newfoundland Company, Ltd.

Contract No. 60 .

T. \& C. File No: 23660.

Vote 155.-Canada and Newfoundland.-Steam service or services between-

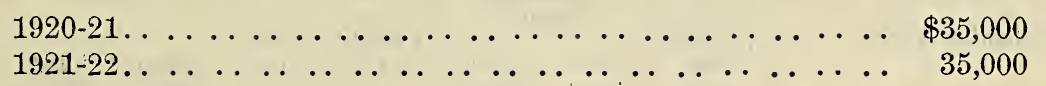

Contractors.-The Reid Newfoundland Company, Ltd., of St. John's, Nfld.

Contract Dated.-May 23, 1919.

Duration of Contract.-April 1, 1919, to March 31, 1920. (This contract has not been renewed yet).

Service.-One complete round trip each day, except Sunday, between North Sydney and Port aux Basques.

Should Port aux Basques or North Sydney be blocked with ice at any time, the service may during such period, at the option of the Contractors, be performed to Placentia, Nfld., and Louisburg, N.S., respectively.

Ports of Call.--North Sydney (or Louisburg), N.S., and Port aux Basques (or Placentia), Nfld.

Speed required.-Not stated.

Subsidy.-At the rate of $\$ 70,000$ per annum, until March 31, 1920, payable quarterly, on June 30, September 30, December 31, and March 31.

Mails.-To be carried free.

Canadian Trade Commissioners.-To be carried free.

Government Wharves.-Steamers are required to call at Government wharves whenever possible.

\section{DISTANCES}

Miles

North Sydney to Port aux Basques. . . . . . . . . . . 101

Louisburg to Placentia.. . . . . . . . . . . . . 250

North Sydney to St. John's. . . . . . . . . . . . 300 
DESCRIPTION OF VESSELS EMPLOYED.

\begin{tabular}{|c|c|c|c|c|c|c|c|c|c|c|c|c|c|c|c|}
\hline \multirow[b]{2}{*}{ Name. } & \multicolumn{3}{|c|}{ Dimensions. } & \multicolumn{3}{|c|}{ Tonnage. } & \multicolumn{3}{|c|}{$\begin{array}{l}\text { Passenger } \\
\text { Accom- } \\
\text { modation. }\end{array}$} & \multirow{2}{*}{ 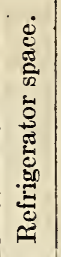 } & \multirow[b]{2}{*}{$\begin{array}{l}\dot{a} \\
\dot{z} \\
\dot{z}\end{array}$} & \multirow[b]{2}{*}{ 总 } & \multicolumn{3}{|c|}{ Built. } \\
\hline & 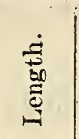 & 密 & 远 & 苛 & $\begin{array}{l}\dot{0} \\
\dot{0} \\
\dot{0} \\
\dot{0}\end{array}$ & 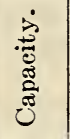 & $\begin{array}{l}\dot{0} \\
\overrightarrow{0} \\
\vec{J} \\
\overrightarrow{0} \\
\overrightarrow{0} \\
\overrightarrow{0}\end{array}$ & 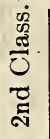 & 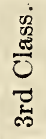 & & & & At & In & Of \\
\hline $\begin{array}{l}\text { Kyle......... } \\
\text { Sagona....... } \\
\text { Glencoe.... }\end{array}$ & $\begin{array}{r}\text { Ft. } \\
220 \\
175 \\
208\end{array}$ & $\begin{array}{c}\text { Ft. } \\
32 \cdot 3 \\
28 \cdot 3 \\
30 \cdot 1\end{array}$ & $\begin{array}{l}\text { Ft. } \\
18 \cdot 3 \\
20 \cdot 3 \\
16 \cdot 7\end{array}$ & $\begin{array}{l}548 \\
420 \\
336\end{array}$ & $\begin{array}{r}1,055 \\
808 \\
767\end{array}$ & 3 & $\begin{array}{l}68 \\
40 \\
42\end{array}$ & $\begin{array}{r}160 \\
77 \\
125\end{array}$ & & $\begin{array}{l}\text { c.ft. } \\
\text { Nil. } \\
\text { Nil. } \\
\text { Nil. }\end{array}$ & $\begin{array}{l}263 \\
136 \\
185\end{array}$ & $\begin{array}{c}\mathrm{Kts} \\
12 \\
11 \\
13\end{array}$ & $\begin{array}{l}\text { Newcastle.. } \\
\text { Dundee..... } \\
\text { Pointhouse. }\end{array}$ & $\left|\begin{array}{l}1913 \\
1914 \\
1899\end{array}\right|$ & $\begin{array}{l}\text { Steel. } \\
\text { Steel. } \\
\text { Steel. }\end{array}$ \\
\hline
\end{tabular}

TRAFFIC RETURNS.

No service was run during 1907.

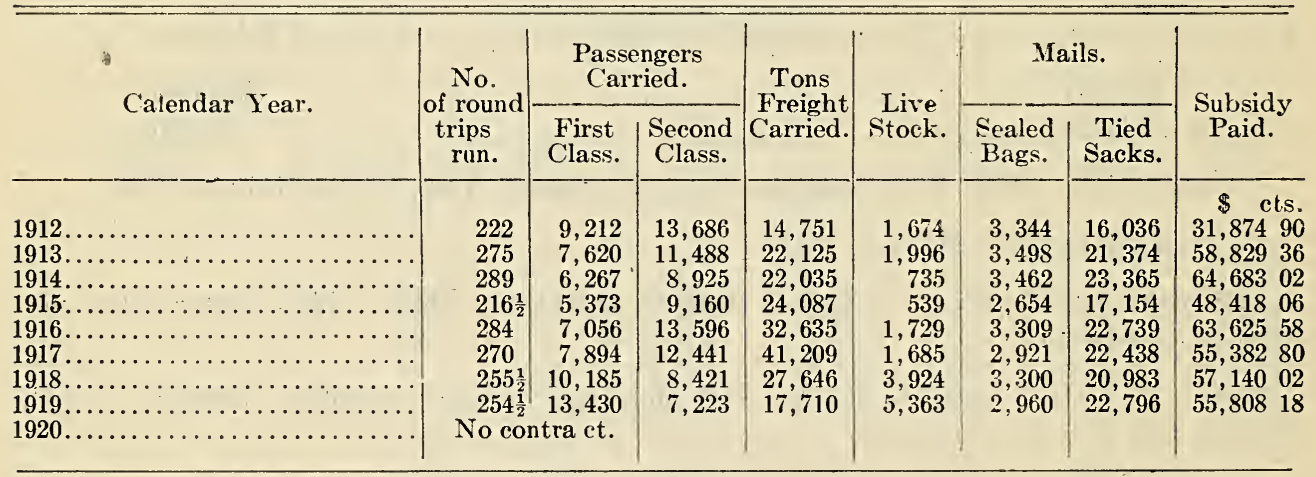

ORIGIN, QUANTITY AND VALUE OF CARGO EXPORTED FROM CANADA.

(Including Live Stock.)

\begin{tabular}{|c|c|c|c|c|c|c|c|c|c|}
\hline \multirow{2}{*}{$\begin{array}{l}\text { Calendar } \\
\text { Year. }\end{array}$} & \multicolumn{3}{|c|}{ Canadian Origin. } & \multicolumn{3}{|c|}{ United States Origin. } & \multicolumn{3}{|c|}{ Total. } \\
\hline & $\begin{array}{c}\text { Tons } \\
\text { weight }\end{array}$ & $\begin{array}{l}\text { Tons } \\
\text { measure- } \\
\text { ment. }\end{array}$ & Value. & $\begin{array}{c}\text { Tons } \\
\text { weight. }\end{array}$ & $\begin{array}{c}\text { Tons } \\
\text { measure- } \\
\text { ment. }\end{array}$ & Value. & $\begin{array}{c}\text { Tons } \\
\text { weight. }\end{array}$ & $\left|\begin{array}{c}\text { Tons } \\
\text { measure- } \\
\text { ment. }\end{array}\right|$ & Value. \\
\hline $\begin{array}{l}1912 \ldots \ldots \\
1913 \ldots \ldots \\
1914 \ldots \ldots \\
1915 \ldots \ldots \\
1916 \ldots \ldots \\
1917 \ldots \ldots \\
1918 \ldots \ldots \\
1919 \ldots \ldots \\
1920 \ldots \ldots\end{array}$ & $\begin{array}{r}12,224 \\
17,372 \\
13,286 \\
16,510 \\
16,692 \\
26,838 \\
15,626 \\
12,669 \\
\text { No }\end{array}$ & $\begin{array}{l}\text { Nil. } \\
\text { Nil. } \\
\text { Nil. } \\
\text { Nil. } \\
\text { Ni.. } \\
\text { Nil. } \\
\text { Nil. } \\
\text { Nil. } \\
\text { ontract. }\end{array}$ & $\begin{array}{c}\$ \\
894,220 \\
981,369 \\
858,605 \\
1,108,876 \\
1,559,228 \\
4,165,668 \\
2,392,697 \\
1,911,162\end{array}$ & $\begin{array}{r}1,821 \\
3,694 \\
7,651 \\
6,491 \\
9,534 \\
13,192 \\
7,081 \\
2,408\end{array}$ & $\begin{array}{l}\text { Nil. } \\
\text { Nil. } \\
\text { Nil. } \\
\text { Nil. } \\
\text { Nil. } \\
\text { Nii. } \\
\text { Nil. } \\
\text { Nil. }\end{array}$ & $\begin{array}{c}\$ \\
317,936 \\
288,222 \\
587,196 \\
643,885 \\
1,280,032 \\
2,585,724 \\
2,182,497 \\
395,107\end{array}$ & $\begin{array}{l}14,046 \\
21,066 \\
20,937 \\
23,001 \\
26,226 \\
40,030 \\
22,707 \\
15,077\end{array}$ & $\begin{array}{l}\text { Nil. } \\
\text { Nil. } \\
\text { Nil. } \\
\text { Nil. } \\
\text { Nil. } \\
\text { Nil. } \\
\text { Nil. } \\
\text { Nil. }\end{array}$ & $\begin{array}{c}\$ \\
1,212,156 \\
1,269,591 \\
1,445,801 \\
1,752,761 \\
2,839,260 \\
6,751,392 \\
4,575,194 \\
2,806,269\end{array}$ \\
\hline
\end{tabular}




\section{PRINCIPAL ARTICLES EXPORTED}

Of Canadian Origin.-Flour, oats, hay, bran, feed, potatoes, live stock, beef, pork, fresh meal, condensed milk, machinery, lard, yeast cake, pig iron, roofing and cement.

Of United States Origin.-Flour, meal, oats, dried fruit, pork, beef, leather, oil, organs, soap, beans, rice, roofing, tobacco, sugar and machinery.

\section{CANADA, THE WEST INDIES AND SOUTH AMERICA}

\section{The Royal Mail Stena Packet Company}

Contract No. 9.

T. \& C. File No. 24215.

Vote 156.-Canada and the West Indies or South America, or both, steam service between-

$$
\begin{aligned}
& 1920-21 \ldots \ldots \ldots \ldots \ldots \ldots \text {. . . . . . . . . . . . . \$ } \$ 340,66666 \\
& 1921-22 \ldots \ldots \ldots \ldots \ldots \text {. . . . . . . . . . . . . . . 340,666 } 66
\end{aligned}
$$

Contractors.-The Royal Mail Steam Packet Company, of London, England.

(Canadian representative: John Allsop, 59 Granville St., Halifax, N.S.)

(Freight and passenger agents: Pickford and Black, Halifax, N.S.)

Contract. Dated.-September 11, 1919.

Duration of Contract.-November 1, 1919, to October 31, 1920. (This has been continued by Order in Council until October 31, 1921.)

Service and Ports of Call.-Commencing from St. John, N.B., sailing thence to Halifax, N.S., and sailing thence to Georgetown, British Guiana, every fourteen days:-

Calling at the following islands: Bermuda, St. Kitts, Antigua, Montserrat, Dominica, St. Lucia, St. Vincent, Grenada, Barbados, and Trinidad, and returning from Georgetown to St. John, calling at all the aforesaid islands, in reversed order.

This itinerary may be subject to any change which may be mutually agreed upon between the minister and the contractors.

Speed required.-11 knots.

Subsidy.- $£ 70,000(\$ 340,666.66)$ per annum, based on payments of $£ 2,6926 \mathrm{~s} .2 \mathrm{~d}$, $(\$ 13,102.56)$ for each complete round royage, payable on the last day of each month.

Canadian Trade Commissioners.-To be carried free.

Mails.-To be carried free.

Government Railway Clause.-Included.

Freight charges from St. John to Halifax.-The contractors are required, at their own expense, when so required by consignors, to pay the freight charges by rail from St. John to Halifax on butter, cheese, and fruit intended for shipment by the contractors' steamships.

Delay at Ports.-The contractors must make every reasonable effort to avoid. undue delay at Canadian or West Indian ports.

Development of Trade.-The contractors must use their utmost endeavour tc develop the cargo and passenger trade between Canada and the British West Indies by means of reasonable advertising and regular solicitation through agents. 
Through rates of Freight.-The contractors must use their best endeavours to arrange through rates of freight between inland points in Canada and the various ports of call referred to in this contract in the British West Indies and Central and South America.

Transfer by connecting lines.-As the design of this agreement is to give regular fortnightly communication both ways to all the ports previously mentioned, arrangements must be made for the transport of freight and passengers on all voyages south bound and north bound by transfer to the lines of the contractors' steamers conducting the insular service from and to Trinidad, and at the rates obtaining for the direct service.

No discrimination.-No discrimination of any kind as regards freight and passenger rates may be made in favour of any merchant, shipper or importer in any one of the British colonies referred to herein, as against any other merchant, shipper or importer in the same colony.

Through Bills of Lading.-Through bills of lading must be issued from any Canadian point of shipment to any port in Central or South America, which is a regular port of call for any of the steamships employed or controlled by the contractors on other services, and which make regular connections with the service herein contracted for.

DISTANICES.

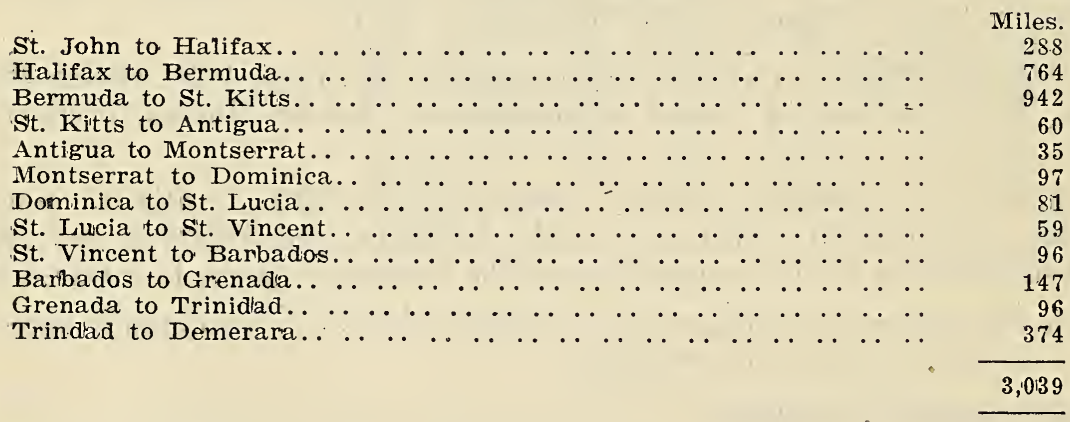

DESCRIPTION OF VESSELS EMPLOYED.

\begin{tabular}{|c|c|c|c|c|c|c|c|c|c|c|c|c|c|c|c|}
\hline \multirow[b]{2}{*}{ Name. } & \multicolumn{3}{|c|}{ Dimensions. } & \multicolumn{3}{|c|}{ Tonnage. } & \multicolumn{3}{|c|}{$\begin{array}{c}\text { Passenger } \\
\text { Accom- } \\
\text { modation. }\end{array}$} & \multirow{2}{*}{ 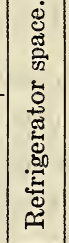 } & \multirow[b]{2}{*}{$\mid \begin{array}{l}2 i \\
\dot{4} \\
\dot{z}\end{array}$} & \multirow[b]{2}{*}{ 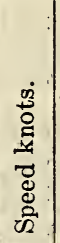 } & \multicolumn{3}{|c|}{ Built. } \\
\hline & 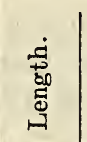 & 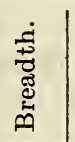 & 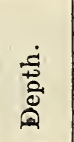 & $\begin{array}{l}\overrightarrow{0} \\
\ddot{z}\end{array}$ & . & 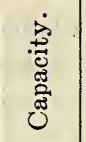 & 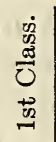 & 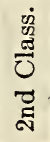 & $\begin{array}{l}\dot{0} \\
\text { 离 } \\
\dot{0} \\
\dot{0} \\
\dot{b}\end{array}$ & & & & At & In & Of \\
\hline $\begin{array}{l}\text { Chignecto... } \\
\text { Chaleur... } \\
\text { Caraquet... } \\
\text { Chaudière. }\end{array}$ & $\begin{array}{l}\mathrm{Ft} . \\
400 \cdot 5 \\
400 \cdot 5 \\
400 \cdot 5 \\
370\end{array}$ & \begin{tabular}{l|} 
Ft. \\
$47 \cdot 2$ \\
$47 \cdot 2$ \\
$47 \cdot 3$ \\
$45 \cdot 9$
\end{tabular} & $\begin{array}{l}\text { Ft. } \\
31 \cdot 1 \\
31 \cdot 1 \\
31 \cdot 1 \\
25\end{array}$ & $\begin{array}{l}2,999 \\
2,994 \\
2,975 \\
2,499\end{array}$ & $\begin{array}{r}4 \cdot 744 \\
.4,746 \\
4,889 \\
4,019\end{array}$ & $\begin{array}{l}5,567 \\
5,574 \\
5,129 \\
4,726\end{array}$ & $\begin{array}{l}41 \\
41 \\
40 \\
50\end{array}$ & $\begin{array}{l}60 \\
60 \\
72 \\
64\end{array}$ & $\begin{array}{l}90 \\
90 \\
80 \\
76\end{array}$ & $\begin{array}{r}\text { c.ft. } \\
4988 \\
5288 \\
5565 \\
900\end{array}$ & $\begin{array}{l}418 \\
418 \\
418 \\
584\end{array}$ & $\begin{array}{c}\mathrm{Kts} \\
11 \\
11 \\
11 \\
12\end{array}$ & $\begin{array}{l}\text { Belfast... } \\
\text { Belfast... } \\
\text { Belfast... } \\
\text { Middıebo }\end{array}$ & $\begin{array}{l}1893 \\
1893 \\
1894 \\
1899\end{array}$ & $\begin{array}{l}\text { Steel. } \\
\text { Steel. } \\
\text { Steel. } \\
\text { Steel. }\end{array}$ \\
\hline
\end{tabular}


SESSIONAL PAPER No. 10a

TRAFFIC RETURNS.

\begin{tabular}{|c|c|c|c|c|c|c|c|c|c|c|c|}
\hline $\begin{array}{c}\text { Calendar } \\
\text { Year. }\end{array}$ & $\begin{array}{c}\text { No. of } \\
\text { round } \\
\text { trips } \\
\text { run. }\end{array}$ & \multicolumn{4}{|c|}{$\begin{array}{l}\text { Number } \\
\text { of Passengers } \\
\text { Carried. }\end{array}$} & \multicolumn{2}{|c|}{$\begin{array}{l}\text { Tons } \\
\text { of Freight } \\
\text { Carried. }\end{array}$} & \multirow[t]{2}{*}{ Live Stock. } & \multicolumn{2}{|c|}{ Mails. } & $\begin{array}{l}\text { Subsidy } \\
\text { Paid. }\end{array}$ \\
\hline i & $\gamma$ & 1st & Class. & $\begin{array}{l}\text { 2nd } \\
\text { Class. }\end{array}$ & $\begin{array}{l}\text { 3rd } \\
\text { Class. }\end{array}$ & $\begin{array}{c}\text { Tons } \\
\text { Weight. }\end{array}$ & $\begin{array}{c}\text { Tons } \\
\text { Measure- } \\
\text { ment. }\end{array}$ & & $\begin{array}{l}\text { Lock } \\
\text { Bags. }\end{array}$ & $\begin{array}{l}\text { Tied } \\
\text { Sacks. }\end{array}$ & $\$$ cts. \\
\hline $1912 \ldots \ldots$ & 30 & & 1,090 & 1,269 & 1,048 & 65,552 & 86,253 & 106 & 168 & 2,568 & 87,61386 \\
\hline $1913 \ldots \ldots$ & $28 \frac{1}{2}$ & & 887 & 827 & 1,016 & 52,313 & 70,209 & 44 & 144 & 2,396 & $131,737 \quad 12$ \\
\hline $1914 .$. & 26 & F & 1,080 & 465 & 1,742 & 52,320 & 90,398 & 48 & 705 & 1,627 & 330,89733 \\
\hline $1915 .$. & 26 & & 959 & 786 & 2,727 & 78,414 & 94,781 & 34 & 518 & 3,472 & 340,66656 \\
\hline $1916 \ldots .$. & 26 & & 1,459 & 461 & 3,948 & 100,883 & 127,631 & 31 & 1,690 & 3,146 & $340,666 \quad 66$ \\
\hline $1917 \ldots$ & 25 & & 1,253 & 422 & 2,256 & 94,042 & 99,504 & 32 & 3,421 & 1,970 & 334,11538 \\
\hline $1918 .$. & 16 & & 1,344 & 389 & 1,064 & 70,691 & 72,370 & 86 & 2,710 & 1,255 & 209,64096 \\
\hline 1919. & 26 & $\begin{array}{l}\text { In } \\
\text { Out }\end{array}$ & $\begin{array}{l}1,668 \\
1,486\end{array}$ & $\begin{array}{l}573 \\
527\end{array}$ & $\begin{array}{r}1,411 \\
460\end{array}$ & $\begin{array}{r}89,492 \\
4,398\end{array}$ & $\begin{array}{r}3,376 \\
106,937\end{array}$ & $\begin{array}{r}2 \\
160\end{array}$ & $\begin{array}{l}1,069 \\
1,109\end{array}$ & $\begin{array}{l}2,056 \\
2,036\end{array}$ & 340,66656 \\
\hline Total.... & $\ldots$ & & 3,154 & 1,100 & 1,871 & 93,890 & 110,313 & 162 & 2,178 & 4,092 & \\
\hline $1920 \ldots$ & 26 & $\begin{array}{l}\text { In } \\
\text { Out }\end{array}$ & $\begin{array}{l}1,590 \\
1,417\end{array}$ & $\begin{array}{l}709 \\
336\end{array}$ & $\begin{array}{r}1.247 \\
619\end{array}$ & $\begin{array}{r}61,877 \\
7,960\end{array}$ & $\begin{array}{r}5,591 \\
105,330\end{array}$ & $\begin{array}{r}\text { Nil. } \\
107\end{array}$ & $\begin{array}{l}1,268 \\
1,254\end{array}$ & $\begin{array}{l}1,652 \\
1,931\end{array}$ & 340,66656 \\
\hline Total. & $\ldots$ & & 3,007 & 1,045 & 1,866 & 69,837 & 110,921 & 107 & 2,522 & 3,583 & \\
\hline
\end{tabular}
N.S.

From 1907 to Nov. 23, 1913, this service was performed by Méssrs. Pickford and Black, of Halifax,

Two sailings from Canada were made by the Royal Mail Steam Packet Company's steamers in December, 1913. Their traffic returns are included in the figures given above for 1913 . 
11 GEORGE V, A. 1921

ORIGIN, QUANTITY AND VALUE OF CARGO EXPORTED FROM CANADA.

(Including Live Stock.)

\begin{tabular}{|c|c|c|c|c|c|c|c|c|c|c|}
\hline \multirow{2}{*}{$\begin{array}{c}\text { Calen- } \\
\text { dar } \\
\text { Year. }\end{array}$} & \multirow[b]{2}{*}{ From. } & \multicolumn{3}{|c|}{ Canadian Origin. } & \multicolumn{3}{|c|}{ United States Origin. } & \multicolumn{3}{|c|}{ Total. } \\
\hline & & $\begin{array}{c}\text { Tons } \\
\text { Weight. }\end{array}$ & $\begin{array}{c}\text { Tons } \\
\text { Measure- } \\
\text { ment. }\end{array}$ & Value. & $\begin{array}{c}\text { Tons } \\
\text { Weight. }\end{array}$ & $\begin{array}{c}\text { Tons } \\
\text { Measure } \\
\text { ment. }\end{array}$ & Value. & \begin{tabular}{|l|} 
Tons \\
Weight.
\end{tabular} & $\begin{array}{c}\text { Tons } \\
\text { Measure- } \\
\text { ment. }\end{array}$ & Value. \\
\hline 1912. & $\begin{array}{l}\text { St. John... } \\
\text { Halifax... }\end{array}$ & $\begin{array}{l}1,377 \frac{1}{2} \\
5,044 \frac{3}{4}\end{array}$ & $\begin{array}{l}20,099 \frac{3}{4} \\
62,147 \frac{1}{2}\end{array}$ & $\begin{array}{c}\$ \\
383,536 \\
1,941,990 \\
\end{array}$ & & & $\$$ & $\begin{array}{l}1,377 \frac{1}{\frac{2}{2}} \\
5,044\end{array}$ & $\begin{array}{l}20,099 \frac{3}{4} \\
62,147 \frac{1}{2}\end{array}$ & $\begin{array}{r}\$ \\
383,536 \\
1,941,990\end{array}$ \\
\hline Total. & & $6,422 \frac{1}{2}$ & $82,247 \frac{1}{4}$ & $2,325,526$ & Nil. & Nil. & Nil. & $6,422 \frac{1}{4}$ & $82,247 \frac{1}{4}$ & $2,325,526$ \\
\hline 1913. & $\begin{array}{l}\text { St. John.. } \\
\text { Halifax... }\end{array}$ & $\begin{array}{l}2,137 \\
5,786\end{array}$ & $\begin{array}{l}13,038 \\
56,819\end{array}$ & $\begin{array}{r}259,381 \\
1,800,169\end{array}$ & & & $\cdots$ & & & $\begin{array}{r}259,381 \\
1,800,169\end{array}$ \\
\hline Total. & & 7,923 & 69,857 & $2,059,550$ & Nil. & Nil. & Nil. & 7,923 & 69,857 & $2,059,550$ \\
\hline 1914. & $\begin{array}{l}\text { St. John... } \\
\text { Halifax... }\end{array}$ & $\begin{array}{l}1,557 \\
2,951 \\
\end{array}$ & & $\begin{array}{r}234 \\
2,384 \\
\end{array}$ & & & &, 557 & & $\begin{array}{r}234, \\
2,384, \\
\end{array}$ \\
\hline Total. & & 4,508 & 90,374 & $2,618,264$ & Nil. & Nil. & Nil. & 4,508 & 90,374 & $2,618,264$ \\
\hline 1915 . & $\begin{array}{l}\text { St. John.. } \\
\text { Halifax... }\end{array}$ & $\begin{array}{l}2,031 \\
3,621\end{array}$ & & $\begin{array}{r}301,659 \\
3,206,176\end{array}$ & & & & $\begin{array}{l}2,031 \\
3,621\end{array}$ & $\begin{array}{l}12,362 \\
82,419\end{array}$ & $\begin{array}{r}301,659 \\
3,206,176\end{array}$ \\
\hline Total. & & 5,652 & 94,781 & $3,507,835$ & Nil. & Nil. & Nil. & 5,652 & 94,781 & $3,507,835$ \\
\hline 1916 . & $\begin{array}{l}\text { St. John.. } \\
\text { Halifax... }\end{array}$ & $\begin{array}{l}1,781 \\
2,812 \\
\end{array}$ & $\begin{array}{l}15,899 \\
96,907 \\
\end{array}$ & $\begin{array}{r}425,664 \\
4,272,628 \\
\end{array}$ & & & & $\begin{array}{l}1,781 \\
2,812 \\
\end{array}$ & & $\begin{array}{r}425,664 \\
4,272,628 \\
\end{array}$ \\
\hline Total. & & 4,593 & 112,806 & $4,698,292$ & Nil. & Nil. & Nil. & 593 & 112,806 & $4,698,292$ \\
\hline 1917. & $\begin{array}{l}\text { St. John.. } \\
\text { Halifax... }\end{array}$ & $\begin{array}{l}1,460 \\
5,071 \\
\end{array}$ & $\begin{array}{l}14,570 \\
89,820 \\
\end{array}$ & $\begin{array}{r}532,012 \\
5,726,139 \\
\end{array}$ & & & & $\begin{array}{l}1,460 \\
5,071 \\
\end{array}$ & $\begin{array}{l}14,570 \\
89,820 \\
\end{array}$ & $\begin{array}{r}532,012 \\
5,726,139 \\
\end{array}$ \\
\hline Total. & & 6,531 & 104,390 & $6,258,151$ & Nil. & Nil. & Nil. & 6,531 & 104,390 & $6,258,151$ \\
\hline 1918. & $\begin{array}{l}\text { St. John... } \\
\text { Halifax... }\end{array}$ & $\begin{array}{l}3,789 \\
2,295\end{array}$ & $\begin{array}{l}36,113 \\
36,257\end{array}$ & $\begin{array}{l}3,065,587 \\
3,194,007\end{array}$ & & & & $\begin{array}{l}3,789 \\
2,295\end{array}$ & $\begin{array}{l}36,113 \\
36,257\end{array}$ & $\begin{array}{l}3,065,587 \\
3,194,007\end{array}$ \\
\hline Total. & & 6,084 & 72,370 & $6,259,594$ & Nil. & Nil. & Nil. & 6,084 & 72,370 & $6,259,594$ \\
\hline 1915 & $\begin{array}{l}\text { St. John... } \\
\text { Halifax... }\end{array}$ & $\begin{array}{r}829 \\
3,569 \\
\end{array}$ & $\begin{array}{l}19,378 \\
87,559 \\
\end{array}$ & $\begin{array}{r}1,276,582 \\
6,836,810 \\
\end{array}$ & & & & $\begin{array}{r}829 \\
3,569 \\
\end{array}$ & $\begin{array}{l}19,378 \\
87,559 \\
\end{array}$ & $\begin{array}{l}1,276 \\
6,836 \\
\end{array}$ \\
\hline Total. & & 4,398 & 106,937 & $8,113,392$ & Nil. & Nil. & Nil. & 4,398 & 106,937 & $8,113,392$ \\
\hline 1920 . & $\begin{array}{l}\text { St. John... } \\
\text { Halifax... }\end{array}$ & $\begin{array}{l}1,320 \\
6,640 \\
\end{array}$ & $\begin{array}{l}20,894 \\
84,436 \\
\end{array}$ & $\begin{array}{r}1,477,800 \\
8,312,749 \\
\end{array}$ & & & & $\begin{array}{l}1,320 \\
6,640 \\
\end{array}$ & $\begin{array}{l}20,894 \\
84,436 \\
\end{array}$ & $\begin{array}{r}1,477,800 \\
8,312,749 \\
\end{array}$ \\
\hline Total.. & & 7,960 & 105,330 & $9,790,549$ & Nil. & Nil. & Nil. & 7,960 & 105,330 & $9,790,549$ \\
\hline
\end{tabular}


SESSIONAL PAPER No. 10a

\section{Principal Articles Exported frojl Canada}

All of Canadian Origin.-Fish, canned goods, flour, feed, meal, oilmeal, hay, oats, cheese, butter, eggs, apples, potatoes, split peas, vegetables, groceries, beef, live stock, mineral water, tea, soap, sulphate of ammonia, fertilizer, lumber, shingles, shooks, laths, furniture, chairs, brooms, brushes, stores, trunks, rope, cordage, nails, paper, and biscuits.

\section{CANADA AND SOUTH AFRICA \\ Elder Dempster and Co., Itd.}

Contract No. 2.

T. \& C. File No. 25776 .

Vote 15\%.-Canada and South Africa, steam service between--

$$
\begin{aligned}
& 1920-21 \ldots \ldots \ldots \ldots \ldots 146,000
\end{aligned}
$$

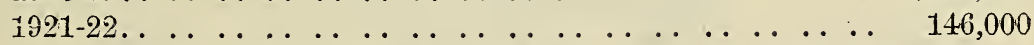

Contractors.-Elder Dempster \& Co., Ltd., 133 Board of Trade Building, Montreal, Que. (Head office: 4 S.t. Mary Axe., London, E.C., England.)

Contract dated.-June 9, 1920.

Duration of Contract.-April 1, 1920, to March 31, 1921.

Service.-Monthly, during the first fifteen days of each month.

Ports of Call.-From Montreal, calling at Quebec, at the option of the contractors; and during the months of September, October and November at Halifax, and, at the option of the contractors, at other Canadian ports during the season of open navigation on the St. Lawrence; and, during closed navigation on the St. Lawrence, from St. John, calling at Halifax, and, at the option of the contractors, at other Canadian ports; proceeding direct to Cape Town and not less than two other South African ports.

Speed required.-10 knots.

Subsidy. $\$ 146,000$ per annum, payable quarterly. coaling.

Coaling.-Steamers mav call at any Canadian port solely for the purpose of

\section{Government Railway Clause.-Included.}

Cold Storage.-There must be accommodation for not less than 200 tons of cargo in cold storage on each ship. The contractors must provide such further cold storage accommodation as may be needed from time to time.

Additional Vessels.-The contractors agree to provide additional vessels when necessary to meet the requirements of the trade offered.

Mails.-To be carried free.

Canadian Trade Commissioners.-To be carried free.

Supervision of handling.-The handling, loading, stowing and unloading of any fruit or perishable products carried by the said vessels shall be subject to and under the supervision of any cargo inspector or other officer appointed for that purpose, should the Minister of Agriculture for Canadn deem it advisable. 
11 GEORGE V., A. 1921

Exemption from calling at Canadian Ports.-If sufficient cargo is not forthcoming from any of the ports of call in Canada, the minister may relieve the contractors from the obligation of calling at such ports.

\section{DISTANCES.}

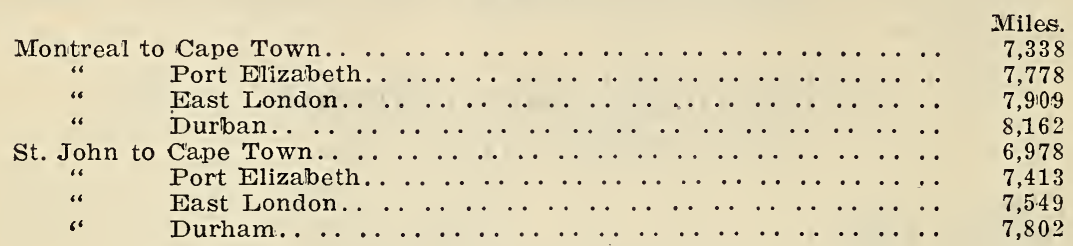

DESCRIPTION OF VESSELS EMPLOYED.

\begin{tabular}{|c|c|c|c|c|c|c|c|c|c|c|c|c|c|c|}
\hline \multirow[b]{2}{*}{ Name. } & \multicolumn{3}{|c|}{ Dimensions. } & \multicolumn{3}{|c|}{ Tonnage. } & \multirow{2}{*}{ 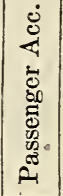 } & \multirow{2}{*}{ 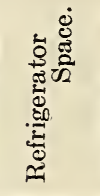 } & \multirow[b]{2}{*}{$\begin{array}{l}\dot{H} \\
\dot{z} \\
\dot{z}\end{array}$} & \multirow[b]{2}{*}{ 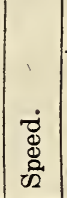 } & \multicolumn{2}{|c|}{ Built. } & \multirow[b]{2}{*}{ In } & \multirow[b]{2}{*}{ Of } \\
\hline & 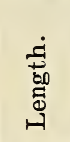 & 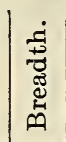 & 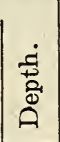 & 苍 & $\begin{array}{l}\dot{0} \\
0 \\
0 \\
0 \\
0 \\
0\end{array}$ & 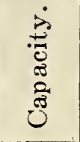 & & & & & At & & & \\
\hline Kaduna & Ft. & $\begin{array}{c}\mathrm{Ft} . \\
52 \cdot 0\end{array}$ & $\begin{array}{l}\text { Ft. } \\
26 \cdot 2\end{array}$ & 2,308 & 4,455 & 8,100 & 12 & $\begin{array}{l}\text { Cu. ft. } \\
10,000\end{array}$ & 339 & $\begin{array}{c}\mathrm{Kts} \\
10\end{array}$ & Middlesbro. & & 1910 & Steel. \\
\hline Kwarr & $360 \cdot \mathrm{c}$ & $052 \cdot 0$ & $26 \cdot 1$ & 2,304 & 4,441 & 8,100 & 12 & $10 \cdot 000$ & 428 & 10 & Middlesbro.. & & 1910 & Steel. \\
\hline Benguela & $425 \cdot$ & $553 \cdot 0$ & $29 \cdot 2$ & 3,534 & 5,520 & 8,920 & 4 & 10,390 & 556 & 12 & Newcastle-on & & 1910 & Steel. \\
\hline New Georgia. & $412 \cdot t$ & $655 \cdot 8$ & $34 \cdot 4$ & 4,044 & 6,566 & 10,660 & Nil. & Nil. & 517 & 11 & Belfast...... & & $19: 8$ & Stee \\
\hline New Mexico.. & $412 \cdot$ & $655 \cdot 8$ & $34 \cdot 4$ & 4,044 & 6,566 & 10,660 & Nil. & Nil. & 517 & 11 & Belfast. & & 1919 & Stee \\
\hline New Brighton & 41 & $655 \cdot 8$ & $34 \cdot 4$ & 4,023 & 6,538 & 10,550 & Nil. & Nil. & & 11 & Belfast. & & 1920 & Ste \\
\hline Jekri......... & 385 & $351 \cdot 2$ & $25 \cdot 5$ & 4,278 & 5,875 & 9,020 & Nil. & Nil. & 440 & 11 & Japan.. & & 1917 & Stee \\
\hline NewBrooklyn & 412 & $655 \cdot 8$ & $34 \cdot 4$ & 4,044 & 6,566 & 10,600 & Nil. & Nil. & 517 & 71 & Belfast. & & 1920 & Stee \\
\hline Fantee....... & 399 & $553 \cdot 0$ & $32 \cdot 8$ & 3,527 & 5,663 & 9,000 & Nil. & Nil. & 517 & 7 & Newcastle & & 1920 & Stee \\
\hline Bereby. & $400 \cdot 2$ & $252 \cdot 3$ & & 3,197 & 5,248 & 8,130 & Nil. & Nil. & 369 & 11 & Hartlepool. & & 1919 & Steel. \\
\hline
\end{tabular}

TRAFFIC RETURNS (Outward voyages).

No cargo is carried inward.

\begin{tabular}{|c|c|c|c|c|c|c|c|}
\hline \multirow[t]{2}{*}{$\begin{array}{l}\text { Calendar } \\
\text { Year. }\end{array}$} & \multirow[t]{2}{*}{$\begin{array}{l}\text { No. of } \\
\text { Trips run. }\end{array}$} & $\begin{array}{l}\text { Number } \\
\text { of Passengers } \\
\text { Carried. }\end{array}$ & \multicolumn{2}{|c|}{$\begin{array}{c}\text { Tons of } \\
\text { Freight Carried. }\end{array}$} & \multirow[t]{2}{*}{ Live Stock. } & \multirow[t]{2}{*}{ Mails. } & \multirow[t]{2}{*}{ Subsidy Paid. } \\
\hline & & & Weight. & Meas. & & & \\
\hline $\begin{array}{l}1912 \ldots \ldots \ldots \\
1913 \ldots \ldots \ldots \\
1914 \ldots \ldots \ldots \\
1915 \ldots \ldots \ldots \\
1916 \ldots \ldots \ldots \\
1917 \ldots \ldots \ldots \\
1918 \ldots \ldots \ldots \\
1919 \ldots \ldots \ldots \\
1920 \ldots \ldots \ldots\end{array}$ & $\begin{array}{r}12 \\
14 \\
12 \\
12 \\
10 \\
9 \\
3 \\
11 \\
12\end{array}$ & 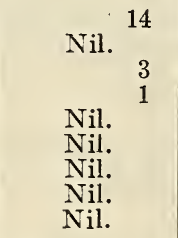 & $\begin{array}{r}31,210 \\
45,316 \\
45,296 \\
52,543 \\
30,797 \\
23,140 \\
9,972 \\
22,503 \\
35,956\end{array}$ & $\begin{array}{r}34,980 \\
53,498 \\
33,563 \\
37,166 \\
38,630 \\
36,653 \\
9,054 \\
49,038 \\
56,970\end{array}$ & 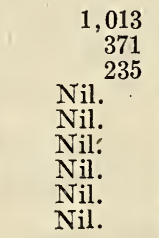 & $\begin{array}{l}\text { Nil. } \\
\text { Nil. } \\
\text { Nil. } \\
\text { Nil. } \\
\text { Nil. } \\
\text { Nil. } \\
\text { Nil. } \\
\text { Nil. } \\
\text { Nil. }\end{array}$ & 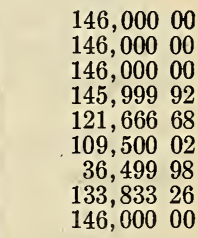 \\
\hline
\end{tabular}


SESSIONAL PAPER NO. 10a

ORIGIN, QUANTITY AND VALUE OF CARGO EXPORTED FROM CANADA

\begin{tabular}{|c|c|c|c|c|c|c|c|c|c|}
\hline \multirow{2}{*}{$\begin{array}{c}\text { Calendar } \\
\text { Year. }\end{array}$} & \multicolumn{3}{|c|}{ Canadian Origin. } & \multicolumn{3}{|c|}{ United States Origin. } & \multicolumn{3}{|c|}{ Total. } \\
\hline & $\begin{array}{c}\text { Tons } \\
\text { Weight. }\end{array}$ & $\begin{array}{c}\text { Tons } \\
\text { Measure- } \\
\text { ment. }\end{array}$ & Value. & $\begin{array}{c}\text { Tons } \\
\text { Weight. }\end{array}$ & $\mid \begin{array}{c}\text { Tons } \\
\text { Measure- } \\
\text { ment. }\end{array}$ & Value. & $\begin{array}{c}\text { Tons } \\
\text { Weight. }\end{array}$ & $\begin{array}{c}\text { Tons } \\
\text { Measure- } \\
\text { ment. }\end{array}$ & Value. \\
\hline $\begin{array}{l}912 \ldots \ldots \ldots \\
913 \ldots \ldots \ldots \\
914 \ldots \ldots \ldots \\
915 \ldots \ldots \ldots \\
916 \ldots \ldots \ldots \\
917 \ldots \ldots \ldots \\
918 \ldots \ldots \ldots \\
919 \ldots \ldots \ldots \\
920 \ldots \ldots \ldots\end{array}$ & $\begin{array}{r}28,901 \\
43,128 \\
42,741 \\
46,981 \\
28,465 \\
20,404 \\
9,889 \\
22,165 \\
34,387\end{array}$ & $\begin{array}{r}22,090 \\
31,196 \\
22,488 \\
23,589 \\
24,600 \\
24,209 \\
8,630 \\
36,509 \\
34,259\end{array}$ & $\begin{array}{c}\$ \\
2,856,419 \\
3,791,039 \\
3,236,733 \\
3,889,139 \\
3,350,296 \\
3,644,333 \\
2,714,870 \\
8,348,508 \\
8,274,449\end{array}$ & $\begin{array}{r}2,309 \\
2,188 \\
2,555 \\
5,562 \\
2,332 \\
2,736 \\
83 \\
338 \\
1,569\end{array}$ & $\begin{array}{r}12,890 \\
22,302 \\
11,075 \\
13,577 \\
14,030 \\
12,444 \\
424 \\
12,529 \\
22,711\end{array}$ & $\begin{array}{r}\$ \\
1,257,263 \\
2,078,630 \\
948,339 \\
1,331,441 \\
1,430,772 \\
1,390,856 \\
74,670 \\
2,237,072 \\
3,192,026\end{array}$ & $\begin{array}{r}31,210 \\
45,316 \\
45,296 \\
52,543 \\
30,797 \\
23,140 \\
9,972 \\
22,503 \\
35,956\end{array}$ & $\begin{array}{r}34,980 \\
53,498 \\
33,563 \\
37,166 \\
38,630 \\
36,653 \\
9,054 \\
49,038 \\
56,970\end{array}$ & $\begin{array}{c}\$ \\
4,113,682 \\
5,869,669 \\
4,185,072 \\
5,220,580 \\
4,781,068 \\
5,035,189 \\
2,789,540 \\
10,585,580 \\
11,466,475\end{array}$ \\
\hline
\end{tabular}

\section{PRINCIPAL ARTICLES EXPORTED}

Of Canadian Origin.-Agricultural implements, calcium carbide, automobiles, paper, lumber, cereal foods, eggfillers, chairs, woodenware, cotton duck, Beaver board, nails, iron and steel, malt, horseshoes, condensed milk, locomotives, cardboard, shovels, spades and cement.

Of United States Origin.-Automobiles, mining machinery, fruit jars, canned meats, washing powder, ammonia, agricultural implements and tractors. 



\title{
PACIFIC OCEAN SERVICES
}

\author{
CANADA AND AUSTRALIA OR NEW ZEALAND \\ Union Steamship Company of New Zealand, Ltd.
}

Contract No. 27.

T. \& C. File No. 25921.

Vote 158.-Canada and Austratia or New Zealand, or both, on the Pacific Ocean, steam service between-

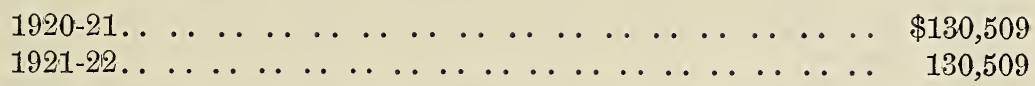

Contractors.-The Union Steamship Co., of New Zealand, Ltd. (Canadian address: Canadian Australasian Royal Mail Line, 440 Seymour street, Vancouver, B.C.)

Contract Dated.-July 13, 1920.

Duration of Contract.-August 1, 1920, to July 31, 1921.

Service.-Sailing approximately every four weeks.

Ports of Call.-Vancouver, B.C.; Victoria, B.C.; Honolulu, in the Sandwich islands; Suva; in the Fiji islands; and Auckland, N.Z.

At the contractor's option, each voyage from Canada to New Zealand may be extended to a port or ports in Australia. The Australian port of call is Sydney.

The call at Suva is conditional upon the Government of Fiji also continuing their contract for a like period and on the same terms as heretofore, and also upon their maintaining the same charges for light and other dues levied on ships employed in the service.

Speed required.-Duration of voyage is not to exceed 20 days, including one day's detention at Honolulu.

Subsidy.—£26,816.18.4 per annum.

Provided that the contractors shall be entitled to receive such subsidy as the Government of New Zealand and Fiji may pay towards the service; and also the Government of Australia, should the service be extended to that Commonwealth.

Deductions from Subsidy.- $\$ 30$ are to be deducted from the amount of subsidv payable on each claim for every complete period of twenty-four hours by which the time occupied in conveyance of the mails between Auckland and Vancouver has exceeded twenty days.

Preference to Canadian Shippers.-No discrimination as regards freight or passenger rates is to be made against Canadian ports, railways, merchants or shippers. Canadian merchants and shippers are to have preference at all times for the carriage of their goods over other merchants and shippers, as far as regards the Canadian connection.

$10 \mathrm{a}-2$ 
11 GEORGE V, A. 1921

Freight and Passenger Rates.-Freight rates from Vancouver or Victoria to New Zealand shall not exceed the current rates charged on similar cargo to New Zealand ports by Union S.S. Co. of N.Z. Limited Mail Steamers from San Francisco.

Passenger rates from Vancouver or Victoria to Auckland shall not exceed passenger rates during the same period from Auckland to Victoria or Vancouver, and return fares from Canadian ports to New Zealand ports shall not exceed return fares in the opposite direction during the same period.

Mails.-To be carried free.

Canadian Trade Commissioners.-To be carried free.

DISTANCES

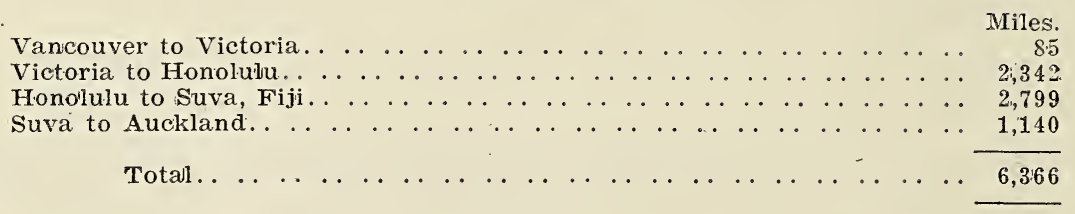

DESCRIPTION OF VESSELS EMPLOYED

\begin{tabular}{|c|c|c|c|c|c|c|c|c|c|c|c|c|c|c|c|}
\hline \multirow[b]{2}{*}{ Name. } & \multicolumn{3}{|c|}{ Dimensions. } & \multicolumn{3}{|c|}{ Tonnage. } & \multicolumn{3}{|c|}{$\begin{array}{l}\text { Passenger } \\
\text { Accommo- } \\
\text { dation. }\end{array}$} & \multirow{2}{*}{ 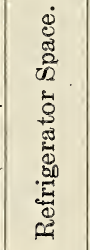 } & \multirow[b]{2}{*}{$\begin{array}{l}\dot{2} \\
\dot{z} \\
z\end{array}$} & \multirow[b]{2}{*}{$\begin{array}{l}\dot{d} \\
\dot{0} \\
\dot{0} \\
\text { की }\end{array}$} & \multicolumn{3}{|c|}{ Built. } \\
\hline & 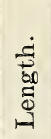 & 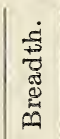 & 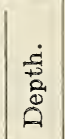 & $\begin{array}{l}\stackrel{+}{0} \\
\ddot{z}\end{array}$ & $\begin{array}{l}\dot{0} \\
\stackrel{0}{0} \\
\stackrel{0}{0} \\
\dot{U}\end{array}$ & 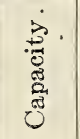 & 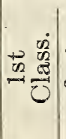 & 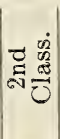 & 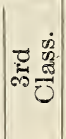 & & & & At & In & Of \\
\hline $\begin{array}{l}\text { Makura... } \\
\text { Niagara... } \\
\text { Tahiti... }\end{array}$ & $\begin{array}{l}\mathrm{Ft} . \\
480 \\
542 \\
460\end{array}$ & $\begin{array}{l}\mathrm{Ft} . \\
58 \\
66 \\
55 \cdot 5\end{array}$ & $\begin{array}{l}\mathrm{Ft} . \\
35 \\
37 \cdot 6 \\
36\end{array}$ & $\begin{array}{l}4,920 \\
7,581 \\
3,841\end{array}$ & $\begin{array}{r}8,200 \\
13,444 \\
7,585\end{array}$ & $\begin{array}{l}3,000 \\
3,800 \\
3,500\end{array}$ & $\begin{array}{l}270 \\
289 \\
160\end{array}$ & $\begin{array}{r}114 \\
210 \\
46\end{array}$ & $\begin{array}{r}72 \\
276 \\
76\end{array}$ & $\begin{array}{l}\mathrm{Cu} . \mathrm{ft} . \\
14,985 \\
63,200 \\
74,651\end{array}$ & $\begin{array}{r}2,035 \\
* 12,500 \\
1,452\end{array}$ & $\begin{array}{l}16 \frac{x}{2} \\
17 \frac{3}{4} \\
16 \frac{1}{2}\end{array}$ & $\begin{array}{l}\text { Glasgow... } \\
\text { Clydebank } \\
\text { Glasgow... }\end{array}$ & $\begin{array}{l}1908 \\
1913 \\
1904\end{array}$ & $\begin{array}{l}\text { Steel } \\
\text { Steel } \\
\text { Steel }\end{array}$ \\
\hline
\end{tabular}

*Indicated Horse Power.

TRAFFIC RETURNS

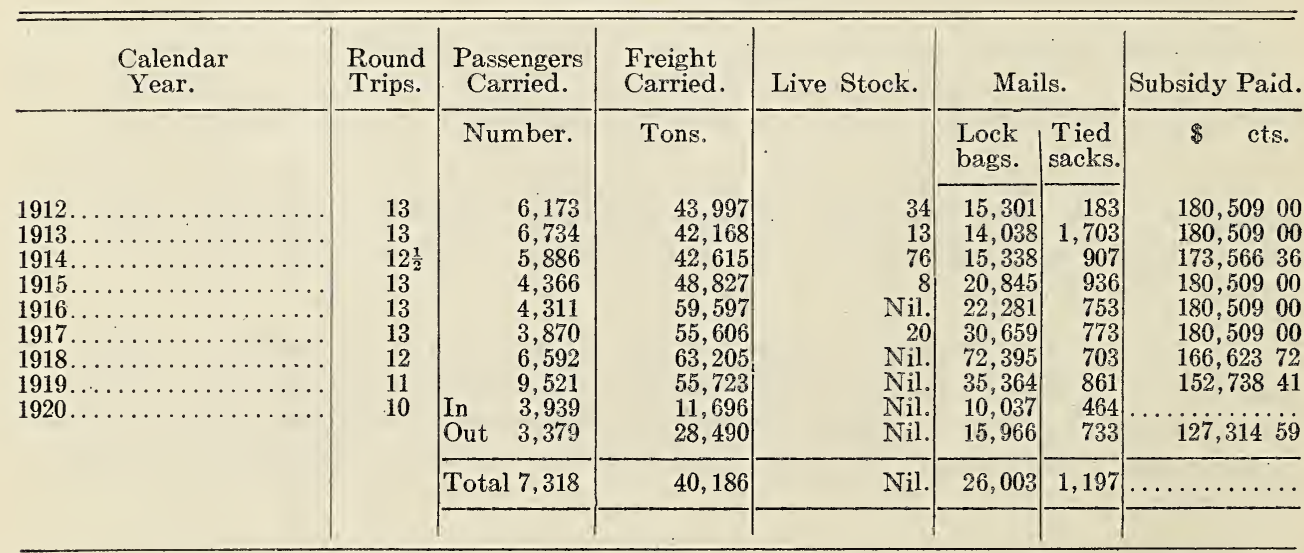

Noте:-One trip was performed by the "Niagara," commencing Nov. 17, 1920, for which traffic returns are not yet available. 
SESSIONAL PAPER No. $10 a$

ORIGIN, QUANTITY AND VALUE OF FREIGHT EXPORTED FROM CANADA

\begin{tabular}{|c|c|c|c|c|c|c|c|c|c|c|}
\hline & \multirow[b]{2}{*}{ To } & \multicolumn{3}{|c|}{ Canadian Origin. } & \multicolumn{3}{|c|}{ United States Origin. } & \multicolumn{3}{|c|}{ Total. } \\
\hline & & $\begin{array}{c}\text { Tons } \\
\text { Weight. }\end{array}$ & $\begin{array}{c}\text { Tons } \\
\text { Measure- } \\
\text { ment. }\end{array}$ & Value. & $\begin{array}{c}\text { Tons } \\
\text { Weight. }\end{array}$ & $\left|\begin{array}{c}\text { Tons } \\
\text { Measure- } \\
\text { ment. }\end{array}\right|$ & Value. & $\begin{array}{c}\text { Tons } \\
\text { Weight. }\end{array}$ & $\begin{array}{c}\text { Tons } \\
\text { Measure- } \\
\text { ment. }\end{array}$ & Value. \\
\hline 1912 & $\begin{array}{l}\text { Auckland..... } \\
\text { Suva......... } \\
\text { Honolulu...... } \\
\text { Sydney...... }\end{array}$ & $\begin{array}{r}1,599 \\
2,604 \\
203 \\
4,960\end{array}$ & $\begin{array}{l}\text { Nil. } \\
\text { Nil. } \\
\text { Nil. } \\
\text { Nil. }\end{array}$ & $\begin{array}{r}\$ \\
263,581 \\
115,075 \\
26,654 \\
501,731\end{array}$ & $\begin{array}{c}1,136 \\
237 \\
\text { Nil. } \\
3,991\end{array}$ & $\begin{array}{l}\text { Nil. } \\
\text { Nil. } \\
\text { Nil. } \\
\text { Nil. }\end{array}$ & \begin{tabular}{|c|}
$\$$ \\
535,463 \\
50,858 \\
Nil. \\
$1,460,089$
\end{tabular} & $\begin{array}{r}2,833 \\
2,841 \\
203 \\
8,952\end{array}$ & $\begin{array}{l}\text { Nil. } \\
\text { Nil. } \\
\text { Nil. } \\
\text { Nil. }\end{array}$ & $\begin{array}{r}\$ \\
799,044 \\
165,933 \\
26,654 \\
1,961,820\end{array}$ \\
\hline & Total. & 9,366 & Nil. & 907,041 & 5,364 & Nil. & $2,046,410$ & 14,829 & Nil. & $2,953,451$ \\
\hline 1913 & $\begin{array}{l}\text { Auckland.. } \\
\text { Suva....... } \\
\text { Honolutu... } \\
\text { Sydney... }\end{array}$ & $\begin{array}{l}200 \\
113 \\
175 \\
530\end{array}$ & $\begin{array}{r}3,167 \\
3,646 \\
40 \\
5,699\end{array}$ & $\begin{array}{r}245,877 \\
121,949 \\
25,977 \\
383,609\end{array}$ & $\begin{array}{r}1,206 \\
106 \\
1 \\
3,260\end{array}$ & $\begin{array}{r}964 \\
142 \\
296 \\
2,263\end{array}$ & $\begin{array}{r}629,541 \\
41,240 \\
337 \\
1,681,011 \\
\end{array}$ & $\begin{array}{r}1,406 \\
219 \\
176 \\
3,790\end{array}$ & $\begin{array}{r}4,131 \\
3,788 \\
40 \\
7,962\end{array}$ & $\begin{array}{r}875,418 \\
163,189 \\
26,314 \\
2,064,620\end{array}$ \\
\hline & Total & 1,018 & 12,552 & 777,412 & 4,573 & 3,369 & $2,352,129$ & 5,591 & 15,921 & $3,129,541$ \\
\hline 1914 & $\begin{array}{l}\text { Auckland.. } \\
\text { Suva....... } \\
\text { Honolulu... } \\
\text { Sydney ... }\end{array}$ & $\begin{array}{r}967 \\
138 \\
136 \\
2,377 \\
\end{array}$ & $\begin{array}{r}2,434 \\
3,901 \\
36 \\
8,737 \\
\end{array}$ & $\begin{array}{r}285,999 \\
128,844 \\
25,748 \\
585,927 \\
\end{array}$ & $\begin{array}{r}861 \\
87 \\
\text { Nil. } \\
2,916 \\
\end{array}$ & $\begin{array}{r}1,054 \\
160 \\
\text { Nil. } \\
1,421 \\
\end{array}$ & $\begin{array}{r}535,646 \\
42,276 \\
\text { Nil. } \\
1,595,831 \\
\end{array}$ & $\begin{array}{r}1,828 \\
225 \\
136 \\
5,293 \\
\end{array}$ & $\begin{array}{r}3,488 \\
4,061 \\
36 \\
10,158 \\
\end{array}$ & $\begin{array}{r}831,645 \\
171,120 \\
25,748 \\
2,181,758 \\
\end{array}$ \\
\hline & Total. . & 3,618 & 15,108 & $1,026,518$ & 3,864 & 2,635 & $2,1 \% 3,753$ & 7,482 & 17,743 & $3,200,271$ \\
\hline 1915 & $\begin{array}{l}\text { Auckland.. } \\
\text { Suva....... } \\
\text { Honolulu... } \\
\text { Sydney.... }\end{array}$ & $\begin{array}{r}4,482 \\
1,045 \\
2 \\
2,765\end{array}$ & $\begin{array}{r}2,450 \\
2,861 \\
2 \\
5,700\end{array}$ & $\begin{array}{r}509,051 \\
176,329 \\
923 \\
927,085\end{array}$ & $\begin{array}{r}614 \\
142 \\
-\quad 722\end{array}$ & $\begin{array}{r}1,356 \\
217 \\
-\quad 500\end{array}$ & $\begin{array}{r}433,959 \\
44,213 \\
- \\
1,483,945 \\
\end{array}$ & $\begin{array}{r}5,096 \\
1,187 \\
2 \\
4,487\end{array}$ & $\begin{array}{r}3,806 \\
3,078 \\
2 \\
10,200\end{array}$ & $\begin{array}{r}942,990 \\
220,542 \\
923 \\
2,411,030\end{array}$ \\
\hline & Total. & 8,294 & 110,513 & $1,613,388$ & 2,478 & 6,073 & $1,962,097$ & 10,772 & 17,086 & $3,575,485$ \\
\hline 1916 & $\begin{array}{l}\text { Auckland... } \\
\text { Suva....... } \\
\text { Honolulu... } \\
\text { Sydney... }\end{array}$ & $\begin{array}{r}3,084 \\
910 \\
1 \\
3,525 \\
\end{array}$ & $\begin{array}{r}4,604 \\
1,850 \\
36 \\
9,533\end{array}$ & $\begin{array}{r}645,970 \\
160,285 \\
3,602 \\
1,380,367\end{array}$ & $\begin{array}{r}409 \\
-\quad 79 \\
-\quad 580\end{array}$ & $\begin{array}{r}3,405 \\
687 \\
-\quad 8 \\
8,843\end{array}$ & $\begin{array}{r}511,380 \\
88,275 \\
\overline{1}, \overline{07}, 810 \\
\end{array}$ & $\begin{array}{r}3,493 \\
989 \\
1 \\
4,105\end{array}$ & $\begin{array}{r}8, v 09 \\
2,537 \\
36 \\
18,376\end{array}$ & $\begin{array}{r}1,157,3: 0 \\
248,560 \\
3,6 C 2 \\
3,088,207\end{array}$ \\
\hline & Total. & 7,520 & 16,023 & $2,190,224$ & 1,068 & 12,935 & $2,307,495$ & 8,588 & 28,958 & $4,497,719$ \\
\hline 1917 & $\begin{array}{l}\text { Auckland. . } \\
\text { Suva....... } \\
\text { Honolulu... } \\
\text { Sydney.... }\end{array}$ & $\begin{array}{r}4,813 \\
54 \\
-\quad \\
2,809\end{array}$ & $\begin{array}{r}6,211 \\
1,353 \\
38 \\
8,419\end{array}$ & $\begin{array}{r}1,099,650 \\
108,806 \\
3,527 \\
1,301,674\end{array}$ & $\begin{array}{r}77 \\
-\quad 1 \\
-\quad 1\end{array}$ & $\begin{array}{r}2,696 \\
396 \\
-\quad 0 \\
5,091\end{array}$ & $\begin{array}{r}411,012 \\
64,233 \\
\overline{1}, 121,660\end{array}$ & $\begin{array}{r}4,890 \\
55 \\
-\quad \\
2,809\end{array}$ & $\begin{array}{r}8,907 \\
1,749 \\
38 \\
13,510\end{array}$ & $\begin{array}{r}1,510,662 \\
173,039 \\
3,527 \\
2,423,334\end{array}$ \\
\hline & Total.. & 7,676 & 16,021 & $2,513,657$ & 78 & 8,183 & $1,596,905$ & 7,754 & 24,204 & $4,110,562$ \\
\hline 1918 & $\begin{array}{l}\text { Auckland.. } \\
\text { Suva....... } \\
\text { Honolulu... } \\
\text { Sydney... }\end{array}$ & $\begin{array}{l}566 \\
- \\
775\end{array}$ & $\begin{array}{r}20,400 \\
1,457 \\
105 \\
5,593\end{array}$ & $\begin{array}{r}2,038,144 \\
225,363 \\
8,991 \\
1,641,174\end{array}$ & $\begin{array}{r}58 \\
9 \\
-\quad 72\end{array}$ & $\begin{array}{r}2,448 \\
212 \\
-\quad 3,367\end{array}$ & $\begin{array}{r}850,256 \\
68,792 \\
\overline{\overline{6}}, 129\end{array}$ & $\begin{array}{r}624 \\
-\quad 9 \\
847\end{array}$ & $\begin{array}{r}22,848 \\
1,669 \\
105 \\
9,960\end{array}$ & $\begin{array}{r}2,888,400 \\
294,155 \\
8,991 \\
3,337,303\end{array}$ \\
\hline & Total... & $1,34: 1$ & 27,555 & $3,913,672$ & 139 & 7,027 & $2,615,177$ & 1,480 & 34,582 & $6,528,849$ \\
\hline 1919 & $\begin{array}{l}\text { Auckland.. } \\
\text { Suva........ } \\
\text { Honolulu... } \\
\text { Sydney.... }\end{array}$ & $\begin{array}{r}284 \\
\cdots \\
110\end{array}$ & $\begin{array}{r}21,922 \\
838 \\
45 \\
4,619 \\
\end{array}$ & $\begin{array}{r}2,161,146 \\
141,296 \\
6,700 \\
1,645,625 \\
\end{array}$ & $\begin{array}{r}58 \\
7 \\
192 \\
\end{array}$ & $\begin{array}{r}2,030 \\
226 \\
11 \\
3,869 \\
\end{array}$ & $\begin{array}{r}710,228 \\
61,950 \\
2,299 \\
1,572,273 \\
\end{array}$ & $\begin{array}{r}342 \\
7 \\
1 \\
302 \\
\end{array}$ & $\begin{array}{r}23,952 \\
1,064 \\
56 \\
8,488 \\
\end{array}$ & $\begin{array}{r}2,871,374 \\
203.246 \\
8,999 \\
3,217,898 \\
\end{array}$ \\
\hline & Total. & 395. & 27,424 & $3,954,767$ & 257 & 6,136 & $2,346,750$ & 652 & 33,560 & $6,301,517$ \\
\hline 1920 & $\begin{array}{l}\text { Auckland. } \\
\text { Suva...... } \\
\text { Honolulu... } \\
\text { Sydney... }\end{array}$ & $\begin{array}{r}2,475 \\
431 \\
21 \\
809\end{array}$ & $\begin{array}{r}14,161 \\
1,262 \\
15 \\
5,277\end{array}$ & $\begin{array}{r}1,895,106 \\
225,136 \\
2,625 \\
1,255,756\end{array}$ & $\begin{array}{r}201 \\
-\quad 3 \\
804\end{array}$ & $\begin{array}{r}2,725 \\
327 \\
18 \\
2,516\end{array}$ & $\begin{array}{r}573,066 \\
69,454 \\
9,319 \\
1,274,937\end{array}$ & $\begin{array}{r}2,676 \\
434 \\
21 \\
1,613\end{array}$ & $\begin{array}{r}16,886 \\
1,589 \\
33 \\
7,793\end{array}$ & $\begin{array}{r}2,468,172 \\
294,590 \\
11,944 \\
2,530,693\end{array}$ \\
\hline & Total. & 3,736 & 20,715 & $3,378,623$ & 1,008 & 5,5 & $1,926,776$ & 4,744 & 26,301 & $5,305,399$ \\
\hline
\end{tabular}

$10 a-2 \frac{1}{2}$ 


\section{PRINCIPAL ARTICLES EXPORTED}

Of Canadian Origin.-Canned salmon, fresh fruit (in cold storage), potatoes, onions, leather and rubber goods, lumber, codfish, whisky, bicycles and parts thereof, chairs, corsets, suspenders, sewing machines, hardware and machinery.

Of United States Origin.-Drugs, sewing machines, automobiles, gas engines and other machinery, soap and scouring powders, leather and rubber goods, cash registers and scales, cereal foods, telephone material, adding machines, vacuum cleaners, motor cycles, and corsets.

\section{PRINCE RUPERT, B.C., AND QUEEN CHARLOTTE ISLANDS}

\section{The Grand Trunt Pacific Coast S. S. Co., Ltd.}

Contract No. 61.

T. \& C. File No. 26129.

Vote 159.-Prince Rupert, B.C., and Queen Charlotte Tslands-steam service between-

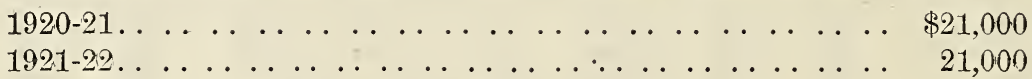

Contractors.-The Grand Trunk Pacific Coast S. S. Co., Ltd.

Date of Contract.-September 23, 1920.

Druation of Contract.-April 1, 1920, to March 31, 1921.

Services and Ports of Call.-Fortnightly trips from Prince Rupert, B.C., calling each way at Refuge Bay (on Porcher Island), and Masset, Port Clements, Sandspit, Skidegate, Queen Charlotte, Jedway, Thurston Harbour, Lockeport, and Atli Inlet, and calling once each month at Cumshewa Inlet, it being understood that the call at Refuge Bay shall be made by a subsidiary launch service provided by the contractors; calling at the option of the company when deemed necessary at Ketchikan, Alaska.

Speed Required.-Not stated. April.

Subsidy.-\$21,000 per annum, payable quarterly in July, October, January and

Mails.-To be carried free.

Government Wharves.-Steamer must call whenerer possible.

\section{DISTANCES.}

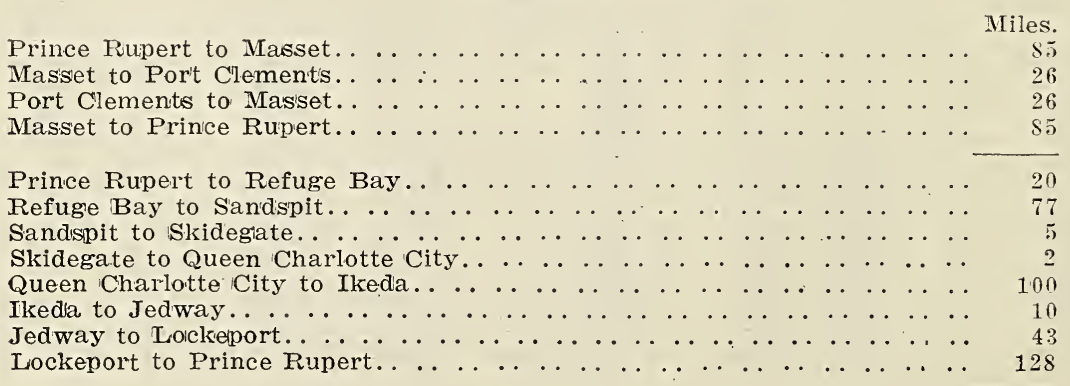


DESCRIPTION OF VESSELS EMPLOYED

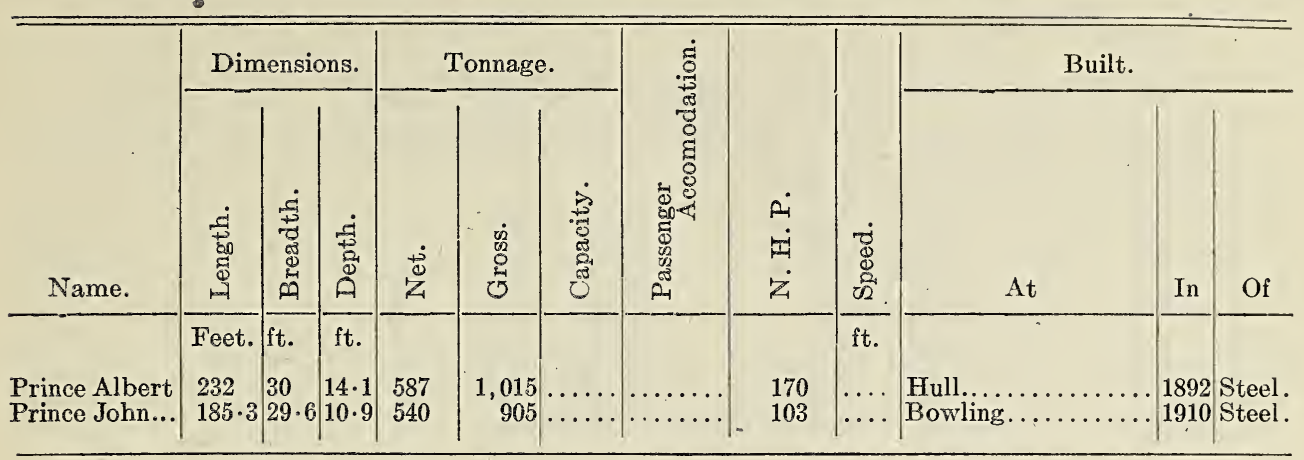

TRAFFIC RETURNS

\begin{tabular}{|c|c|c|c|c|c|c|c|c|}
\hline \multirow{2}{*}{$\begin{array}{l}\text { Calendar } \\
\text { Year. }\end{array}$} & \multirow{2}{*}{$\begin{array}{l}\text { No. of } \\
\text { Round } \\
\text { trips run. }\end{array}$} & \multirow[b]{2}{*}{$\begin{array}{c}\text { Passengers } \\
\text { Carried. }\end{array}$} & \multicolumn{2}{|c|}{ Freight. } & \multirow{2}{*}{$\mid \begin{array}{c}\text { Live } \\
\text { Stock }\end{array}$} & \multicolumn{2}{|c|}{ Mails. } & \multirow{2}{*}{$\begin{array}{l}\text { Subsidy } \\
\text { Paid. }\end{array}$} \\
\hline & & & $\begin{array}{c}\text { tons } \\
\text { Weight. }\end{array}$ & $\mid \begin{array}{c}\text { Tons } \\
\text { Measurement. }\end{array}$ & & $\begin{array}{l}\text { Lock } \\
\text { Bags. }\end{array}$ & $\begin{array}{c}\text { Tied } \\
\text { Sacks }\end{array} \mid$ & \\
\hline 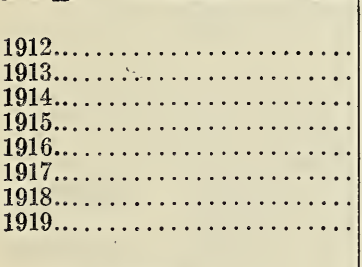 & $\begin{array}{l}44 \\
40 \\
35 \\
13 \\
25 \\
24 \\
34 \\
39\end{array}$ & \begin{tabular}{|r|r}
5,910 \\
4,588 \\
3,175 \\
686 \\
1,584 \\
2,217 \\
11,157 \\
In... .1,562 \\
Out. 1,729
\end{tabular} & $\begin{array}{r}3,250 \\
3,923 \\
2,695 \\
1,380 \\
5,264 \\
6,553 \\
17,395 \\
3,776 \\
2,172\end{array}$ & 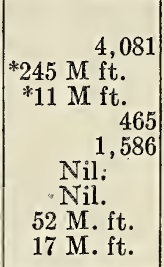 & $\begin{array}{r}60 \\
124 \\
40 \\
117 \\
116 \\
5 \\
35 \\
18 \\
6\end{array}$ & $\begin{array}{r}1,328 \\
2,359 \\
1,780 \\
1,321 \\
2,116 \\
1,951 \\
4,231 \\
994 \\
2,341\end{array}$ & \begin{tabular}{|}
1,957 \\
1,585 \\
1,570 \\
Nil. \\
Nil. \\
209 \\
551 \\
3 \\
30
\end{tabular} & $\begin{array}{rr}\$ & \\
8,200 & 00 \\
8,000 & 00 \\
7,000 & 03 \\
7,333 & 30 \\
6,000 & 00 \\
19,750 & 00 \\
21,000 & 00\end{array}$ \\
\hline Total............. & $\ldots \ldots \ldots \ldots$ & 3,291 & 5,948 & 69 M. ft. & 24. & 3,335 & 33 & 21,00000 \\
\hline $1920 \ldots \ldots \ldots \ldots \ldots \ldots \ldots$ & 38 & $\begin{array}{l}\text { In..... .2,778 } \\
\text { Out. } \quad 2,788\end{array}$ & $\begin{array}{l}3,497 \\
1,661\end{array}$ & $\begin{array}{r}236 \\
24\end{array}$ & $\begin{array}{l}\text { Nil. } \\
\text { Nil. }\end{array}$ & $\begin{array}{r}851 \\
2,395\end{array}$ & $\begin{array}{l}25 \\
76\end{array}$ & 21,00000 \\
\hline Total............ & $\ldots \ldots \ldots$ & 5,566 & 5,158 & 260 & Nil. & 3,246 & 101 & \\
\hline
\end{tabular}

${ }^{*}$ Lumber.

The service for 1915,1916 and the first quarter of 1917 was performed by the Union S.S. Co. of B.C., Ltd.

\section{VICTORIA AND SAN FRANCISCO \\ Pacific Steamship Company}

Contract No. 10.

T. \& C. File 26037.

Vote 160.-Victoria and San Francisco.-Steam service between-

$$
\begin{aligned}
& 1920-21 \ldots \ldots \ldots \ldots \ldots \ldots \ldots \ldots \ldots \\
& 1921-22 \ldots \ldots \ldots \ldots \ldots \ldots \ldots \ldots \ldots \text {. . . . . . . . . . . } 3,000
\end{aligned}
$$

Contractors.-The Pacific Steamship Co. of Seattle, Wash., U.S.A.

(Canadian Agents--R. P. Rithet \& Co., Ltd., 1117 Wharf street, Victoria, B.C.)

Date of contract.-August 23, 1920. 
Duration of contract.-April 1, 1920, to March 31, 1921.

Service.-Weekly.

Ports of Call.-Victoria, B.C., and San Francisco, U.S.A.

Subsidy. $-\$ 3,000$ per annum, payable in quarterly instalments on the first days of July, October, January and April.

Speed Required.-Not stated.

Mails.-To be carried free.

Canadian Trade Commissioners. - To be carried free.

Distance.-Victoria to San Francisco, 750 miles.

DESCRIPTION OF VESSELS EMPLOYED.

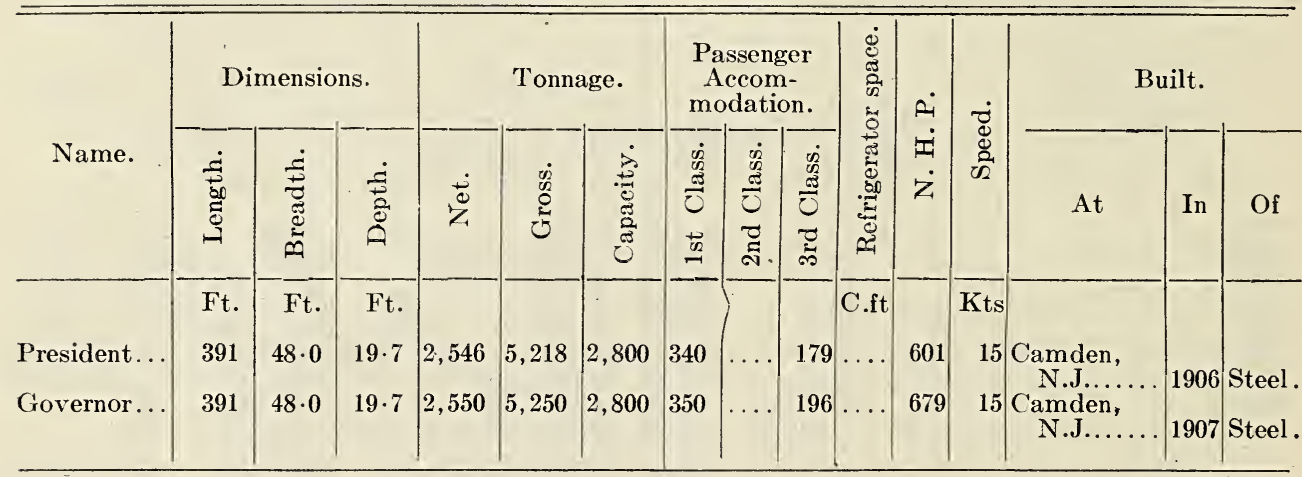

Nore.-A steamship service between Victoria and San Francisco, provided by the Dominion Government, is required under the terms of the agreement by which British Columbia entered Confederation.

TRAFFIC RETURNS.

\begin{tabular}{|c|c|c|c|c|c|c|c|c|}
\hline \multirow{2}{*}{ Calendar Year. } & \multirow{2}{*}{$\begin{array}{c}\text { No. of } \\
\text { Round } \\
\text { Trips run. }\end{array}$} & \multirow{2}{*}{$\begin{array}{c}\text { Number of } \\
\text { Passengers } \\
\text { Carried. }\end{array}$} & \multicolumn{2}{|c|}{$\begin{array}{c}\text { Tons of Freight } \\
\text { Carried. }\end{array}$} & \multirow{2}{*}{$\begin{array}{c}\text { Live } \\
\text { Stock } \\
\text { Carried. }\end{array}$} & \multicolumn{2}{|c|}{$\begin{array}{l}\text { Mails } \\
\text { Carried. }\end{array}$} & \multirow{2}{*}{$\begin{array}{l}\text { Subsidy } \\
\text { Paid. }\end{array}$} \\
\hline & & & Weight. & Meas. & & $\begin{array}{l}\text { Lock } \\
\text { Bags. }\end{array}$ & $\begin{array}{l}\text { Tied } \\
\text { Sacks. }\end{array}$ & \\
\hline $\begin{array}{l}1912 \ldots \\
1913 \ldots \\
1914 \ldots \\
1915 \ldots \\
1916 \ldots \\
1917 \ldots \\
1918 \ldots \\
1919 \ldots\end{array}$ & $\begin{array}{l}52 \\
52 \\
54 \\
52 \\
47 \frac{1}{2} \\
52 \\
43 \frac{1}{2} \\
36\end{array}$ & $\begin{array}{r}2,612 \\
3,791 \\
3,630 \\
4,307 \\
3,249 \\
6,703 \\
3,864 \\
\text { In } 2,286 \\
\text { Out } 2,010\end{array}$ & $\begin{array}{r}6,197 \\
4,779 \\
3,534 \\
1,955 \\
3,702 \\
4,815 \\
2,949 \\
1,933 \\
78\end{array}$ & $\begin{array}{l}8,654 \\
7,306 \\
4,386 \\
2,435 \\
2,514 \\
\text { Nil. } \\
\text { Nil. } \\
\text { Nil. } \\
\text { Nil. }\end{array}$ & $\begin{array}{l}\text { Nil. } \\
\text { Nil. } \\
\text { Nil. } \\
\text { Nil. } \\
\text { Nil. } \\
\text { Nil. } \\
\text { Nil. } \\
\text { Nil. } \\
\text { Nil. }\end{array}$ & $\begin{array}{l}\text { Nil. } \\
\text { Nil. } \\
\text { Nil. } \\
\text { Nil. } \\
\text { Nil. } \\
\text { Nil. } \\
\text { Nil. } \\
\text { Nil. } \\
\text { Nil. }\end{array}$ & $\begin{array}{l}\text { Nil. } \\
\text { Nil. } \\
\text { Nil. } \\
\text { Nil. } \\
\text { Nil. } \\
\text { Nil. } \\
\text { Nil. } \\
\text { Nil. } \\
\text { Nil. }\end{array}$ & \begin{tabular}{ll}
\multicolumn{3}{c}{$\$$} & cts. \\
3,000 & 00 \\
3,000 & 00 \\
2,971 & 15 \\
3,000 & 00 \\
2,769 & 23 \\
3,000 & 00 \\
2,509 & 77 \\
2,076 & 95
\end{tabular} \\
\hline \multirow[t]{3}{*}{ H } & Total.... & 4,296 & 2,011 & Nil. & Nil. & Nil. & Nil. & \multirow{3}{*}{$2,826 \quad 90$} \\
\hline & $49 \frac{1}{2}$ & $\begin{array}{l}\text { In } 2,360 \\
\text { Out } 2,855\end{array}$ & $\begin{array}{r}3,601 \\
243\end{array}$ & $\begin{array}{l}\text { Nil. } \\
\text { Nil. }\end{array}$ & $\begin{array}{l}\text { Nil. } \\
\text { Nil. }\end{array}$ & $\begin{array}{l}\text { Nil. } \\
\text { Nil. }\end{array}$ & $\begin{array}{l}\text { Nil. } \\
\text { Nil. }\end{array}$ & \\
\hline & Total.... & 5,215 & 3,844 & Nil. & Nil. & Nil. & Nil. & \\
\hline
\end{tabular}


SESSIONAL PAPER No. 10a

ORIGIN, QUANTITY AND VALUE OF CARGO EXPORTED FROM CANADA.

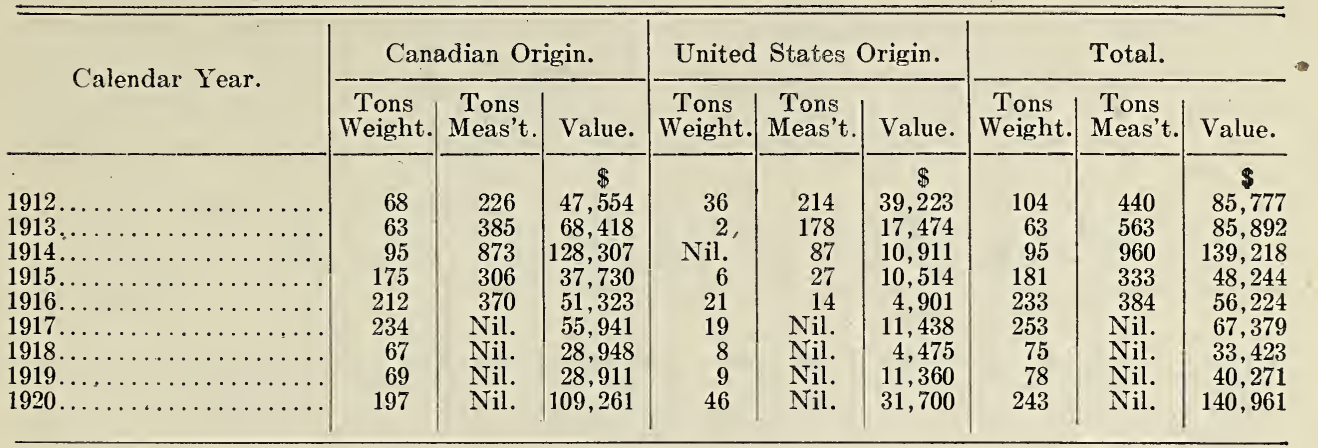

PRINCIPAL ARTICLES EXPORTED

Of Canadian Origin.-Household goods, automobiles, canned clams, paint, woodpulp and beer.

Of Cnited States Origin.-Empty cylinders, automobiles, machinery, raisins and furs.

\section{VICTORIA, VANCOUVER, WAYPORTS AND SKAGWAY}

\section{Canadian Pacific Rallway Company}

Contract No. 28.

T. \& C. File No. 25590.

Vote 161.-Victoria, Vancouver, Wayports and Sliagway, steam service between-

$$
\begin{aligned}
& 1920-21 \ldots \ldots \ldots \ldots \ldots \ldots \text {. . . . . . . . . . . . . . \$25,000 }
\end{aligned}
$$

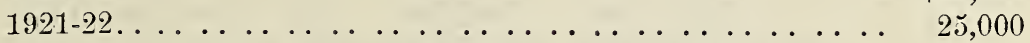

Contractor.-Canadian Pacific Railway Co., Montreal, Que.

Contract Dated.-June 16, 1920.

Duration of Contract.-April 1, 1920, to March 31, 1921.

Service.-Four complete round trips each month from June to October, inclusive; three complete round trips each month from March to May, inclusive; and two complete round trips each month from November to February, inclusive. Skagway.

Ports of Call.-Victoria, Vancouver, Prince Rupert, Ketchikan, Juneau and

Calls at United States Ports.-Steamers are permitted to call at the United States ports mentioned in the preceding paragraph on outward trips only. April.

Subsidy. $-\$ 25,000$ per annum, payable quarterly in July, October, January and

Mails.-To be carried free.

\section{DISTANCEIS.}

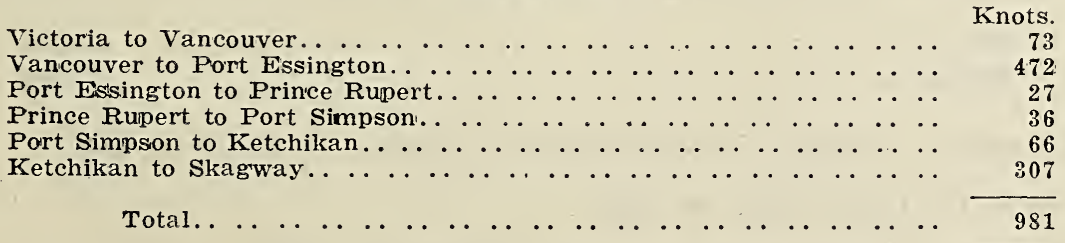


DESCRIPTION OF VESSELS EMPLOYED.

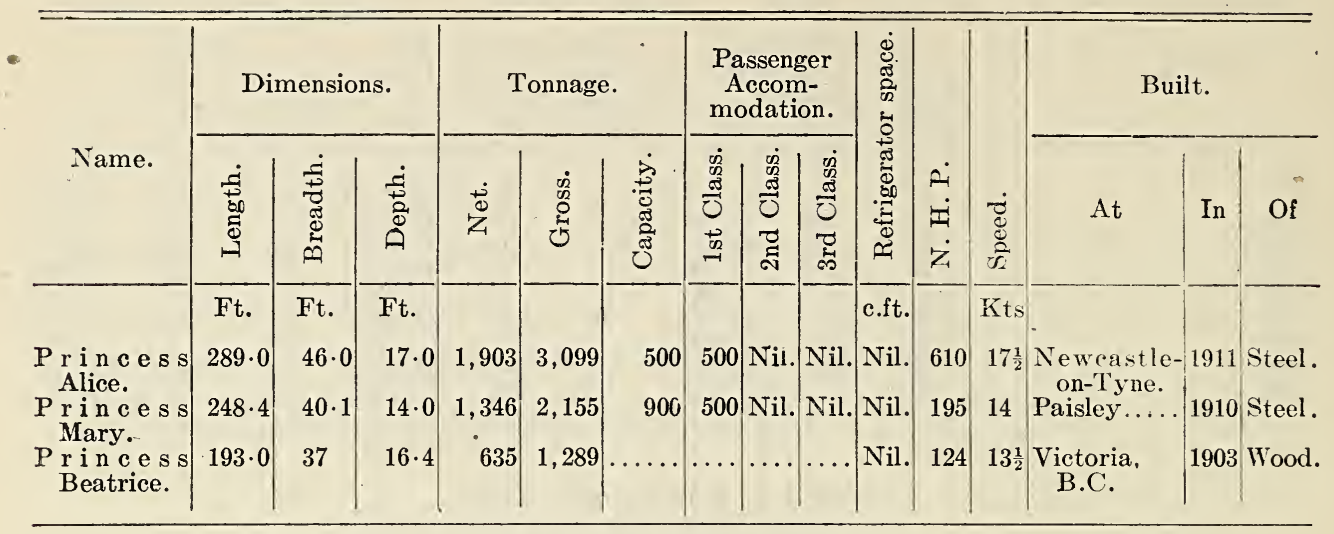

TRAFFIC RETURNYS.

\begin{tabular}{|c|c|c|c|c|c|c|c|c|c|c|}
\hline \multirow[t]{2}{*}{ Calendar Year. } & \multirow[t]{2}{*}{$\begin{array}{c}\text { No. of } \\
\text { Round } \\
\text { Trips run. }\end{array}$} & \multicolumn{3}{|c|}{$\begin{array}{l}\text { Number } \\
\text { of Passengers } \\
\text { Carried. }\end{array}$} & \multicolumn{2}{|c|}{$\begin{array}{l}\text { Tons } \\
\text { of Freight } \\
\text { Carried. }\end{array}$} & \multirow{3}{*}{\begin{tabular}{|r|}
$\begin{array}{c}\text { Live } \\
\text { Stock. }\end{array}$ \\
\\
900 \\
\end{tabular}} & \multicolumn{2}{|c|}{ Mails. } & \multirow{3}{*}{$\begin{array}{c}\begin{array}{c}\text { Subsidies } \\
\text { Paid. }\end{array} \\
\qquad \begin{array}{c}\$ \text { cts. }^{\text {cts. }} \\
12,500\end{array}\end{array}$} \\
\hline & & $\begin{array}{c}\text { 1st } \\
\text { Class. }\end{array}$ & $\begin{array}{c}2 \mathrm{nd} \\
\text { Class. }\end{array}$ & $\begin{array}{c}\text { 3rd } \\
\text { Class. }\end{array}$ & $\begin{array}{c}\text { Tons } \\
\text { W'ght }\end{array}$ & $\begin{array}{c}\text { Tons } \\
\text { Meas. } \\
\end{array}$ & & $\begin{array}{l}\text { Lock } \\
\text { Bags. }\end{array}$ & \begin{tabular}{|l} 
Tied \\
Sacks
\end{tabular} & \\
\hline 1912. & 39 & 10,948 & 1,216 & 404 & $\overline{5,315}$ & 3,238 & & $\overline{6,227}$ & 5,238 & \\
\hline 1913. & 40 & 12,292 & 1,461 & 584 & 6,409 & 1,372 & 482 & 3,460 & 9,118 & 12,500 \\
\hline 1914. & 40 & 12,788 & 827 & 811 & 11,483 & 349 & 925 & 6,665 & 6,377 & 12,500 \\
\hline 1915. & 40 & 6,020 & 601 & 249 & $\overline{10,818}$ & 597 & $\overline{1,521}$ & $\overline{5,067}$ & 7,703 & 12,500 \\
\hline 1916. & 42 & 7,638 & 440 & 199 & 13,506 & 180 & 1,239 & 3,361 & 14,207 & 12,500 \\
\hline $1917 .$. & 40 & 7,616 & 528 & 322 & $\overline{13,909}$ & Nil. & 779 & 4,240 & 9,347 & 12,500 \\
\hline 1918. & $32 \frac{1}{2}$ & 6,308 & 810 & 112 & 16,890 & Nil. & 158 & 4,496 & 8,195 & 11,26303 \\
\hline 1919. & 34 & 9,430 & 699 & 493 & 8,583 & Nil. & 260 & 6,517 & 7,649 & 21,875 \\
\hline \multirow[t]{2}{*}{1920.} & $\begin{array}{ll}35 & \text { In } \\
& \text { Out }\end{array}$ & $\begin{array}{l}5,761 \\
4,414\end{array}$ & $\begin{array}{l}557 \\
148\end{array}$ & $\begin{array}{l}335 \\
137\end{array}$ & \begin{tabular}{|l|}
3,197 \\
6,385
\end{tabular} & $\begin{array}{l}\text { Nil. } \\
\text { Nil. }\end{array}$ & $\begin{array}{r}1 \\
100\end{array}$ & $\begin{array}{l}1,794 \\
2,350\end{array}$ & $\begin{array}{l}2,270 \\
7,110 \\
\end{array}$ & \multirow[t]{2}{*}{$23,593 \quad 75$} \\
\hline & Total... & 10,175 & 705 & 472 & 9,582 & Nil. & 101 & 4,144 & 9,380 & \\
\hline
\end{tabular}

VICTORIA AND WEST COAST. VANCOUVER ISLAND Canadian Pacific Railway Company

Contract No. 63.

T. \& C. File 25820.

Vote 162.-Victoria and West Coast Vancouver Island.-Steam service between$1920-21 \ldots \ldots \ldots \ldots \ldots \ldots$ $1921-22 \ldots \ldots \ldots \ldots \ldots \ldots \ldots$

Contractors.-Canadian Pacific Railway Company, of Montreal, Que.

Date of Contract.-June 18, 1920.

Duration of Contract.-April 1, 1920, to March 31, 1921. 
SESSIONAL PAPER NO. 10a

Service.-Three complete, round trips each month.

Ports of Call.-Victoria, Port Renfrew, Carmanah, Cla-oose, Bamfield, New Alberni, Uchucklesit, Sechart, Ucluclet, Clayoquot, Tofino, Christie School, Ahousaht, Hesquiot, Nootka, Whaling Station, Kyuquot, Quatsino, and Port Alice; and if sufficient business offers at other intermediate accessible ports.

\section{Speed Required.-Not stated.}

Subsidy. $-\$ 15,000$ per annum, payable quarterly in June, September, December and March.

Mails.-To be carried free.

DISTANCES.

Knots.

Victoria to Port Renfrew . . . . . . . . . . . . . . . . . .

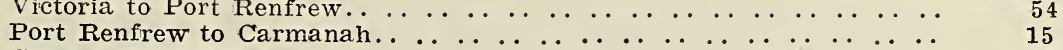

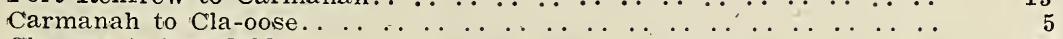

Cla-oose to Bamfield. . . . . . . . . . . $\ldots \ldots \ldots$

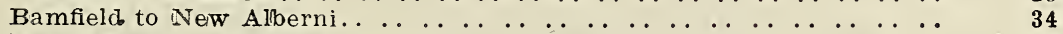

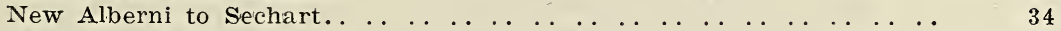

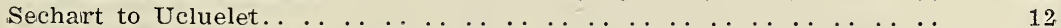

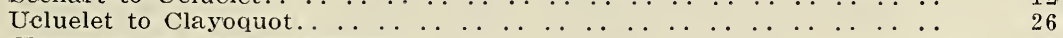

Clayoquot to Christie's School. . . . . . . . . . . . . . . . . . . . . . . . . .

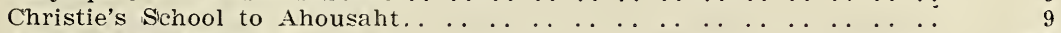

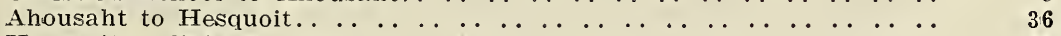

Hesquoit to Friendly Cove. .

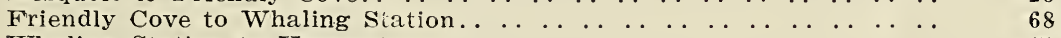

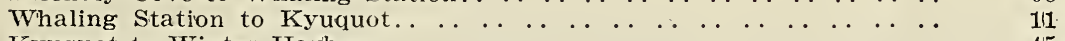

Kyuquot to Winter Harbour. . . . . . . . . . . . . . . . . . . . . . . . .

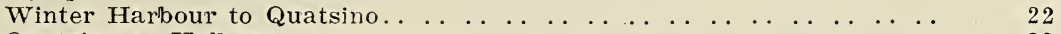

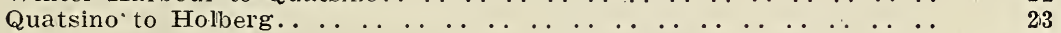

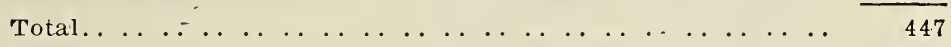

DESCRIPTION OFIVESSELS EMPLOYED.

\begin{tabular}{|c|c|c|c|c|c|c|c|c|c|c|c|c|c|c|c|}
\hline \multirow[b]{2}{*}{ Name. } & \multicolumn{3}{|c|}{ Dimensions. } & \multicolumn{3}{|c|}{ Tonnage. } & \multicolumn{3}{|c|}{$\begin{array}{l}\text { Passenger } \\
\text { Accom- } \\
\text { modation. }\end{array}$} & \multirow{2}{*}{ 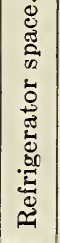 } & \multirow[b]{2}{*}{$\begin{array}{l}\dot{a} \\
\dot{I} \\
\dot{z}\end{array}$} & \multirow[b]{2}{*}{ 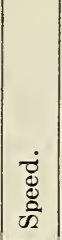 } & \multicolumn{3}{|c|}{ Built. } \\
\hline & 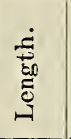 & 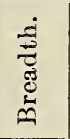 & 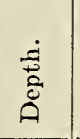 & $\stackrel{\dot{0}}{\dot{0}}$ & $\begin{array}{c}0 \\
0 \\
0 \\
0 \\
0 \\
0\end{array}$ & 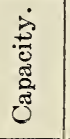 & 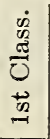 & 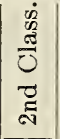 & 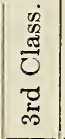 & & & & At & In & Of \\
\hline $\begin{array}{c}\text { Princess } \\
\text { Maquinna. }\end{array}$ & $\begin{array}{l}\text { Ft. } \\
232\end{array}$ & $\begin{array}{r}\text { Ft. } \\
38\end{array}$ & $\begin{array}{r}\text { Ft. } \\
17\end{array}$ & 978 & 1,777 & 800 & 500 & Nil. & Nil. & $\begin{array}{l}\text { C.ft } \\
\text { Nit. }\end{array}$ & Nil. & $\begin{array}{r}\mathrm{Kts} \\
12\end{array}$ & $\begin{array}{l}\text { Victoria, } \\
\text { B.C. }\end{array}$ & 1913 & Steel. \\
\hline
\end{tabular}


TRAFFIC RETURNS.

\begin{tabular}{|c|c|c|c|c|c|c|c|c|c|c|}
\hline \multirow[t]{2}{*}{ Calendar Year. } & \multirow[t]{2}{*}{$\begin{array}{c}\text { No. of } \\
\text { Round } \\
\text { Trips } \\
\text { run. }\end{array}$} & \multicolumn{3}{|c|}{$\begin{array}{l}\text { Passengers } \\
\text { Carriea. }\end{array}$} & \multicolumn{2}{|c|}{$\begin{array}{l}\text { Tons of Freight } \\
\text { Carried. }\end{array}$} & \multirow[t]{2}{*}{$\begin{array}{l}\text { Live } \\
\text { Stock. }\end{array}$} & \multicolumn{2}{|c|}{ Mails. } & \multirow{2}{*}{$\begin{array}{c}\begin{array}{c}\text { Subsidy } \\
\text { Paid. }\end{array} \\
\$ \text { cts. }\end{array}$} \\
\hline & & $\begin{array}{c}1 \text { st } \\
\text { Class. }\end{array}$ & $\begin{array}{l}\text { 2nd } \\
\text { Class. }\end{array}$ & $\begin{array}{c}\text { 3rd } \\
\text { Class }\end{array}$ & $\begin{array}{c}\text { Tons } \\
\text { Weight. }\end{array}$ & $\begin{array}{l}\text { Tons } \\
\text { Meas. }\end{array}$ & & $\begin{array}{l}\text { Lock } \\
\text { Bags. }\end{array}$ & $\begin{array}{c}\text { Tied } \\
\text { Sacks }\end{array}$ & \\
\hline 1912. & 46 & 4,954 & 1,418 & 1,137 & 5,241 & 5,854 & 111 & 1,751 & 937 & 5,00000 \\
\hline 1913. & 43 & 5,173 & 3,534 & Nil. & 1,100 & 7,292 & 73 & 2,057 & 940 & 5,00000 \\
\hline 1914. & 43 & 4,123 & 2,469 & 615 & 6,806 & 1,739 & 62 & 2,479 & 1,101 & 5,00000 \\
\hline 1915 & 42 & 2,984 & 916 & 605 & 7,442 & 5,382 & 40 & 2,936 & 898 & 5,00000 \\
\hline 1916. & 42 & 3,275 & 1,343 & Nil. & 9,756 & Nil. & 20 & 2,321 & 1,817 & 5,00000 \\
\hline $1917 \ldots$ & 42 & 5,292 & 2,169 & Nil. & 11,636 & Nil. & 105 & 4,353 & 174 & 5,00000 \\
\hline 1918. & 36 & 7,168 & $:, 516$ & 438 & 15,930 & Nil. & 151 & 1,302 & 3,936 & $4,374 \quad 94$ \\
\hline 1919. & $36 \frac{1}{2}$ & 5,733 & 1,525 & Nil. & 15,047 & Nil. & 207 & 3,335 & 1,277 & 4,37500 \\
\hline \multirow[t]{2}{*}{$1920 \ldots$} & $\begin{array}{l}36 \text { In } \\
\text { Out }\end{array}$ & $\begin{array}{l}3,428 \\
3,552\end{array}$ & $\begin{array}{l}639 \\
725\end{array}$ & $\begin{array}{l}\text { Nil. } \\
\text { Nil. }\end{array}$ & $\begin{array}{r}14,010 \\
7,040\end{array}$ & $\begin{array}{l}\text { Nil. } \\
\text { Nil. }\end{array}$ & $\begin{array}{r}16 \\
232\end{array}$ & $\begin{array}{r}832 \\
3,147\end{array}$ & $\begin{array}{r}184 \\
1,015\end{array}$ & \multirow[t]{2}{*}{12,50000} \\
\hline & Total. & 6,980 & 1,364 & Nil. & 21,050 & Nil. & 248 & 3,979 & 1,229 & \\
\hline
\end{tabular}

\section{VANCOUVER AND NORTHERN BRITISH COLUMBIA PORTS}

The Union Steamship Co. of British Columbia, Ltd.

Contract No. 18.

T. \& C. File No. 25801.

Tote 163.-Tancouver and Northern parts of British Columbia, steam service between-

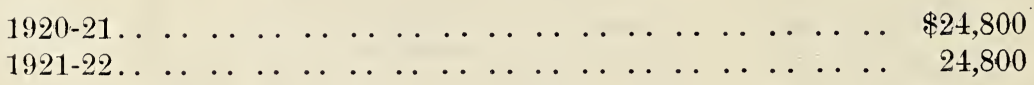

Contractors.-The Union Steamship Company of British Columbia, Ltd., Vancouver, B.C.

Date of Contract.-June 15, 1920.

Duration of Contract.-April 1, 1920, to March 31, 1921.

Service and Ports of Call.-Regular sailings throughout the year from Vancouver to Anyox (Granby Bay), on Observatory Inlet, making-

(a) Two calls each way each week at Campbell River, Port Hardy and Quathiasca Cove.

(b) One call each way each week at Alert Bay, Sointula, Bearer Cove, Shushartie Bay, Namu, Bella Bella, Bella Coola, Swanson Bay, Butedale, Prince Rupert, Port Simpson and Ocean Falls.

(c) One call each week at Wadhams, Hartley Bay, Port Essington, Arrandale, Kincolith, Mill Bay, Anyox, Claxton, and, weather permitting, at Suquash.

(d) One call one way every two weeks during summer and every four weeks during winter at Smith's Inlet. 
SESSIONAL PAPER No. $10 a$

(e) One call one way every four weeks at Kitimat.

( $f$ ) During the summer season one eall one way each week at Oceanic, China Hat, Lowe Inlet, Rivers Inlet, Schooner Passage, Kumeon and Alice Arm, and every two weeks in winter. Kimequit.

(g) During the summer season only one call one way every two weeks at

Under the present service to Bella Coola by the steamer making that place the terminal, one call per week suffices. If calls are made by the steamer operating through to Prince Rupert calls must be made each way each week. It is understood and agreed that the two calls each way each week at Port Hardy are conditional upon the Government float being installed at that port; otherwise one call each way each week will be sufficient.

Speed Required.-Not stated.

Subsidy. $\$ 24,800$ per annum, payable quarterly in July, October, January and April. (In addition to this, $\$ 9,200$ per annum is paid by the Post Office Department.)

Mails.-To be carried free. The contractors further agree to carry the mails to and from all ports at which they call, whether such call be stipulated in the agreement or not.

Government Wharves.-Steamers must call whenever possible.

DISTANCES.

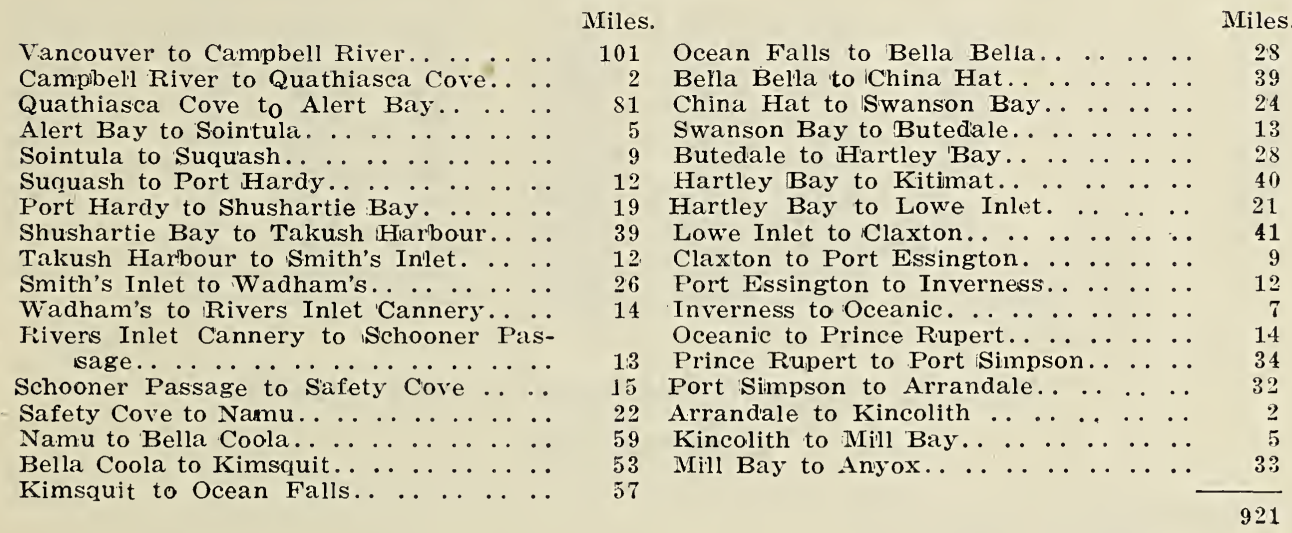


DESCRIPTION OF VESSELS EMPLOYED.

\begin{tabular}{|c|c|c|c|c|c|c|c|c|c|c|c|c|c|c|c|}
\hline \multirow[b]{2}{*}{ Name. } & \multicolumn{3}{|c|}{ Dimensions. } & \multicolumn{3}{|c|}{ Tonnage. } & \multicolumn{3}{|c|}{$\begin{array}{c}\text { Passenger } \\
\text { Accom- } \\
\text { modation. }\end{array}$} & \multirow{2}{*}{ 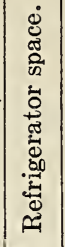 } & \multirow[b]{2}{*}{$\begin{array}{l}\text { Ai } \\
\dot{2} \\
\dot{z}\end{array}$} & \multirow[b]{2}{*}{ 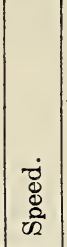 } & \multicolumn{3}{|c|}{ Built. } \\
\hline & 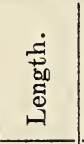 & 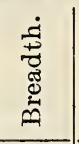 & 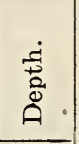 & $\begin{array}{l}\text { 莡 } \\
\end{array}$ & $\begin{array}{l}\dot{0} \\
\dot{0} \\
\dot{0} \\
\dot{0}\end{array}$ & 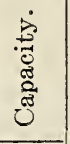 & 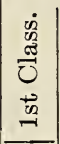 & 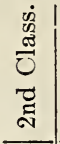 & 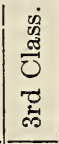 & & & & At & In & Of \\
\hline $\begin{array}{l}\text { Chelohsin... } \\
\text { Venture..... } \\
\text { Coquitlam.. } \\
\text { Casmosun... } \\
\text { Cowichan... } \\
\text { Chilkoot.... } \\
\text { Chilliwack.. }\end{array}$ & $\begin{array}{c}\text { Ft. } \\
175 \cdot 5 \\
180 \cdot 4 \\
120 \cdot 0 \\
192 \cdot 0 \\
156 \cdot 1 \\
170 \cdot 6 \\
172 \cdot 6\end{array}$ & \begin{tabular}{l|}
$\mathrm{Ft}$. \\
$35 \cdot 1$ \\
$32 \cdot 0$ \\
$22 \cdot 2$ \\
$35 \cdot 2$ \\
$32 \cdot 0$ \\
$27 \cdot 6$ \\
$30 \cdot 2$
\end{tabular} & \begin{tabular}{r|} 
Ft. \\
$15 \cdot 7$ \\
$17 \cdot 0$ \\
$9 \cdot 6$ \\
$17 \cdot 9$ \\
$13 \cdot 5$ \\
$10 \cdot 5$ \\
$12 \cdot 9$
\end{tabular} & $\begin{array}{l}597 \\
580 \\
165 \\
793 \\
520 \\
219 \\
410\end{array}$ & $\begin{array}{r}1,133 \\
1,011 \\
256 \\
1,369 \\
961 \\
557 \\
756\end{array}$ & $\begin{array}{l}479 \\
560 \\
357 \\
713 \\
565 \\
750 \\
800\end{array}$ & $\begin{array}{r}100 \\
100 \\
\text { Nil. } \\
100 \\
165 \\
\text { Nil. } \\
21\end{array}$ & $\begin{array}{r}91 \\
84 \\
\text { Nil. } \\
103 \\
\text { Niil. } \\
\text { Nil. }\end{array}$ & $\begin{array}{l}\text { Nil. } \\
\text { Nil. } \\
\text { Nil. } \\
\text { Nil. } \\
\text { Nil. } \\
\text { Nil. } \\
\text { Nil. }\end{array}$ & $\begin{array}{l}\text { C.ft } \\
\text { Nil. } \\
\text { Nil. } \\
\text { Nil. } \\
\text { Nil. } \\
\text { Nil. } \\
\text { Nil. } \\
\text { Nil. }\end{array}$ & $\begin{array}{r}131 \\
171 \\
28 \\
224 \\
151 \\
81 \\
95\end{array}$ & $\begin{array}{c}\text { Kts } \\
13 \frac{1}{2} \\
12 \\
7 \frac{1}{2} \\
11 \\
11 \\
9 \\
10\end{array}$ & $\begin{array}{l}\text { Dublin..... } \\
\text { Glasgow... } \\
\text { Vancouver. } \\
\text { Paisley .... } \\
\text { Ayr....... } \\
\text { Bowling... } \\
\text { N.Vancou- } \\
\text { ver...... }\end{array}$ & $\begin{array}{l}1911 \\
1910 \\
1892 \\
1905 \\
1908 \\
1903 \\
1920\end{array}$ & $\begin{array}{l}\text { Steel. } \\
\text { Steel. } \\
\text { Steel. } \\
\text { Steel. } \\
\text { Steel. } \\
\text { Steel. } \\
\text { Steel. }\end{array}$ \\
\hline
\end{tabular}

TRAFFIC RETURNS.

\begin{tabular}{|c|c|c|c|c|c|c|c|c|}
\hline \multirow{2}{*}{ Calendar Year. } & \multirow{2}{*}{$\begin{array}{c}\text { No. } \\
\text { of Round } \\
\text { Trips. }\end{array}$} & \multirow{2}{*}{$\begin{array}{c}\text { Passen- } \\
\text { gers } \\
\text { Carried. }\end{array}$} & \multicolumn{2}{|c|}{$\begin{array}{c}\text { Tons of Freight } \\
\text { Carried. }\end{array}$} & \multirow{2}{*}{$\begin{array}{l}\text { Live } \\
\text { Stock. }\end{array}$} & \multicolumn{2}{|c|}{ Mails. } & \multirow{2}{*}{$\begin{array}{l}\text { Subsidy } \\
\text { Paid. }\end{array}$} \\
\hline & & & Weight. & $\begin{array}{l}\text { Measure- } \\
\text { ment. }\end{array}$ & & $\begin{array}{l}\text { Lock } \\
\text { Bags. }\end{array}$ & $\begin{array}{l}\text { Tied } \\
\text { Sacks. }\end{array}$ & \\
\hline $\begin{array}{l}1912 \ldots \ldots \ldots \ldots \ldots \\
1913 \ldots \ldots \ldots \ldots \\
1914 \ldots \ldots \ldots \ldots \\
1915 \ldots \ldots \ldots \ldots \\
1916 \ldots \ldots \ldots \ldots \ldots \\
1917 \ldots \ldots \ldots \ldots \\
1918 \ldots \ldots \ldots \ldots \ldots \\
1919 \ldots \ldots \ldots \ldots \ldots \\
1920 \ldots \ldots \ldots \ldots \ldots\end{array}$ & $\begin{array}{l}217 \\
189 \\
209 \\
189 \\
241 \\
236 \\
268 \\
218 \\
234\end{array}$ & \begin{tabular}{|r|r|}
19,600 \\
19,328 \\
21,167 \\
15,126 \\
21,424 \\
23,453 \\
30,454 \\
27,309 \\
In 13,018 \\
Out 19,459
\end{tabular} & $\begin{array}{r}26,451 \\
99,106 \\
31,291 \\
30,627 \\
34,550 \\
31,011 \\
40,116 \\
31,474 \\
11,601 \\
8,615\end{array}$ & $\begin{array}{r}11,157 \\
45,433 \\
10,600 \\
8,541 \\
14,738 \\
13,511 \\
16,891 \\
12,169 \\
2,444 \\
12,512\end{array}$ & $\begin{array}{r}928 \\
791 \\
518 \\
602 \\
362 \\
309 \\
230 \\
165 \\
21 \\
221\end{array}$ & $\begin{array}{r}7,472 \\
8,042 \\
17,256 \\
13,831 \\
16,964 \\
19,526 \\
20,997 \\
22,103 \\
9,975 \\
16,897\end{array}$ & $\begin{array}{l}\text { Nil. } \\
2,828 \\
\text { Nil. } \\
\text { Nil. } \\
\text { Nil. } \\
\text { Nil. } \\
100 \\
\text { Nil. } \\
\text { Nil. } \\
\text { Nil. }\end{array}$ & $\begin{array}{r}\$ \text { cts. } \\
7,500 \\
15,100 \\
16,800 \\
16,800 \\
16,800 \\
16,800 \\
16,800 \\
16,800\end{array}$ \\
\hline $1920 \ldots \ldots \ldots \ldots$ & Total..... & 32,477 & 20,216 & 14,956 & 242 & 26,872 & Nil. & 22,800 \\
\hline
\end{tabular}

VANCOUVER AND PORTS ON HOWE SOUND

R. D. Thompson

Contract No. 78 .

T. \& C. File No. 26034.

Vote 164.-Vancouver and ports on Howe Sound, steam service between-

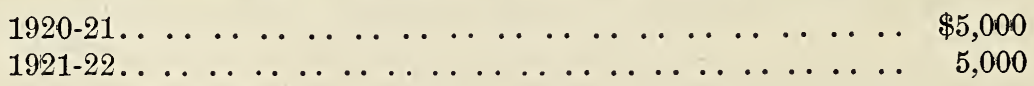

Contractor.-R. D. Thompson, of Vancouver, B.C.

Date of Contract.-August 24, 1920.

Duration of Contract.-April 1, 1920, to March 31, 1921.

Service and Ports of Call.-

(a) A regular daily service from April 1 to September 30 between Vancouver and Gibson's Landing, Hopkin's Landing, New Brighton, Port Mellon, Smith's Landing and Seaside Park. 
SESSIONAL PAPER No. 10a

(b) A regular service three times each week from October 1 to March 31, and more frequently should business warrant it, between Vancouver and Gibson's Landing, Hopkin's Landing, Smith's Landing and New Brighton.

(c) A regular service twice a week throughout the year between Vancouver and Hope Point (or Long Bay), West Bay, Grace Harbour, Elkin's Point, McNab Creek, Douglas, North Bay and Halkett Bay.

(d) A regular service twice a week from October 1 to March 31 between Vancouver, Port Mellon and Seaside Park, with more frequent trips, if business should warrant it.

\section{Speed Required.-Not stated.}

Subsidy. $-\$ 5,000$ per annum, payable quarterly.

Mails. - To be carried free. Mails to be receired and delivered at ship's side.

\section{DISTANCES.}

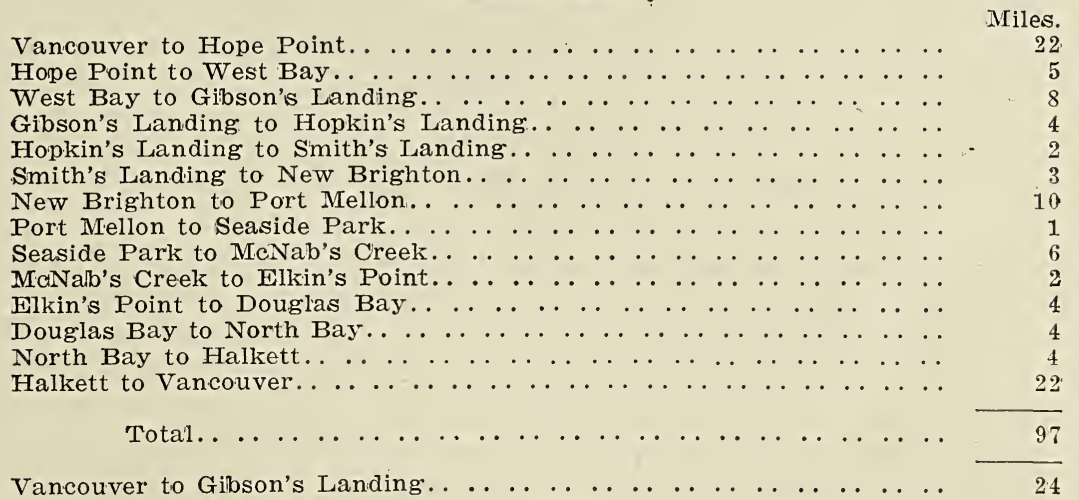

DESCRIPTION OF VESSEL EMPLOYED.

\begin{tabular}{|c|c|c|c|c|c|c|c|c|c|c|c|c|}
\hline \multirow[b]{2}{*}{ Name. } & \multicolumn{4}{|c|}{ Dimensions. } & \multicolumn{2}{|c|}{ Tonnage. } & \multirow{2}{*}{ 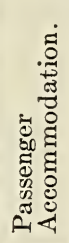 } & \multirow[b]{2}{*}{$\begin{array}{l}\dot{A} \\
\dot{H} \\
\dot{z}\end{array}$} & \multirow[b]{2}{*}{ 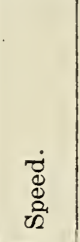 } & \multicolumn{3}{|c|}{ Built. } \\
\hline & 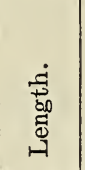 & 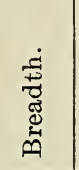 & $\begin{array}{l}\frac{d}{10} \\
\frac{0}{0} \\
0\end{array}$ & $\begin{array}{l}\dot{0} \\
\dot{0}\end{array}$ & $\begin{array}{l}\cdot \\
\dot{0} \\
0 \\
0 \\
\dot{0} \\
\dot{U}\end{array}$ & 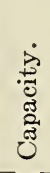 & & & & At & In & Of \\
\hline Britannia... & $\begin{array}{c}\text { Ft. } \\
104.8\end{array}$ & $\begin{array}{l}\text { Ft. } \\
22 \cdot 4\end{array}$ & $\begin{array}{l}\text { Ft. } \\
6 \cdot 09\end{array}$ & $221 \cdot 6$ & $325 \cdot 9$ & 60 & 200 & 33 & $\begin{array}{c}\text { Knot } \\
10\end{array}$ & Vancouver, B.C. & 1902 & Wood \\
\hline
\end{tabular}

TRAFFIC RETURNS

\begin{tabular}{|c|c|c|c|c|c|c|c|c|}
\hline \multirow[b]{2}{*}{ Period. } & \multirow[b]{2}{*}{$\begin{array}{l}\text { No. of } \\
\text { Round } \\
\text { Trips } \\
\text { run. }\end{array}$} & \multirow{2}{*}{\multicolumn{2}{|c|}{$\begin{array}{l}\text { Number of } \\
\text { Passengers } \\
\text { Carried. }\end{array}$}} & \multirow{2}{*}{$\begin{array}{l}\text { Tons of } \\
\text { Freight } \\
\text { Carried. }\end{array}$} & \multirow[b]{2}{*}{$\begin{array}{c}\text { Live } \\
\text { Stock. }\end{array}$} & \multicolumn{2}{|l|}{ Mails } & \multirow[b]{2}{*}{$\begin{array}{l}\text { Subsidy } \\
\text { Paid. }\end{array}$} \\
\hline & & & & & & $\begin{array}{l}\text { Lock } \\
\text { Bags. }\end{array}$ & $\begin{array}{c}\text { Tied } \\
\text { Sacks. }\end{array}$ & \\
\hline \multirow[t]{2}{*}{$\begin{array}{l}\text { Aug. } 1 \text { to Dec. } 31,1919 \text {. } \\
1920 \ldots \ldots \ldots \ldots \ldots \ldots \ldots \ldots \ldots\end{array}$} & \multirow[t]{2}{*}{$\begin{array}{l}114 \\
264\end{array}$} & $\begin{array}{l}\text { In } \\
\text { Out }\end{array}$ & $\begin{array}{l}5,378 \\
9,198 \\
9,405\end{array}$ & $\begin{array}{r}531 \\
110 \\
1,845\end{array}$ & $\begin{array}{l}48 \\
19 \\
43\end{array}$ & $\begin{array}{r}309 \\
911 \\
1,122\end{array}$ & $\begin{array}{r}464 \\
612 \\
1,642\end{array}$ & $\begin{array}{r}\$ \text { cts. } \\
1,74488 \\
5,00000\end{array}$ \\
\hline & & & 18,603 & 1,955 & 62 & 2,033 & 2,254 & \\
\hline
\end{tabular}





\section{LOCAL SERVICES.}

\section{BADDECK AND IONA}

The Baddeck Steamship Company, Lnhited

Contract No. 25.

T. \& C. File 25181.

Vote 165.-Baddeck and Iona, steam service between-

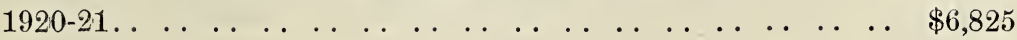

$$
\begin{aligned}
& 1921-22 \ldots \ldots \ldots \ldots \ldots \ldots \ldots \ldots \ldots \ldots \text {. . . . . . . . . . . . . . . . . . . }
\end{aligned}
$$

Contractors.-The Baddeck Steamship Company, Ltd., of Baddeck, N.S.

Contract Dated.-February 17, 1920.

Duration of Contract.-April 1, 1920, to Narch 31, 1921.

Service.-Two full round trips daily, during open navigation.

Ports of Call.-Baddeck, Iona and McKay's Point; calling at Kempt Head on the western end of Boularderie Island on trips from Baddeck to. Iona; such calls to be made only on those days on which the steamer Marion makes her westbound trip from Sydney to Whycocomagh; and calling at Grand Narrows whenever there is a reasonable amount of freight to take on or put off at that place.

Connections at Iona.-The steamer Blue Hill shall make connections at Iona with the afternoon eastbound train from Halifax to Sydney. In case the train is late in arriving at Iona, the steamer must wait for a reasonable time before proceeding to Baddeck.

Government Wharves.-Steamer must call whenever possible. April.

Subsidy. $-\$ 6,825$ per annum, payable quarterly, in July, October, January and

Mails.-To be carried free.

\section{DISTANCES.}

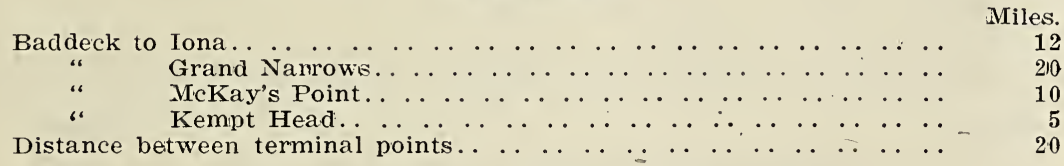

DESCRIPTION OF VESSEL EMPLOYED

\begin{tabular}{|c|c|c|c|c|c|c|c|c|c|c|c|c|}
\hline \multirow[b]{2}{*}{ Name. } & \multicolumn{3}{|c|}{ Dimensions. } & \multicolumn{3}{|c|}{ Tonnage. } & \multirow{2}{*}{ 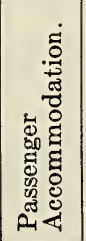 } & \multirow[b]{2}{*}{ 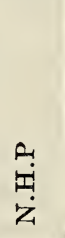 } & \multirow[b]{2}{*}{$\begin{array}{l}\dot{\Xi} \\
\dot{\Xi} \\
\text { भू. }\end{array}$} & \multicolumn{3}{|c|}{ Built. } \\
\hline & 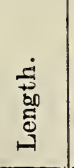 & 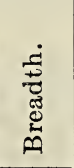 & 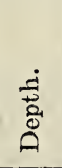 & $\stackrel{\dot{0}}{\ddot{z}}$ & 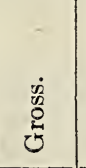 & 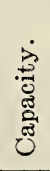 & & & & At & In & Of \\
\hline Blue Hill. & $\begin{array}{r}\text { Ft. } \\
135\end{array}$ & $\begin{array}{l}\text { Ft. } \\
18\end{array}$ & Ft. & 92 & $\begin{array}{l}\cdot \\
195\end{array}$ & & 500 & 38 & $\begin{array}{r}\text { Knots } \\
12\end{array}$ & $\begin{array}{l}\text { East Boston, } \\
\text { U.S.A. }\end{array}$ & 1887 & Wood \\
\hline
\end{tabular}


TRAFFIC RETURNS

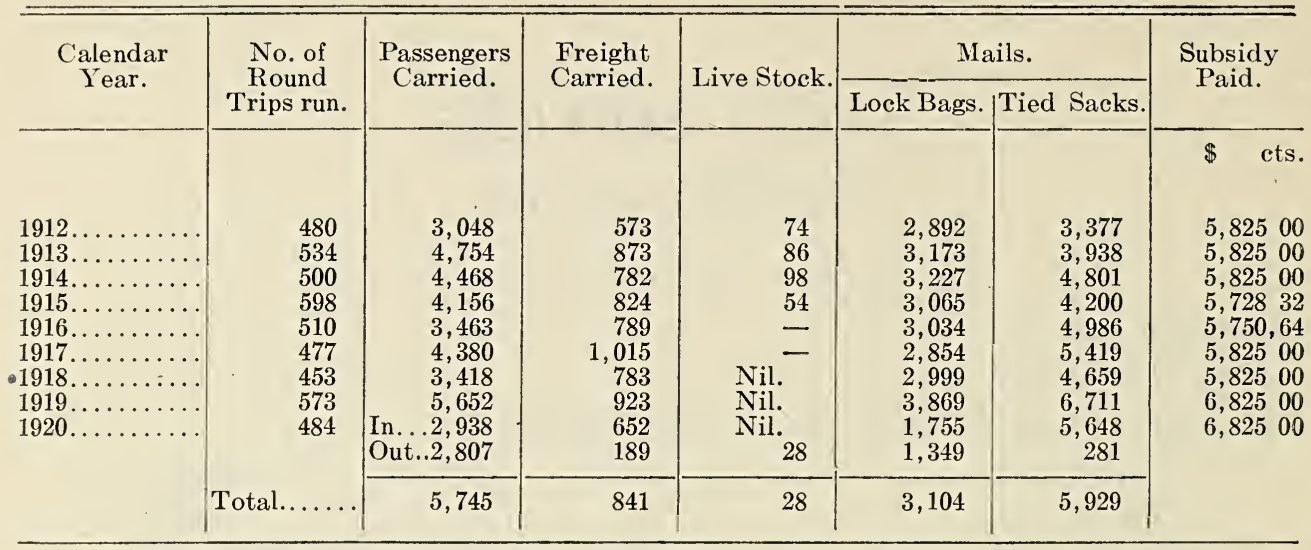

From 1907 to 1915, inclusive, and during the quarter ended March 31, 1916, the service was performed by the Victoria Steamship Company, of Baddeck, N.S., and their proportion of the subsidy shown above for 1916 was $\$ 1,475$.

\section{CHARLOTTETOWN, PICTOU AND NEW GLASGOW}

Vote 166.-Charlottetown, Pictou and New Glasgow, steam service between-

$$
\begin{aligned}
& 1920-21 \ldots \ldots \ldots \ldots \ldots \ldots
\end{aligned}
$$

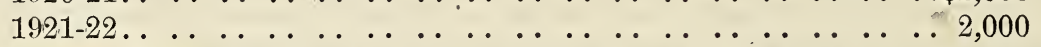

The Eastern Transport, Ltd., of New Glasgow, N.S., offered to perform a week?y service between the above-named ports, with the steamer Eastran, for an annual subsidy of $\$ 2,000$. The contract was never completed, however, as the company substituted for the Eastran an inferior vessel, the Trusty, with which an irregular service was performed. No subsidy has been paid yet, and no traffic returns furnished.

\section{CHARLOTTETOWN, VICTORIA AND HOLLIDAY'S WHARF}

$$
\text { Charlottetown Steamship Co. }
$$

Contract No. 74 .

T. \& C. File 25889.

Vote 16\%.-Charlottetown, Victoria and Holliday's Wharf, steam service between-

$$
\begin{aligned}
& 1920-21 \ldots \ldots \ldots \ldots \ldots \ldots 00
\end{aligned}
$$

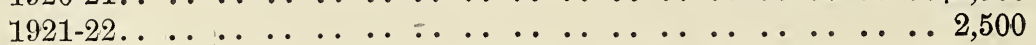

Contractors.-The Charlottetown Steamship Co., Ltd., of Charlottetown, P.E.I.

Contract dated.-July 6, 1920.

Duration of Contract.-From the opening to the close of navigation in 1920.

Service and Ports of Call-

Two round trips each week from the opening of navigation until October 1st, and thereafter one round trip each week until the close of navigation, from Charlottetown to Victoria; and two round trips each week throughout the season to Holliday's Wharf, East River and West River, calling at China Point, Orwell and Orwell Cove.

Subsidy. $-\$ 2,500$ per season, payable in two instalments

Mails.-To be carried free. 
SESSIONAL PAPER No. $10 a$

DISTANCES

Miles.

Charlottetown to Holliday's. . . . . . . . . . . . 17

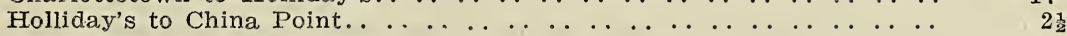

China Point to Orwell Cove. . . . . . . . . . . . . . . . . . . . . . .

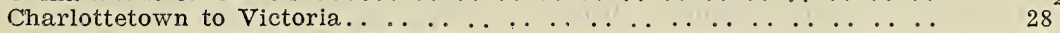

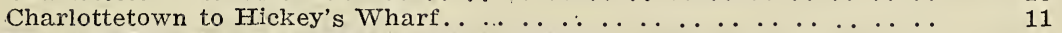

Hickey's Wharf to Haggarty's. . . . . . . . . . . . . . . . . . . . . . . . .

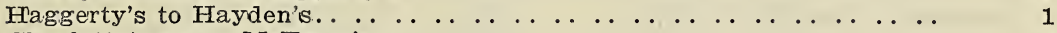

Charlottetown to McEwen's. . . . . . . . . . . . . . . . . . . . . . . . . .

McEwen's to West River Bridge.....................

DESCRIPTION OF VESSEL EMPLOYED

\begin{tabular}{|c|c|c|c|c|c|c|c|c|c|c|c|c|}
\hline \multirow[b]{2}{*}{ Name. } & \multicolumn{3}{|c|}{ Dimensions. } & \multicolumn{3}{|c|}{ Tonnage. } & \multirow{2}{*}{ 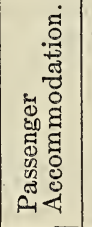 } & \multirow[b]{2}{*}{$\begin{array}{l}\text { Pi } \\
\text { 出 } \\
\dot{z}\end{array}$} & \multirow[b]{2}{*}{ 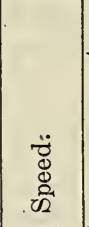 } & \multicolumn{3}{|c|}{ Built. } \\
\hline & 密 & 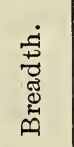 & 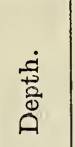 & 总 & 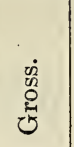 & 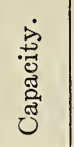 & & & & At & In & Of \\
\hline tos & $\begin{array}{c}\text { Ft. } \\
113\end{array}$ & $\begin{array}{r}\text { Ft. } \\
27\end{array}$ & \begin{tabular}{c|} 
Ft. \\
$6 \cdot 7$
\end{tabular} & 217 & 352 & 50 & 286 & 33 & \begin{tabular}{|r|} 
Knot \\
10
\end{tabular} & Shelburne, N.S. & 1908 & Wood \\
\hline
\end{tabular}

TRAFFIC RETURNS

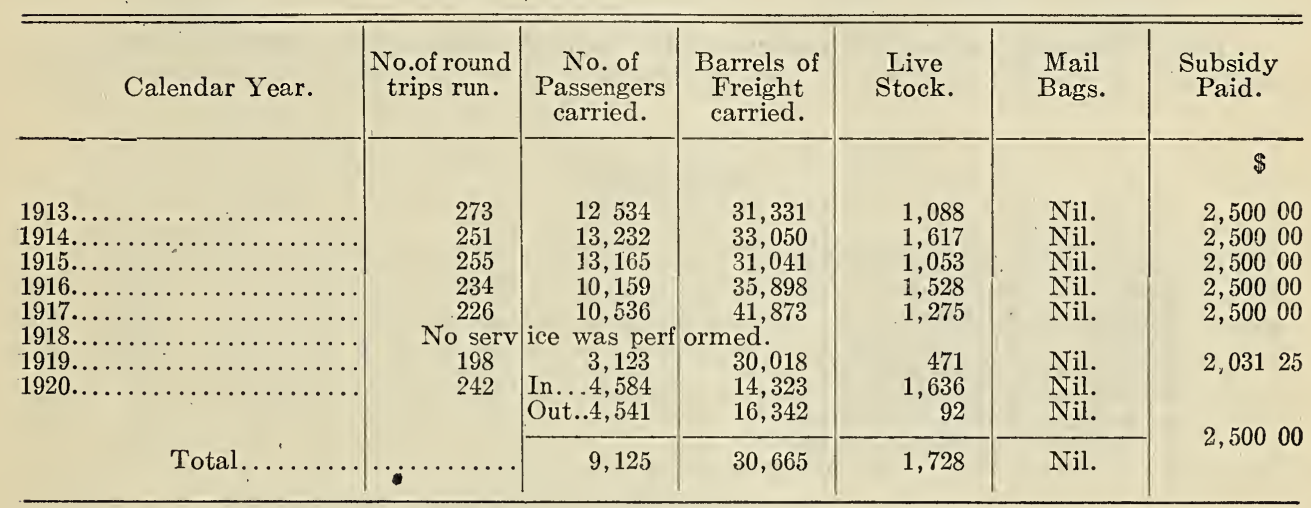

\section{GRAND MANAN AND THE MAINLAND}

Grand Manan Steamboat Company

Contract No. 14.

T. \& C. File No. 25759.

Vote 168.-Grand Manan and the Mainland, steam service between-

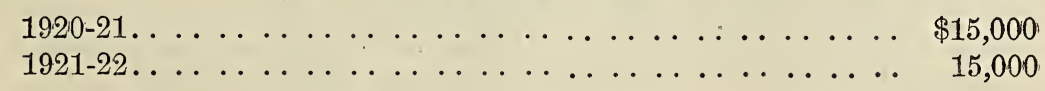

Contractors.-The Grand Manan Steamboat Company, of Grand Manan, N.B.

Date of Contract.-June 10, 1920.

I0a-3 
Duration of Contract.-April 1, 1920, to March 31, 1921.

Service and Ports of Call.-From June to September, inclusive:-

(a) One trip each week between Grand Manan and St. Andrew's, calling both ways at Campobello and Eastport, Maine.

(b) One trip each week between Grand Manan and St. John, via and calling both ways at Campobello and Eastport.

(c) One round trip each week between Grand Manan and St. John direct.

(d) One trip each week between Grand Manan and St. Stephen, calling both ways at Campobello, Eastport and St. Andrew's.

And during the remaining eight months of the year:-

(e) One trip each week between Grand Manan and St. Stephen, calling both ways at Campobello, Eastport and St. Andrew's.

( $f$ ) One trip each week between Grand Manan and St. John, calling both ways at Campobello and Eastport.

(g) One trip each week between Grand MTanan and St. Andrew's, calling both ways at Campobello and Eastport. April.

Subsidy.-\$15,000 per annum, payable quarterly in July, October, January and

Mails.-To be carried free.

Government Wharves.-Steamers must call at Government Wharves whenever possible.

DISTANCES.

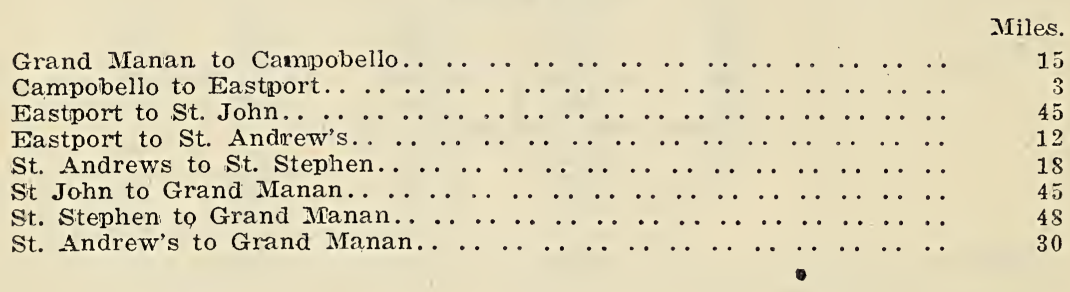

DESCRIPTION OFLSTEAMER EMPLOYED

\begin{tabular}{|c|c|c|c|c|c|c|c|c|c|c|c|c|}
\hline \multirow[b]{2}{*}{ Name., } & \multicolumn{3}{|c|}{ Dimensions. } & \multicolumn{3}{|c|}{ Tonnage. } & \multirow{2}{*}{ 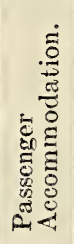 } & \multirow[b]{2}{*}{$\begin{array}{l}\dot{2} \\
\dot{z} \\
\dot{z}\end{array}$} & \multirow[b]{2}{*}{ 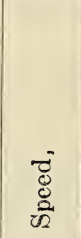 } & \multicolumn{3}{|c|}{ Built. } \\
\hline & 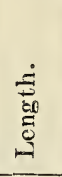 & 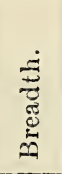 & 䒿 & 苛 & $\begin{array}{l}\dot{0} \\
\stackrel{0}{0} \\
\dot{0} \\
\dot{v}\end{array}$ & 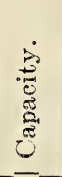 & & & & At & In & Of \\
\hline & Ft. & Ft. & Ft. & & & & & & Knots & & & \\
\hline Grand Manan. & 130 & 26 & 11 & 180 & 363 & 250 & 350 & 32 & 12 & Liverpool, N.S. & 1911 & Wood \\
\hline
\end{tabular}


TRAFFIC RETURNS.

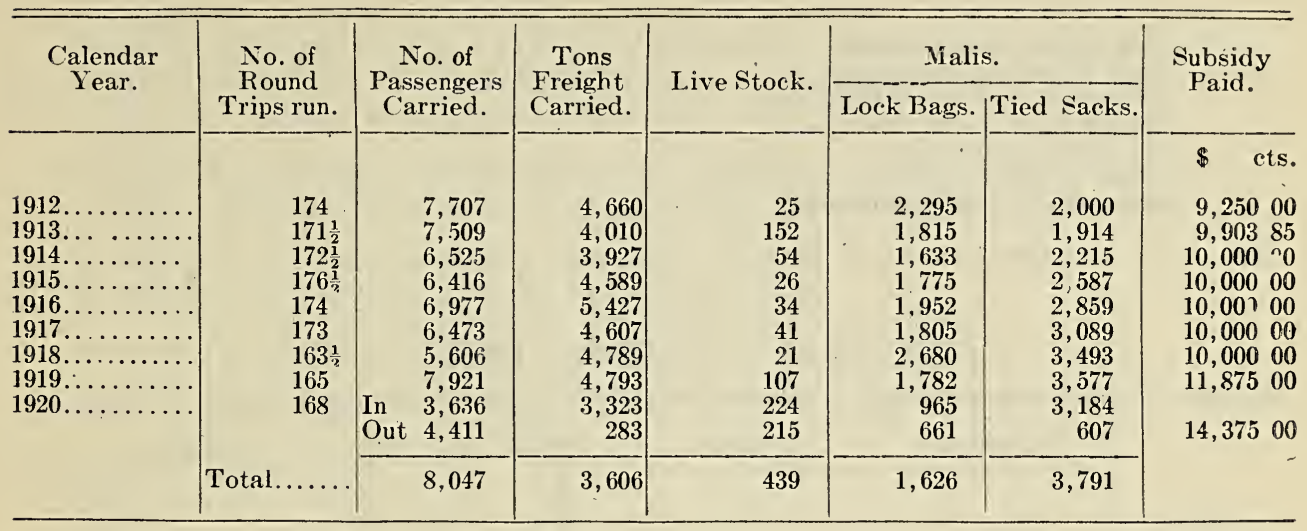

\section{HALIFAX, CANSO AND GUYSBOROUGH}

\section{Halifax and Canso Steanghip Co., Lts.}

Contract No. 30 .

T. \& C. File No. 25844.

Vote 169.-Halifax, Canso and Guysborough, steam service between$1920-21 \ldots \ldots \ldots \ldots \ldots \ldots$

$1921-22 \ldots \ldots \ldots \ldots \ldots \ldots$

Contractors.-The Halifax and Canso Steamship Co., Ltd., of Halifax, N.S.

Date of Contract.-June 23, 1920.

Duration of Contract.-April 1, 1920, to March 31, 1921.

Service.-Weekly, all the year round, between Halifax and Guysborough.

Ports of Call.-Calling on all voyages each way at Canso, Whitehead, Drumhead, Isaac's Harbour, Goldboro, Port Beckerton and, weather permitting, at Port Hilford; and calling on all outward voyages at Half Island Cove and Queensport; calling fortnightly during open navigation on outward royages at Country Harbour and Boylston; and during the months of January and February at Port Dufferin and Moser's River.

(a) From January 15 to March 31 a fortnightly call only need be made at Guysborough, Queensport and Half Island Cove.

(b) Calls at Port Hilford shall not be required when the depth of water south of the breakwater is less than 14 feet. The contractors shall, however, make every reasonable effort to call at this port.

Capacity of Steamer.-The steamer employed is guaranteed to have a cargo capacity equal to 2,500 barrels, with passenger accommodation for 40 passengers, electric lighted throughout and fitted with adequate refrigeration for the carriage of fresh fish.

Laying off Steamer.-The steamer may lay off for refitting two trips in each year, at such time or times as will least interfere with the requirements of the service.

Government wharves.-The steamer must call whenerer possible.

Subsidy. $\$ \$, 000$ per annum, payable quarterly in July, October, January and on the completion of the service.

Mails.-To be carried free.

$10 \mathrm{a}-3 \frac{1}{2}$ 
DISTANCES.

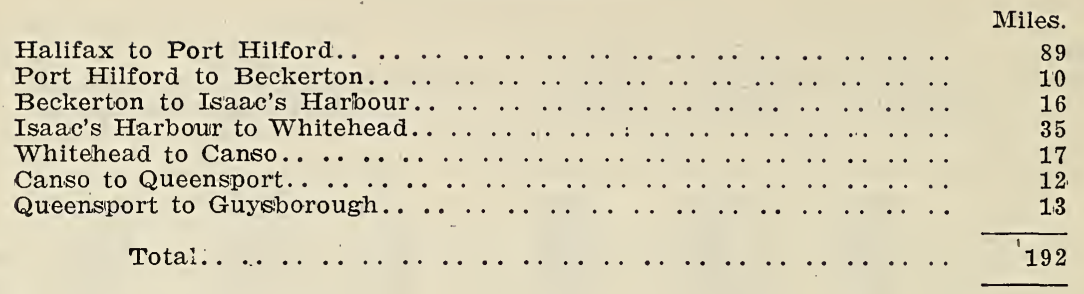

DESCRIPTION OF VESSEL EMPLOYED.

\begin{tabular}{|c|c|c|c|c|c|c|c|c|c|c|c|c|}
\hline \multirow[b]{2}{*}{ Name. } & \multicolumn{3}{|c|}{ Dimensions. } & \multicolumn{3}{|c|}{ Tonnage. } & \multirow{2}{*}{ 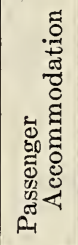 } & \multirow[b]{2}{*}{$\begin{array}{l}\dot{A} \\
\dot{u} \\
\dot{z}\end{array}$} & \multirow[b]{2}{*}{ 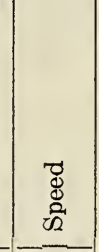 } & \multicolumn{3}{|l|}{ Built. } \\
\hline & 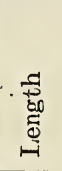 & 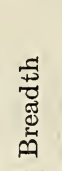 & 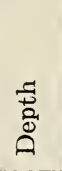 & $\begin{array}{l}\overrightarrow{0} \\
\ddot{z}\end{array}$ & $\begin{array}{l}0 \\
0 \\
0 \\
0 \\
0 \\
0\end{array}$ & 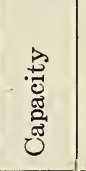 & & & & At & In & Of \\
\hline Scotia.. & $\begin{array}{l}\text { Ft. } \\
137\end{array}$ & $\begin{array}{r}\mathrm{Ft} . \\
27\end{array}$ & $\begin{array}{l}\text { Ft. } \\
9 \cdot 5\end{array}$ & 268 & 376 & brls. & 100 & 53 & $\begin{array}{c}\text { Knots } \\
10\end{array}$ & Mahone, N.S.. & 1907 & Wood \\
\hline
\end{tabular}

TRAFFIC RETURNS.

\begin{tabular}{|c|c|c|c|c|c|c|}
\hline Calendar Year. & $\begin{array}{l}\text { No.of round } \\
\text { trips run. }\end{array}$ & $\begin{array}{l}\text { No. of } \\
\text { Passengers } \\
\text { carried. }\end{array}$ & $\begin{array}{l}\text { Tons of } \\
\text { Freight } \\
\text { carried. }\end{array}$ & $\begin{array}{l}\text { Live } \\
\text { Stock. }\end{array}$ & $\begin{array}{l}\text { Mail } \\
\text { Bags. }\end{array}$ & $\begin{array}{l}\text { Subsidy } \\
\text { Paid. }\end{array}$ \\
\hline \multirow[t]{2}{*}{ 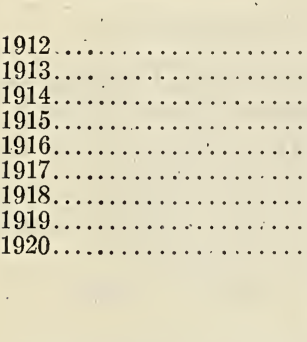 } & \multirow[t]{2}{*}{$\begin{array}{l}49 \\
51 \\
51 \\
51 \\
50 \\
51 \\
48 \\
50 \\
45\end{array}$} & $\begin{array}{r}1,344 \\
2,518 \\
2,377 \\
2,247 \\
2,475 \\
2,443 \\
2,168 \\
2,287 \\
\text { In } 902 \\
\text { Out } \quad 948\end{array}$ & $\begin{array}{r}21,369 \\
22,222 \\
22,731 \\
25,098 \\
19,862 \\
25,481 \\
23,869 \\
30,701 \\
3,928 \\
15,367\end{array}$ & $\begin{array}{l}\quad 25 \\
68 \\
\text { Nil. } \\
\text { Nil. } \\
\text { Nil. } 21 \\
\text { Nil. } \\
\text { Nil. } \\
\text { Nil. } \\
\text { Nil. }\end{array}$ & $\begin{array}{l}\text { Nil. } \\
\text { Nil. } \\
\text { Nil. } \\
\text { Nil. } \\
\text { Nil. } \\
\text { Nil. } \\
\text { Nil. } \\
\text { Nil. } \\
\text { Nil. }\end{array}$ & \multirow[t]{2}{*}{$\begin{array}{lr}\$ \quad \text { cts. } \\
4,00000 \\
4,75000 \\
5.00000 \\
5,00000 \\
5,00000 \\
5,00000 \\
5,00000 \\
5,00000 \\
6,096\end{array}$} \\
\hline & & 1,850 & 19,295 & Nir. & Nil. & \\
\hline
\end{tabular}

\section{HALIFAX AND LAHAVE RIVER PORTS}

The Western Steamship Co., Itd.

Contract No. 67.

T. \& C. File No. 25838.

Vote 170.-Halifax and LaHave River Ports, steam service between$1920-21 \ldots \ldots \ldots \ldots \ldots \ldots \ldots$. . . . . . . . . . . . . . . $\$ 4,000$

$1921-22 \ldots \ldots \ldots \ldots \ldots \ldots \ldots$

Contractors.-The Western Steamship Co., Ltd., Halifax, N.S.

Date of Contract.-June 23, 1920. 
SESSIONAL PAPER No. 10a

Duration of Contract.-Opening of navigation, 1920, to March 31, 1921.

Service and Ports of Call.-Leaving Halifax once each week, calling at LaHave, Riverport, East LaHave, Pleasantville, Conquerall Bank and Dayspring, and returning to Halifax, calling at the aforesaid ports.

During the winter months, when the LaHave river is frozen over, calls at Pleasantville, East LaHave, Conquerall Bank and Dayspring may be omitted.

During the months of January and February calls at any of the said ports may be omitted if ice conditions prevent their being made.

Government Wharves.-Steamers must call whenever possible.

Subsidy. $\$ 4,000$ per annum, payable in quarterly instalments on the last days of June, September, December and March.

Mails.-To be carried free.

Withdrawal of steamer for repairs.-The steamer may be withdrawn from the service for a total period of fourteen days, if required, for necessary repairs.

DISTANCES.

Miles.

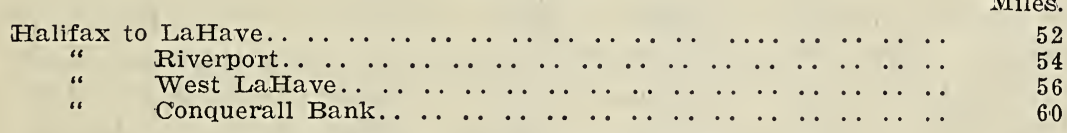

DESCRIPTION OF VESSEL EMPLOYED

\begin{tabular}{|c|c|c|c|c|c|c|c|c|c|c|c|c|c|}
\hline \multirow[b]{2}{*}{ Name. } & \multicolumn{3}{|c|}{ Dimensions. } & \multicolumn{3}{|c|}{ Tonnage. } & \multirow{2}{*}{ 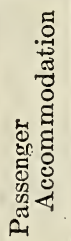 } & \multirow[b]{2}{*}{$\begin{array}{l}\dot{A} \\
\dot{z} \\
\dot{z}\end{array}$} & \multirow[b]{2}{*}{ 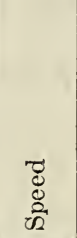 } & \multicolumn{4}{|c|}{ Built. } \\
\hline & 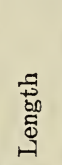 & 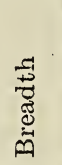 & 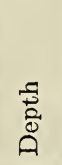 & 苟 & 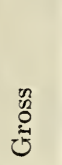 & 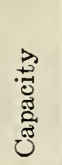 & & & & At & & In & Of \\
\hline Enterprise & $\begin{array}{l}\text { Ft. } \\
108\end{array}$ & $\underset{25}{\mathrm{Ft}}$ & $\begin{array}{l}\text { Ft. } \\
8.6\end{array}$ & 98 & 211 & 100 & 100 & 42 & $\begin{array}{c}\text { Knots } \\
11\end{array}$ & Shelburne, & N.S. & 1907 & Wood. \\
\hline
\end{tabular}

TRAFFIC RETURNS

\begin{tabular}{|c|c|c|c|c|c|c|}
\hline Calendar Year. & $\begin{array}{c}\text { No. of round } \\
\text { trips run. }\end{array}$ & $\begin{array}{l}\text { No. of } \\
\text { Passengers } \\
\text { carried. }\end{array}$ & $\begin{array}{l}\text { Tons of } \\
\text { Freight } \\
\text { carried. }\end{array}$ & $\begin{array}{l}\text { Live } \\
\text { Stock. }\end{array}$ & $\begin{array}{l}\text { Bags } \\
\text { Mail }\end{array}$ & $\begin{array}{c}\text { Subsidy } \\
\text { Paid. }\end{array}$ \\
\hline \multirow[t]{2}{*}{$\begin{array}{l}1912 . . \\
1913 . \\
1914 . \\
1915 . \\
1916 . \\
1917 . \\
1918 . \\
1919 . \\
1920 . .\end{array}$} & $\begin{array}{l}43 \\
88 \\
68 \frac{1}{2} \\
76 \frac{1}{2} \\
83 \frac{1}{2} \\
72 \\
23 \\
44 \\
46\end{array}$ & $\begin{array}{lc} & 410 \\
& 526 \\
& \text { Nil } \\
& 134 \\
& 682 \\
& 885 \\
& 368 \\
& 343 \\
\text { In } & 119 \\
\text { Out } & 127\end{array}$ & $\begin{array}{r}360 \\
5,900 \\
4,294 \\
4,820 \\
5,287 \\
7,310 \\
2,300 \\
3,807 \\
592 \\
2,690\end{array}$ & $\begin{array}{l}12 \\
\text { Nil. } \\
\text { Nil. } \\
\text { Nil. } \\
\text { N } \\
\text { Nil. } \\
\text { Nil. } \\
\text { Nil. } \\
\text { Nil. } \\
\text { Nil. }\end{array}$ & $\begin{array}{l}\text { Nil. } \\
\text { Nil. } \\
\text { Nil. } \\
\text { Nil. } \\
\text { Nil. } \\
\text { Nil. } \\
\text { Nil. } \\
\text { Nil. } \\
\text { Nil. } \\
\text { Nil. }\end{array}$ & $\begin{array}{lr}\$ & \text { cts. } \\
3,147 & 73 \\
3,153 & 85 \\
2,815 & 00 \\
3,000 & 77 \\
3,639 & 60 \\
4,513 & 57 \\
1,095 & 26 \\
2,250 & 00 \\
3,750 & 00\end{array}$ \\
\hline & Total....... & 246 & 3,282 & Nil. & Nil. & \\
\hline
\end{tabular}




\section{HALIFAX AND NEWFOUNDLAND via CAPE BRETON PORTS}

J. A. FARQuHAR \& CO., LTD.

Contract No. 11.

T. \& C. File No. 25956.

Vote 171.-Halifax and Newfoundland via Cape Breton ports, steam service between-

$$
\begin{aligned}
& 1920-21 \ldots \ldots \ldots \ldots \ldots \ldots \ldots
\end{aligned}
$$

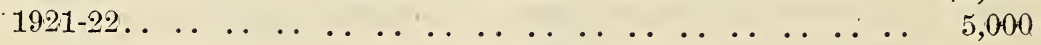

Contractors.-J. A. Farquhar \& Co., Ltd., of Halifax, N.S.

Date of Contract:-July 26, 1920.

Duration of Contract.-For the season of navigation, 1920.

Service-Fornightly, until 14 complete round trips have been performed; or until the close of navigation, should it close before the said 14 trips can be performed.

Ports of Call.-Halifax to Sydney, via the south shore of Cape Breton, thence to North Sydney, Marble Mountain, Baddeck, Ingonish, Neil's Harbour, White Point and St. Paul's Island; thence to Channel, Codroy, Sandy Point, Bay of Islands and Bonne Baie, Nfld.; thence returning to Halifax, calling at Bay of Islands, Sandy Point, Codroy, Channel, St. Paul's Island, White Point, Neill's Harbour, Ingonish, North Sydney and Sydney.

Sudsidy. $-\$ 5,000$ for the season, payable at the rate of $\$ 357.14$ per round trip.

Mails.-To be carried free.

\section{DISTANCES}

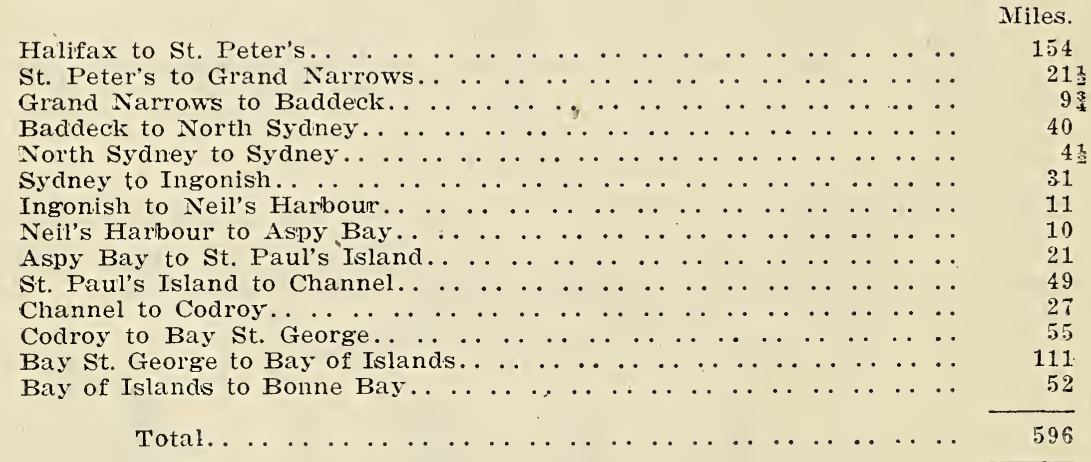


DESCRIPTION OF VESSEL EMPLOYED.

\begin{tabular}{|c|c|c|c|c|c|c|c|c|c|c|c|c|}
\hline \multirow{2}{*}{ Name. } & \multicolumn{3}{|c|}{ Dimensions. } & \multicolumn{3}{|c|}{ Tonnage. } & \multirow{2}{*}{ 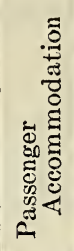 } & \multirow[b]{2}{*}{$\begin{array}{l}\dot{2} \\
\dot{z} \\
\dot{z}\end{array}$} & \multirow[b]{2}{*}{ 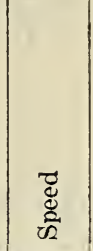 } & \multicolumn{3}{|c|}{ Built. } \\
\hline & 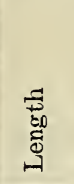 & 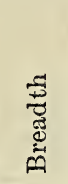 & $\frac{\mathfrak{f}}{\stackrel{0}{0}}$ & $\begin{array}{l}\overrightarrow{0} \\
\ddot{z}\end{array}$ & $\begin{array}{l}\text { n } \\
\text { d } \\
0 \\
0\end{array}$ & 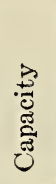 & & & & At & In & Of \\
\hline Stelia Maris : & $\begin{array}{l}\text { Ft. } \\
124\end{array}$ & $\begin{array}{l}\text { Ft. } \\
23 \cdot 6\end{array}$ & $\begin{array}{l}\text { Ft. } \\
12 \cdot 2\end{array}$ & 54 & 229 & 350 & Nil. & 70 & $\begin{array}{r}\text { Knots } \\
8 \frac{1}{2}\end{array}$ & London. & 1882 & $\begin{array}{l}\text { Wood } \\
\text { and } \\
\text { iron }\end{array}$ \\
\hline
\end{tabular}

TRAFFIC RETURNS.

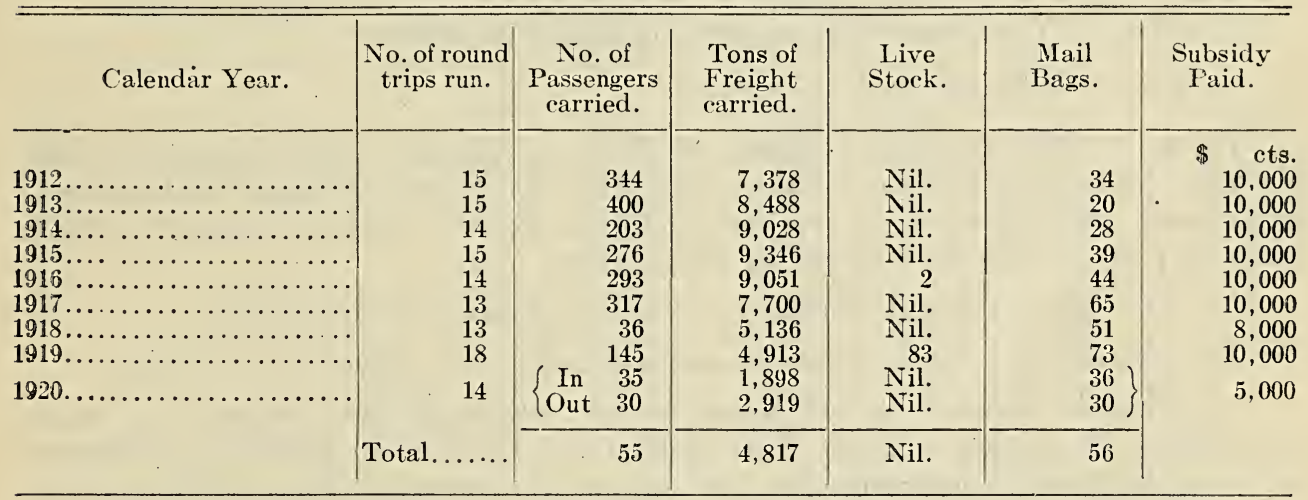

From 1907 to 1910 inclusive, the service was performed by Messrs. Pickford \& Black, of Halifax, N.S.

ORIGIN, QUANTITY AND VALUE OF FREIGHT EXPORTED FROM CANADA TO NEWFOUNDLAND

\begin{tabular}{|c|c|c|c|c|c|c|c|c|c|}
\hline \multirow{2}{*}{ Calendar Year. } & \multicolumn{3}{|c|}{ Canadian Origin. } & \multicolumn{3}{|c|}{ United States Origin. } & \multicolumn{3}{|c|}{ Total. } \\
\hline & Weight. & $\begin{array}{l}\text { Measure- } \\
\text { ment. }\end{array}$ & Value. & Weight. & $\begin{array}{l}\text { Measure- } \\
\text { ment. }\end{array}$ & Value. & Weight. & $\begin{array}{l}\text { Measure- } \\
\text { ment. }\end{array}$ & Value \\
\hline $\begin{array}{l}1912 \ldots \ldots \ldots \ldots \ldots \\
1913 \ldots \ldots \ldots \ldots \ldots \ldots \\
1914 \ldots \ldots \ldots \ldots \ldots \\
1915 \ldots \ldots \ldots \ldots \ldots \\
1916 \ldots \ldots \ldots \ldots \ldots \\
1917 \ldots \ldots \ldots \ldots \ldots \\
1918 \ldots \ldots \ldots \ldots \ldots \ldots \\
1919 \ldots \ldots \ldots \ldots \ldots \ldots \\
1920 \ldots \ldots \ldots \ldots \ldots\end{array}$ & \begin{tabular}{r|} 
Tons. \\
2,366 \\
3,091 \\
4,421 \\
4,308 \\
4,012 \\
3,058 \\
2,280 \\
2,631 \\
2,565
\end{tabular} & $\begin{array}{r}\text { Tons. } \\
36 \\
636 \\
100 \\
167 \\
73 \\
53 \\
34 \\
83 \\
\text { Nil. }\end{array}$ & $\begin{array}{c}\$ \\
119,300 \\
214,938 \\
217,962 \\
257,505 \\
276,533 \\
293,323 \\
246,535 \\
410,650 \\
374,210\end{array}$ & \begin{tabular}{|r|} 
Tons. \\
1,139 \\
552 \\
178 \\
293 \\
690 \\
454 \\
300 \\
621 \\
354
\end{tabular} & $\begin{array}{r}\text { Tons. } \\
121 \\
554 \\
129 \\
278 \\
12 \\
3 \\
3 \\
\text { Nil. } \\
\text { Nil. }\end{array}$ & $\begin{array}{r}\$ \\
90,743 \\
37,799 \\
25,925 \\
46,341 \\
110,740 \\
75,290 \\
43,121 \\
73,232 \\
69,502\end{array}$ & \begin{tabular}{|r|} 
Tons. \\
3,505 \\
3,643 \\
4,599 \\
4,601 \\
4,702 \\
3,512 \\
2,580 \\
3,252 \\
2,919
\end{tabular} & $\begin{array}{r}\text { Tons. } \\
157 \\
1,188 \\
229 \\
445 \\
85 \\
56 \\
37 \\
83 \\
\text { Nil. }\end{array}$ & $\begin{array}{r}\$ \text { cts. } \\
210,043 \\
252,737 \\
243,887 \\
303,846 \\
387,273 \\
368,613 \\
291,656 \\
483,882 \\
443,712\end{array}$ \\
\hline
\end{tabular}

\section{PRINCIPAL ARTICLES EXPORTED}

Of Canadian Origin.-Flour, paint, molasses, gasolene, beans, stoves, furniture, butter, tea, hay, rope, tin ingots, apples, oats, kerosene oil, beef, pork, sugar, potatoes, oil, clothing, boots and shoes, nets, hardware, wire fencing, roofing, axes, and engines.

Of United States and Foreign Origin.-Molasses, engines and parts thereof, beef, rope, machinery, pork, tobacco, kerosene oil, oranges, shoes, feed, whisky, sugar, tea, salt, gasolene, engines, bananas, oranges, and raisins. 


\section{HALIFAX, SPRY BAY AND CAPE BRETON PORTS}

Halifax and Sheet Harbour Steamship Company, Ltd.

Contract No. 55 .

T. \& C. File No. 25479.

Vote 172.-Halifax and Spry Bay and ports in Cape Breton, steam service between-

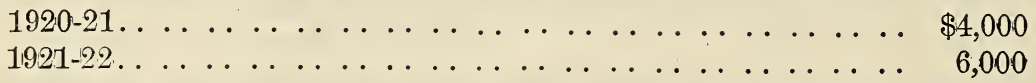

Contractors.-Halifax and Sheet Harbour Steamship Company, Ltd., 234-246 Hollis street, Halifax, N.S.

Date of Contract.-April 13, 1920.

Duration of Contract.-April 1, 1920, to March 31, 1921.

Service.-Weekly, all the year round.

Ports of Call.-Halifax, Jeddore, Owl's Head, Tangier, Pope's Harbour, Ship Harbour, Sheet Harbour, Sober Island, Spry Bay, St. Peter's, Arichat, West Arichat, Poulamond and L'Ardoise; as well as such other ports or places between the above terminal ports as may be required by the minister.

(a) The call at Pope's Harbour is at the discretion of the contractors.

(b) The call at L'Ardoise may be omitted in January, February and March.

(c) Between January 20 and March 20 the contractors have the option of omit. ting calls at St. Peter's, Arichat, West Arichat and Poulamond, provided satisfactory proof can be shown the minister that the steamer was prevented from calling at these ports on account of ice conditions.

(d) A fortnightly service to Marble Mountain shall be performed during the season of open navigation.

(e) Through bills of lading shall be issued by the Margaret between Halifax and all ports in the Bras d'Or lakes and on the eastern coast of Cape Breton called at by the Arcadia during the present season.

Subsidy. $-\$ 4,000$ per annum, payable in four equal instalments in July, October, January and April.

Government Wharves.-Steamer must call whenever possible.

Time for repairs.-Three weeks are allowed during the year for Government inspection and annual overhauling.

Mails.-To be carried free.

DISTANCES

Miles.

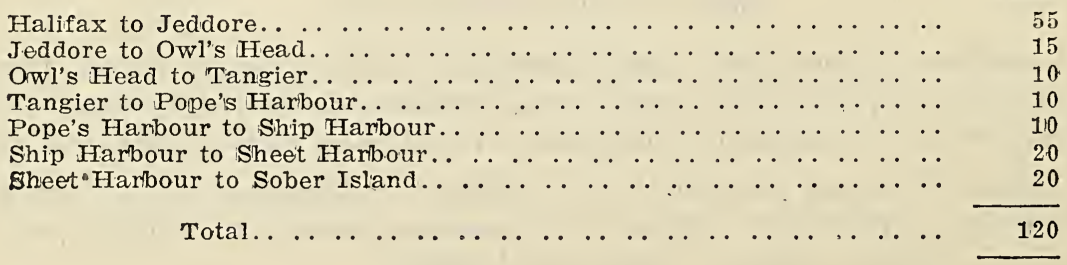




\title{
DESCRIPTION OF VESSEL EMPLOYED
}

\begin{tabular}{|c|c|c|c|c|c|c|c|c|c|c|c|c|c|}
\hline \multirow[b]{2}{*}{ Name. } & \multicolumn{3}{|c|}{ Dimensions. } & \multicolumn{3}{|c|}{ Tonnage. } & \multirow{2}{*}{ 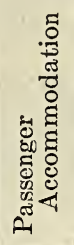 } & \multirow[b]{2}{*}{$\begin{array}{l}\dot{A} \\
\dot{1} \\
\dot{z}\end{array}$} & \multirow[b]{2}{*}{$\begin{array}{l}\mathbb{8} \\
\& \\
\frac{\mathbb{2}}{W 2}\end{array}$} & \multicolumn{4}{|c|}{ Built. } \\
\hline & 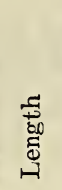 & 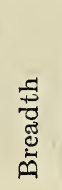 & $\begin{array}{l}\text { 蒂 } \\
\text { مी }\end{array}$ & $\begin{array}{l}\overrightarrow{0} \\
z \\
\ddot{z}\end{array}$ & 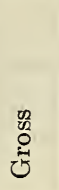 & 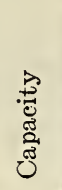 & & & & 1 & At & In & Of \\
\hline Margaret. & $\begin{array}{r}\mathrm{Ft} . \\
92\end{array}$ & $\begin{array}{l}\mathrm{Ft} . \\
19\end{array}$ & Ft. & 100 & 195 & 175 & 90 & 27 & $\begin{array}{c}\text { Knots } \\
10 \frac{1}{2}\end{array}$ & Sheet & Harbour. & 1907 & Wood \\
\hline
\end{tabular}

TRAFFIC RETURNS

\begin{tabular}{|c|c|c|c|c|c|c|}
\hline Calendar Year. & $\begin{array}{l}\text { No.of round } \\
\text { trips run. }\end{array}$ & $\begin{array}{c}\text { No. of } \\
\text { Passengers } \\
\text { carried. }\end{array}$ & $\begin{array}{l}\text { Tons of } \\
\text { Freight } \\
\text { carried. }\end{array}$ & $\begin{array}{l}\text { Live } \\
\text { Stock. }\end{array}$ & Mail. & $\begin{array}{l}\text { Subsidy } \\
\text { Paid. }\end{array}$ \\
\hline 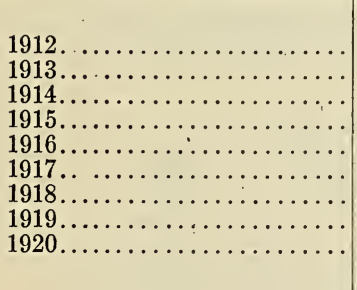 & $\begin{array}{r}52 \\
50 \\
50 \\
50 \\
45 \\
45 \\
41 \\
46 \\
46\end{array}$ & $\begin{array}{r}3,138 \\
3,244 \\
2,572 \\
2,630 \\
2,207 \\
1,522 \\
1,101 \\
1,342 \\
\text { In } \quad 920 \\
\text { Out } 1,059\end{array}$ & $\begin{array}{l}7,380 \\
7,430 \\
7,700 \\
7,535 \\
9,180 \\
7,155 \\
5,740 \\
6,225 \\
1,725 \\
3,875\end{array}$ & $\begin{array}{l}\text { Nil. } \\
\text { Nil. } \\
\text { Nil. } \\
\text { Nil. } \\
\text { Nil. } \\
\text { Nil. } \\
\text { Nil. } \\
\text { Nil. } \\
\text { Nil. }\end{array}$ & $\begin{array}{l}\text { Nil. } \\
\text { Nil. } \\
\text { Nil. } \\
\text { Nil. } \\
\text { Nil. } \\
\text { Nil. } \\
\text { Nil. } \\
\text { Nil. } \\
\text { Nil. } \\
\text { Nil. }\end{array}$ & $\begin{array}{lr}\$ & \text { cts. } \\
2,500 & 00 \\
2,153 & 84 \\
3,000 & 00 \\
4,000 & 00 \\
3,603 & 08 \\
3,750 & 25 \\
3,767 & 92 \\
4,000 & 00 \\
4,000 & 00\end{array}$ \\
\hline & Total .... & 1,979 & 5,600 & Nil. & Nil. & \\
\hline
\end{tabular}

\section{HALIFAX, SOUTH CAPE BRETON AND BRAS D'OR LAKE PORTS}

\author{
HENDRY, LTD.
}

Contract No. 66.

T. \& C. File No. 25480 .

Vote 173.-Halifax, South Cape Breton and Bras d'Or Lalie Ports, steam service between-

$$
\begin{array}{rllllllllllllllllll}
1020-21 \ldots & \ldots & \ldots & \ldots & \ldots & \ldots & \ldots & \ldots & \ldots & \ldots & \ldots & \ldots & \ldots & \ldots & \ldots & \ldots & \ldots & \$ 6,000 \\
1921-22 \ldots & \ldots & \ldots & \ldots & \ldots & \ldots & \ldots & \ldots & \ldots & \ldots & \ldots & \ldots & \ldots & \ldots & \ldots & \ldots & \ldots & \ldots & 6,000
\end{array}
$$

Contractors.-Messrs. Hendry, Ltd., Halifax, N.S.

Date of Contract.-April 13, 1920.

Duration of Contract.-From the opening to the close of navigation in 1920.

Service and Ports of.Call.-Passengers and freight shall be conveyed between Halifax and St. Peter's by the steamers Margaret and Strathlorne, and shall be transshipped at St. Peter's to and from the Arcadia for the purpose of proceeding to or from the under-mentioned points in the Bras d'Or lakes, and on the east coast of Cape Breton. The Arcadia's services shall be as follows: (a) Four round trips each month from St. Peter's, N.S., to Sydney, through the Bras d'Or Lakes, calling at all ports on the east and west sides of the lakes at which freight is offered, or is to be delivered, including Grand Narrows, Iona, Baddeck, Whycocomagh, Little Narrows, Nyanza, Boularderie Centre, Boularderie, Marble Mountain, West Bay, Washabuck Centre, Johnston's Harbour, Irish Cove, Big Pond, East Bay, Castle Bay and Grand Narrows, extending each trip from Sydney to North Sydney, Glace Bay, Port Morien, Mainadieu, Louisburg and Gabarous. 
Through tickets for passengers and through bills of lading for freight shall be issued between Halifax via the Margaret and Strathlorne, and the above-mentioned ports in the Bras d'Or Lakes and on the East Coast of Cape Breton.

If, during the early spring and late fall, the steamers Strathlorne and Margaret have their full complement of freight and are unable to carry the additional freight required to and from the Arcadia, the contractors agree to supply an additionai steamer between Halifax and St. Peter's, for the purpose of carrying the surplus of the Arcadia's freight.

The calls at Mainadieu are to be conditional upon the weather permitting. Calls at Glace Bay shall not be made unless, in the opinion of the Minister, it is safe for the steamer Arcadia to call at this port.

Government Wharves.-Steamer must call whenever possible.

Subsidy. $-\$ 6,000$ per annum, payable in four instalments of $\$ 1,500$ each.

Mails.- $\mathrm{T}_{0}$ be carried free.

DISTANCES.

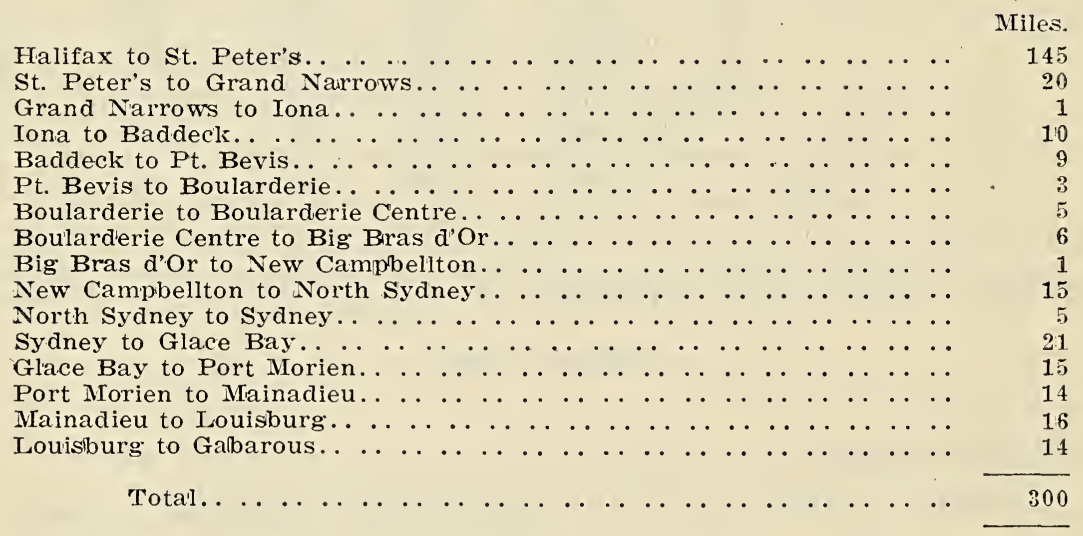

DESCRIPTION OF VESSEL EMPLOYED

\begin{tabular}{|c|c|c|c|c|c|c|c|c|c|c|c|c|}
\hline \multirow[b]{2}{*}{ Name. } & \multicolumn{3}{|c|}{ Dimensions. } & \multicolumn{3}{|c|}{ Tonnage. } & \multirow{2}{*}{ 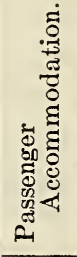 } & \multirow[b]{2}{*}{$\begin{array}{l}\dot{4} \\
\dot{1} \\
\dot{z}\end{array}$} & \multirow[b]{2}{*}{ 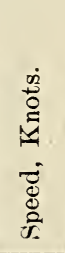 } & \multicolumn{3}{|l|}{ Built. } \\
\hline & 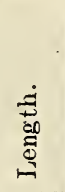 & 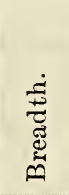 & 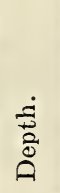 & $\frac{+0}{\ddot{c}}$ & $\begin{array}{l}\dot{0} \\
\dot{0} \\
\dot{0} \\
\dot{U}\end{array}$ & 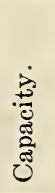 & & & & At & In & Of \\
\hline Arcadia....... & $\begin{array}{r}\text { Ft. } \\
73\end{array}$ & $\begin{array}{l}\text { Ft. } \\
16 \cdot 7\end{array}$ & $\begin{array}{l}\text { Ft. } \\
6 \cdot 4\end{array}$ & 42 & 62 & 70 & 40 & 30 & 8 & Yarmouth, N.S. & 1884 & Wood. \\
\hline
\end{tabular}


TRAFFIC RETURNS.

\begin{tabular}{|c|c|c|c|c|c|c|}
\hline Calendar Year. & $\begin{array}{c}\text { No. of round } \\
\text { trips run. }\end{array}$ & $\begin{array}{l}\text { No. of } \\
\text { Passengers } \\
\text { carried. }\end{array}$ & $\begin{array}{l}\text { Tons of } \\
\text { Freight } \\
\text { carried. }\end{array}$ & $\begin{array}{l}\text { Live } \\
\text { Stock. }\end{array}$ & $\begin{array}{l}\text { Bags } \\
\text { Mail. }\end{array}$ & $\begin{array}{l}\text { Subsidy } \\
\text { Paid. }\end{array}$ \\
\hline \multirow[t]{2}{*}{ 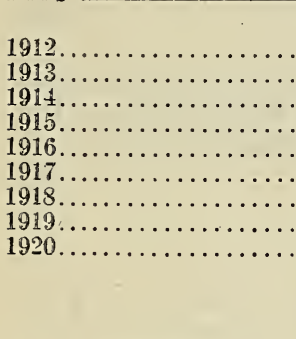 } & $\begin{array}{l}21 \\
21 \\
19 \\
14 \\
15 \\
15 \\
14 \\
26 \\
32\end{array}$ & 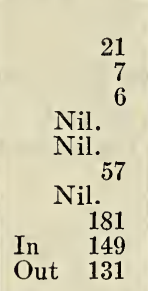 & $\begin{array}{l}4,985 \\
4,930 \\
4,603 \\
4,290 \\
3,770 \\
4,270 \\
2,342 \\
3,775 \\
2,910 \\
2,910\end{array}$ & $\begin{array}{l}\text { Nil. } \\
\text { Nil. } \\
\text { Nil. } \\
\text { Nil. } \\
\text { Nil. } \\
\text { Nil. } \\
\text { Nil. } \\
\text { Nil. } \\
\text { Nil. } \\
\text { Nil. }\end{array}$ & $\begin{array}{l}\text { Nil. } \\
\text { Nil. } \\
\text { Nil. } \\
\text { Nil. } \\
\text { Nil. } \\
\text { Niı. } \\
\text { Nil. } \\
\text { Nil. } \\
\text { Nil. } \\
\text { Nil. }\end{array}$ & $\begin{array}{ll} & \$ \\
3,200 & 00 \\
3,500 & 00 \\
3,333 & 33 \\
2,666 & 66 \\
5,625 & 00 \\
5,625 & 00 \\
3,999 & 94 \\
6,000 & 00 \\
& \\
6,000 & 00\end{array}$ \\
\hline & Total....... & 280 & 5,820 & Nil. & Nil. & \\
\hline
\end{tabular}

In 1918 this service, commencing at Halifax, was performed by the Provincial S.S. Co., and previous to that by the Halifax and Glace Bay S.S. Co.

\section{HALIFAX AND WEST COAST CAPE BRETON}

The Halifax axd Interness Steayship Co., Ltd.

Contract No. 70 .

T. \& C. File No. 25478.

Tote 174.-Halifax and West Coast of Cape Breton, calling at way ports, steam service between--

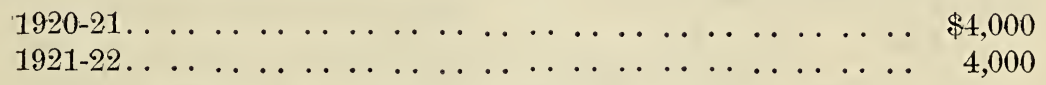

Contractors.-The Halifax and Inrerness SS. Co., Ltd., of Halifax, N.S.

Date of Contract.-April 13, 1920.

Duration of Contract.-From the opening to the close of navigation in 1920.

Service.-Weekly. Twenty-six trips shall constitute a full season's service, although the contractors shall be required to make such additional sailings as weather conditions shall permit.

Ports of Call.-Halifax, Port Mulgrave, Port Hawkesbury, Port Hastings, Port Hood, Mabou, Margaree, Grand Etang, Cheticamp, and Eastern Harbour, calling once every two weeks at Havre au Bouche, Cape George and Pleasant Bay, and calling at Inverness, Broadcove Marsh and Margaree Island whenever there is a reasonable quantity of freight or number of passengers to be landed or embarked, weather permitting.

- (b) Calls shall be made at Chimney Corner when the wharf is completed, and when there are passenger's or freight to be taken on or put off at that place.

(c) One call shall be made during the autumn of the present year at Ballantyne's Core, N.S.

(d) Through bills of lading shall be issued by the Strathlorne from Halifax to all ports in the Bras d'Or Lakes, and on the Eastern Coast of Cape Breton, called at by the Arcadia during the present season. 
(e) On all trips north the captain of the Strathlorne shall advise the agent of the contractors at Margaree from Mulgrave or Mabou the approximate hour of his arrival at Margaree, and on south bound trips he shall similarly advise the agent at Margaree from Grand Etang.

( $f$ ) When weather conditions at Margaree are such as to prevent boats or lighters coming out to the steamer, the agent shall hoist a red or white flag within a reasonable time of the steamer's announced arrival, so that the steamer may not be unduly delayed.

(g) On arrival off the port of Margaree the steamer shall anchor and give notice of her presence by whistle or other suitable means. If boats do not come off from the shore within half an hour after anchoring, the steamer shall be at liberty to hoist her anchor. and proceed on her voyage.

Government Wharves.-Steamer must call whenever possible.

Subsidy. $-\$ 4,000$ per annum, payable in instalments of $\$ 2,000$ on July 1 and on completion of the service.

Mails.-To be carried free.

\section{DISTANCES}

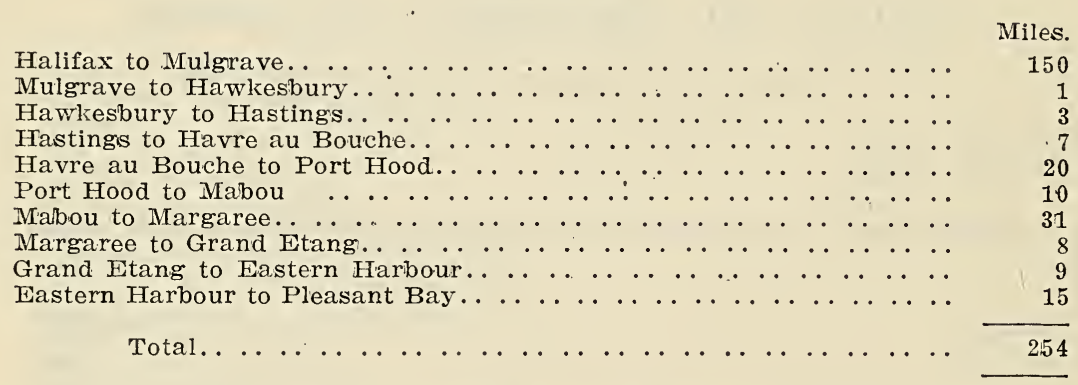

DESCRIPTION OF VESSEL EMPLOYED

\begin{tabular}{|c|c|c|c|c|c|c|c|c|c|c|c|c|}
\hline \multirow[b]{2}{*}{ Name. } & \multicolumn{3}{|c|}{ Dimensions. } & \multicolumn{3}{|c|}{ Tonnage. } & \multirow{2}{*}{ 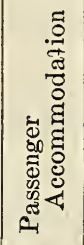 } & \multirow[b]{2}{*}{$\begin{array}{l}\dot{2} \\
\dot{4} \\
\dot{Z}\end{array}$} & \multirow[b]{2}{*}{ 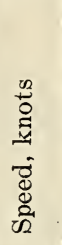 } & \multicolumn{3}{|c|}{ Built. } \\
\hline & 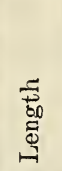 & 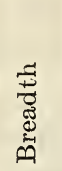 & 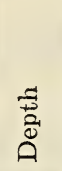 & 芯 & 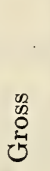 & 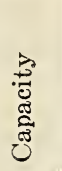 & & & & At & In & Of \\
\hline Strathlorne.. & $\begin{array}{l}\text { Ft. } \\
116\end{array}$ & $\begin{array}{l}\text { Ft. } \\
20 \cdot 2\end{array}$ & $\begin{array}{r}\text { Ft. } \\
9\end{array}$ & 81 & 135 & 180 & 40 & 24 & 9 & Mahone... & 1909 & Wood. \\
\hline
\end{tabular}


TRAFFIC RETURNS.

\begin{tabular}{|c|c|c|c|c|c|c|}
\hline Calendar Year. & $\begin{array}{c}\text { No. of round } \\
\text { trips run. }\end{array}$ & $\begin{array}{l}\text { No. of } \\
\text { Passengers } \\
\text { calried. }\end{array}$ & $\begin{array}{l}\text { Tons of } \\
\text { Freight } \\
\text { carried. }\end{array}$ & $\begin{array}{l}\text { Live } \\
\text { Stock. }\end{array}$ & $\begin{array}{l}\text { Bags } \\
\text { Mail. }\end{array}$ & $\begin{array}{c}\text { Subsidy } \\
\text { Paid. }\end{array}$ \\
\hline \multirow[t]{2}{*}{$\begin{array}{l}1912 . \\
1913 . \\
1914 . \\
1915 . \\
1916 . \\
1917 . \\
1918 . \\
1919 . \\
1920 .\end{array}$} & \multirow[t]{2}{*}{$\begin{array}{l}27 \\
27 \\
27 \\
27 \\
25 \\
27 \\
27 \\
27 \\
26\end{array}$} & $\begin{array}{lr} & 8 \\
& \text { Nil. } \\
& \text { Nil. } \\
& 3 \\
& \text { Nil. } \\
& 108 \\
& 64 \\
& 57 \\
\text { In } & 220 \\
\text { Out } & 250\end{array}$ & $\begin{array}{l}6,580 \\
5,120 \\
4,662 \\
3,782 \\
6,079 \\
7,150 \\
4,960 \\
3,125 \\
2,800 \\
3,200\end{array}$ & $\begin{array}{l}\text { Nil. } \\
\text { Nil. } \\
\text { Nil. } \\
\text { Nil. } \\
\text { Nil. } \\
\text { Nil. } \\
\text { Nil. } \\
\text { Nil. } \\
\text { Nil. } \\
\text { Nil. }\end{array}$ & $\begin{array}{l}\text { Nii. } \\
\text { Nii. } \\
\text { Nii. } \\
\text { Nii. } \\
\text { Nil. } \\
\text { Nii. } \\
\text { Nii. } \\
\text { Nii. } \\
\text { Nil. } \\
\text { Nil. }\end{array}$ & 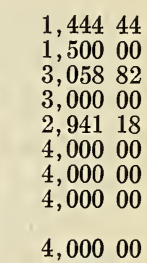 \\
\hline & & 470 & - 6,000 & Nil. & Nil. & \\
\hline
\end{tabular}

\section{MULGRAVE AND CANSO}

Hugh Cañ \& Son

Contract No. 53.

T. \& C. File No. 25837.

Vote 175.-Mulgrave and Canso, steam service between-

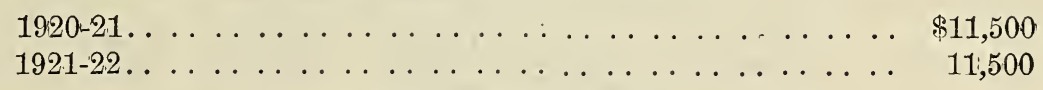

Contractors.-Hugh Cann \& Son, Ltd., of Yarmouth, N.S.

Date of Contract.-June 22, 1920.

Duration of Contract.-April 1, 1920, to March 31, 1921.

Service.-Daily (Sundays excepted).

Ports of Call.--Port Mulgrave and Canso, N.S.

Withdrawal for Repairs. - The steamer $R$. G. Cann may be withdrawn for repairs for one month in each year. During such withdrawal the service shall be performed by such steamer other than the Malcolm Cann or the Percy Cann as may be approved by the Minister.

Wind and Ice.-In the event of any trip or trips being missed on account of wind, fog, snow or ice upon satisfactory evidence being submitted to the Minister, he may direct that no deduction be made from the subsidy otherwise payable for the trip so missed.

Government Wharves.-Steamer must call whenever possible.

Subsidy.- $\$ 11,500$ per annum, payable in quarterly instalments in July, October, and January, and upon completion of the service.

(a) One-quarter of the subsidy otherwise payable shall be deducted when the contractors fail to make connections with the Intercolonial Railway at Mulgrave for both eastbound and westbound traffic in summer, and westbound traffic in winter, in sufficient time to permit of the transfer of passengers, mails and express shipments; but such penalty shall not be inflicted when the contractors are able to satisfy the Minister that the connection has been missed owing to wind, fog, snow or ice. 
11 GEORGE $\vee$, A. 1921

(b) $\$ 500$ of the subsidy shall be deducted should the contractors not place on the said route a substitute steamer other than the Malcolm Cann or the Percy Cann when the Robert G. Cann is taken off the said route for repairs as hereinbefore stipulated.

Mails.-To be carried free.

Distance.-Canso to Mulgrave, 24 miles.

DESCRIPTION OF VESSEL EMPLOYED.

\begin{tabular}{|c|c|c|c|c|c|c|c|c|c|c|c|c|}
\hline \multirow[b]{2}{*}{ Name. } & \multicolumn{3}{|c|}{ Dimensions. } & \multicolumn{3}{|c|}{ Tonnage. } & \multirow{2}{*}{ 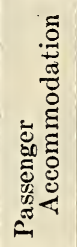 } & \multirow[b]{2}{*}{$\begin{array}{l}\dot{a} \\
\dot{z} \\
\dot{z}\end{array}$} & \multirow[b]{2}{*}{ 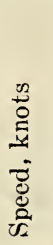 } & \multicolumn{3}{|c|}{ Built. } \\
\hline & 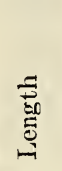 & 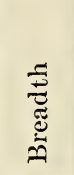 & $\frac{\overrightarrow{0}}{\stackrel{0}{0}}$ & 芩 & 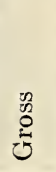 & 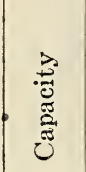 & & & & At & In & $\mathrm{O}$ \\
\hline $\begin{array}{r}\text { Robert G. } \\
\text { Cann..... }\end{array}$ & $\begin{array}{l}\text { Ft. } \\
119\end{array}$ & $\begin{array}{l}\text { Ft. } \\
24 \cdot 6\end{array}$ & $\begin{array}{l}\text { Ft. } \\
9 \cdot 4\end{array}$ & 111 & 265 & $\begin{array}{l}\text { Not } \\
\text { stated }\end{array}$ & 100 & 42 & 11 & Shelburne, N.S. & 1911 & Wood. \\
\hline
\end{tabular}

TRAFFIC RETURNS.

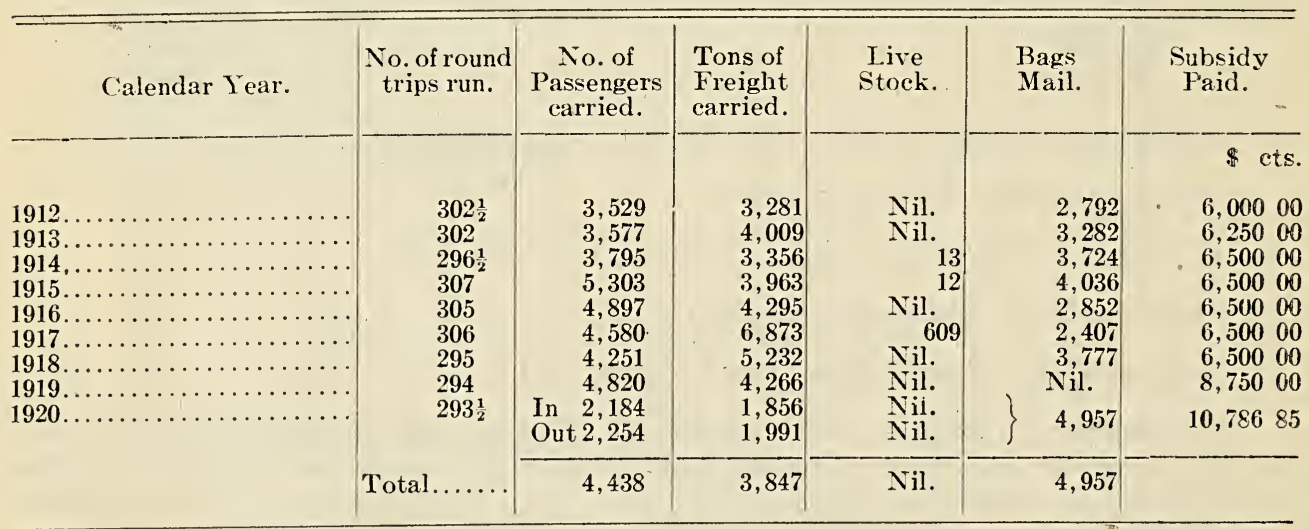

\section{MULGRAVE AND GUYSBOROUGH}

The Eraine Steamship Co., Lto.

Contract No. 54 .

T. \& C. File No. 25483.

Vote 1\%6.-Mulgrave and Guysborough, calling at intermediate ports, steam service between-

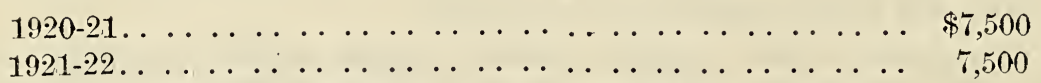

Contractors.-The Elaine Steamship Co., Itd., Halifax, N.S.

Date of Contract.-May 25, 1920.

Duration of Contract.-April 1, 1920, to March 31, 1921. 
SESSIONAL PAPER No. 10a

Service.-Daily (except Sundays).

Ports of Call.-Port Mifulgrave and Guysborough, calling at Queensport every trip from Guysborough to Mulgrave and three times each week on return trips to Guysborough on those days on which the steamer is not required to proceed to Boylston. Trips to Boylston shall be made three times each week, during the season of open navigation.

Repairs.-The steamer may be withdrawn one month during each year for repairs, etc. During such withdrawal the service shall be performed by such steamer as may be approved by the Minister.

Wind, Fog, Snow or Ice.-If any trip be missed on account of wind, fog, snow or ice, no deductions may be made from the subsidy otherwise payable for the trip so missed. April.

Subsidy.-\$7,500 per annum, payable quarterly, in July, October, January and

(a) One-quarter of the subsidy otherwise payable shall be deducted when the contractors fail to make connections with the Intercolonial Railway at Mulgrave for both eastbound and westbound traffic in the summer season and westbound traffic in the winter season in sufficient time to permit of the transfer of passengers, mails and express shipments, unless the connection has been missed owing to wind, fog, snow or ice.

Mails.-To be carried free.

\section{DISTANCES}

Miles.

$29 \frac{1}{3}$

sborough to Mulgrave (via Queensport) . . . . . . . . . .

Mulgrave to Guysiborough (direct) . . . . . . . . . . . . . .

Guysborough to Boylston...........................

DESCRIPTION OF VESSEL EMPLOYED.

\begin{tabular}{|c|c|c|c|c|c|c|c|c|c|c|c|c|}
\hline \multirow[b]{2}{*}{ Name. } & \multicolumn{3}{|c|}{ Dimensions. } & \multicolumn{3}{|c|}{ Tonnage. } & \multirow[b]{2}{*}{ 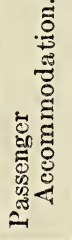 } & \multirow[b]{2}{*}{$\begin{array}{l}\dot{A} \\
\dot{1} \\
\dot{Z}\end{array}$} & \multirow[b]{2}{*}{ 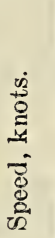 } & \multicolumn{3}{|c|}{ Built. } \\
\hline & 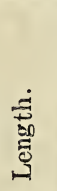 & 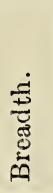 & $\begin{array}{c}\frac{\dot{A}}{3} \\
\frac{0}{0} \\
\text { A }\end{array}$ & $\begin{array}{l}\stackrel{+0}{0} \\
\text { Z }\end{array}$ & $\begin{array}{l}\dot{0} \\
0 \\
0 \\
0 \\
0\end{array}$ & 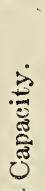 & & & & At & In & Of \\
\hline Westport III.. & $\begin{array}{l}\text { Ft. } \\
101\end{array}$ & $\begin{array}{l}\text { Ft. } \\
21\end{array}$ & $\begin{array}{c}\text { Ft. } \\
9\end{array}$ & 49 & 140 & 70 & 35 & 24 & 10 & Shelburne. & 1903 & Wood. \\
\hline
\end{tabular}

TRAFFIC RETURNS.

\begin{tabular}{l|r|r|r|r|r}
\hline & \\
&
\end{tabular}


11 GEORGE V, A. 1921

NEWCASTLE, NEGUAC AND ESCUMINAC, MIRAMICHI RIVER AND MIRAMICHI BAY

Miramichi Steam Navigation Company, Itdo.

Contract No. 49.

T. \& C. File 25665 .

Vote 17\%.-Newcastle, Neguac and Escuminac, calling at all intermediate points on the Miramichi River and Miramichi Bay, steam service between-

$$
\begin{aligned}
& 1920-21 \ldots \ldots \ldots \ldots \ldots \ldots \ldots \ldots \ldots \text {. . . . . . . . . . } \$ 3,000
\end{aligned}
$$

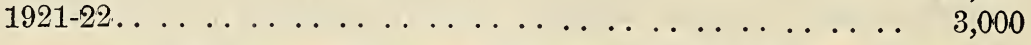

Contractors.-The Miramichi River Service, Ltd., of Chatham, N.B.

Date of Contract.-May 12, 1920.

Duration of Contract.-From the opening to the close of navigation in 1920 .

Service and Ports of Call.-On Mondays, Wednesdays and Fridays: From Newcastle, to and calling both ways at Chatham, Lower Newcastle, Loggieville, Oak Point, Church Point, Escuminac, Neguac and Mills Point Wharf.

On Tuesdays, Thursdays and Saturdays: From Newcastle, to and calling both ways at Chatham, Lower Newcastle, Loggieville, Oak Point, Baie du Vin, Church Point and Neguac.

The calls at Lower Newcastle and Mills Point Wharf are conditional upon the completion of the wharves at the respective points.

Calls at Escuminac are to be made during favourable weather in the salmonfishing season. When, owing to unfavourable weather, the steamer does not call at Escuminac, calls shall be made at Hardwick.

Government Wharves.-Steamer must call whenever possible.

Subsidy.- $\$ 3,000$, payable in two equal instalments, on September 1 and on the completion of the contract.

Mails.-To be carried free.

DISTANCES.

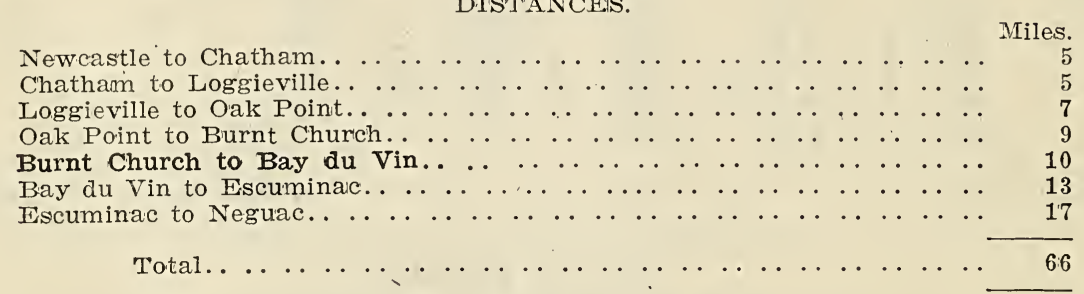

DESCRIPTION OF VESSEL EMPLOYED.

\begin{tabular}{|c|c|c|c|c|c|c|c|c|c|c|c|c|}
\hline \multirow[b]{2}{*}{ Name. } & \multicolumn{3}{|c|}{ Dimensions. } & \multicolumn{3}{|c|}{ Tonnage. } & \multirow{2}{*}{ 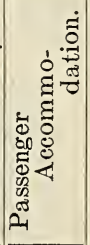 } & \multirow[b]{2}{*}{$\begin{array}{l}\dot{f} \\
\dot{u} \\
\dot{z}\end{array}$} & \multirow[b]{2}{*}{$\begin{array}{l}\dot{\Xi} \\
\Phi \\
\text { ळू. } \\
\text { की }\end{array}$} & \multicolumn{3}{|c|}{ Built. } \\
\hline & 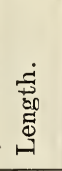 & 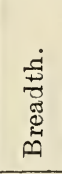 & 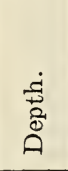 & 完 & $\begin{array}{l}\dot{v} \\
0 \\
0 \\
\dot{0} \\
0\end{array}$ & 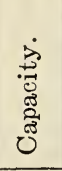 & & & & At & In & Of \\
\hline Alexandra... & $\begin{array}{r}\text { Ft. } \\
97\end{array}$ & $\begin{array}{l}\text { Ft. } \\
24.5\end{array}$ & $\begin{array}{r}\text { Ft. } \\
9\end{array}$ & 136 & $200^{\circ}$ & 50 & 400 & 38 & $\begin{array}{c}\text { Knots } \\
10\end{array}$ & Chatham. & 1902 & Wood. \\
\hline
\end{tabular}


TRAFFIC RETURNS.

\begin{tabular}{|c|c|c|c|c|c|c|}
\hline Calendar Year. & $\begin{array}{c}\text { No. of round } \\
\text { trips run. }\end{array}$ & $\begin{array}{c}\text { No. of } \\
\text { Passengers } \\
\text { carried. }\end{array}$ & $\begin{array}{l}\text { Barrels of } \\
\text { Freight } \\
\text { carried. }\end{array}$ & $\begin{array}{r}\text { Live } \\
\text { Stock. }\end{array}$ & $\begin{array}{l}\text { Mail } \\
\text { Bags. }\end{array}$ & $\begin{array}{l}\text { Subsidy } \\
\text { Paid. }\end{array}$ \\
\hline 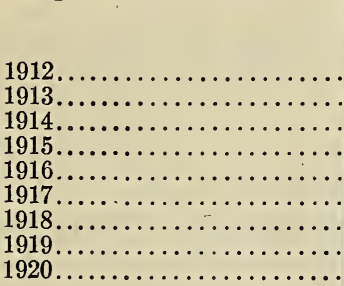 & $\begin{array}{l}175 \\
189 \\
160 \\
186 \\
180 \\
172 \\
177 \\
165 \\
153\end{array}$ & $\begin{array}{l}7,314 \\
8,228 \\
7,756 \\
7,762 \\
8,478 \\
7,848 \\
7,070 \\
8,278\end{array}$ & $\begin{array}{c}1,764 \\
1,960 \\
1,921 \\
2,404 \\
2,414 \\
2,275 \\
2,426 \\
2,558 \\
\text { No statisti }\end{array}$ & $\begin{array}{r}23 \\
56 \\
32 \\
37 \\
44 \\
36 \\
40 \\
24 \\
\text { savailable. }\end{array}$ & $\begin{array}{l}552 \\
557 \\
510 \\
594 \\
560 \\
544 \\
560 \\
508\end{array}$ & $\begin{array}{cc}\$ \\
2,500 & 00 \\
2,500 & 00 \\
2,262 & 00 \\
2,500 & 00 \\
2,500 & 00 \\
2,500 & 00 \\
2,500 & 00 \\
3,000 & 00 \\
3,000 & 00\end{array}$ \\
\hline
\end{tabular}

PELEE ISLAND AND THE MAINLAND

The Windsor and Pelee Island Steamship Co., Ltd.

Contract No. 52.

T. \& C. File 25417.

Vote 178.-Pelee Island and the Mainland, steam service between-

$$
\begin{array}{lllllllllllllllllll}
1920-21 & \ldots & \ldots & \ldots & \ldots & \ldots & \ldots & \ldots & \ldots & \ldots & \ldots & \ldots & \ldots & \ldots & \ldots & \ldots & \ldots & \$ 88,000 \\
1921-22 & \ldots & \ldots & \ldots & \ldots & \ldots & \ldots & \ldots & \ldots & \ldots & \ldots & \ldots & \ldots & \ldots & \ldots & \ldots & \ldots & \ldots & 8,000
\end{array}
$$

Contractors.-The Windsor and Pelee Island Steamship Co., Ltd., Scudder P.O., Pelee Island, Ont.

\section{Date of Contract.-April 1, 1920.}

Duration of Contract.-From the opening of navigation in 1920 to March 31, 1921.

Service and Ports of Call.-Six round trips each week, weather permitting, during the months of April, May, June, September and October, and five round trips each week during July, August and November, between Pelee Island and the mainland, calling on each trip at Kingsville and Leamington, Ont.

Tariff Rates.-Passenger rate for the trip between Pelee Island and Kingsville or Leamington or vice versa, $\$ 1$; and for the trip between Pelee Island and Windsor or vice versa, $\$ 1.50$. contract.

Freight rates are to be same as those contained in a schedule attached to the

Government Wharves.-Steamer must call whenever possible.

Subsidy. $-\$ 8,000$, payable in equal instalments on the last days of June, September. December and March.

Mails.-Are to be carried during open navigation between the post offices of Pelee Island, Pelee Island South and Scudider, and such post offices on the mainland as may be designated by the Postmaster General.

\section{DISTANCES.}

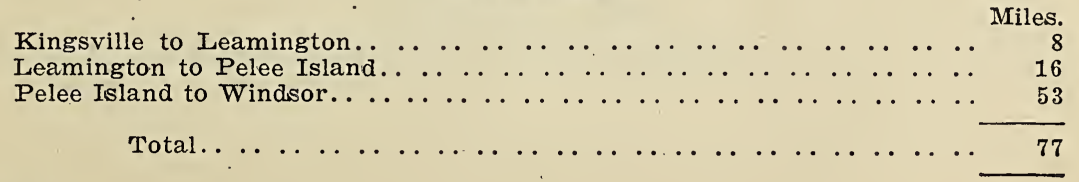

$10 \mathrm{a}-4$ 
11 GEORGE V, A. 1921

DESCRIPTION OF VESSEL EMPLOYED.

\begin{tabular}{|c|c|c|c|c|c|c|c|c|c|c|c|c|}
\hline \multirow[b]{2}{*}{ Name. } & \multicolumn{3}{|c|}{ Dimensions. } & \multicolumn{3}{|c|}{ Tonnage. } & \multirow{2}{*}{ 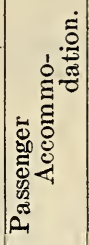 } & \multirow[b]{2}{*}{$\begin{array}{l}\dot{A} \\
\dot{z} \\
z\end{array}$} & \multirow[b]{2}{*}{$\begin{array}{l}\dot{8} \\
\stackrel{\Xi}{0} \\
\text { की }\end{array}$} & \multicolumn{3}{|c|}{ Built. } \\
\hline & 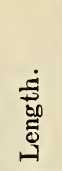 & 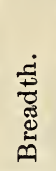 & $\begin{array}{l}\frac{5}{0} \\
\stackrel{0}{0} \\
0\end{array}$ & $\begin{array}{l}+0 \\
0 \\
z\end{array}$ & $\begin{array}{l}\dot{0} \\
\text { 总 } \\
\text { U⿺辶 }\end{array}$ & 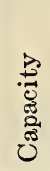 & & & & At & In & Of \\
\hline Pelee.. & $\begin{array}{l}\text { Ft. } \\
145\end{array}$ & $\begin{array}{r}\text { Ft. } \\
24\end{array}$ & $\begin{array}{r}\mathrm{Ft} . \\
13\end{array}$ & 242 & 537 & & 389 & 58 & $\begin{array}{r}\text { Knots } \\
13\end{array}$ & Collingwood. & 1914 & Steel. \\
\hline
\end{tabular}

TRAFFIC RETURNS.

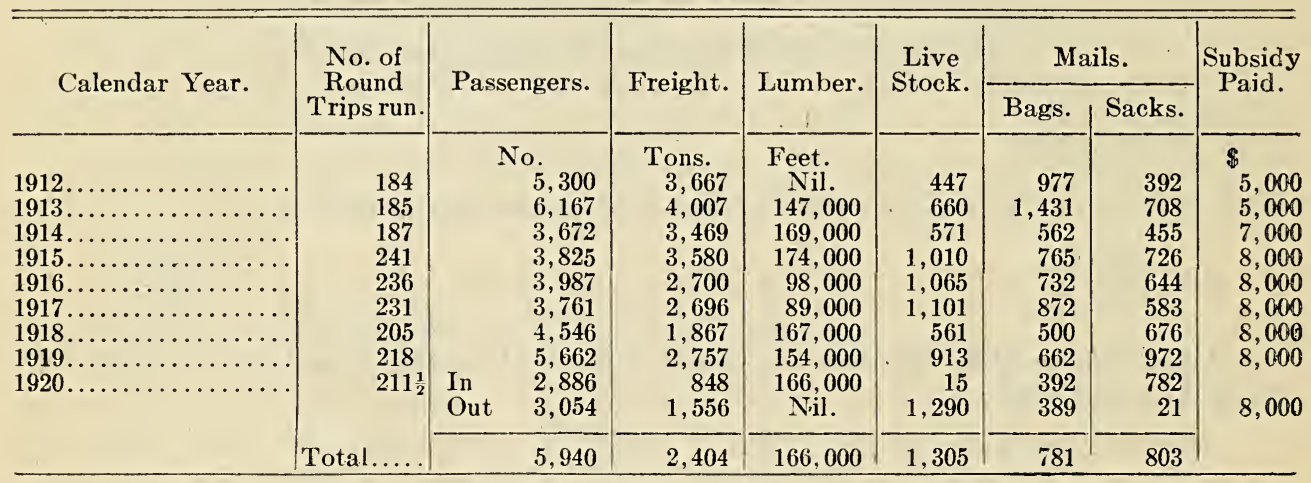


Grain and potatoes, per cwt., car lots

Grain and potatoes, less than carload...

Fruit in baskets, per cwt.

Wine, per bbl.

Oil, per barrel

Salt and flour, per bibl.

Minimum charge on one parcel.

Any one animal, horse or cattle up to five, each.

Any additionai animal over five.

Hogs and sheep up to ten, each...

Hogs and sheep over ten, each.

Groceries and hardware, per cw

Lumber, per $\mathrm{M}$ ft. up to $5 \mathrm{M} \mathrm{ft}$.

Over $5 \mathrm{M} \cdot \mathrm{tt}$.

Shingles, per $\ddot{M}$.

Laths, per M

,

Gristing, per bag, return

Tobacco, per cwt.

Buggies, set up, each....

Buggies, crated, each.

Coal, per ton...

Coal, per ton, in carload lots

Flour, per barrel.

Lubricating oils, per barrel.

Linseed oils, per barrel...

Turpentine, per barrel.

3 inch tile, per 100 .

$$
5 \text { “ }
$$$$
6 \text { “ }
$$$$
8
$$$$
10
$$$$
12
$$$$
\text { Fish, per cwt. }
$$$$
\begin{aligned}
& \text { "6 } \\
& \text { "6 } \\
& \text { " } \\
& \text { " }
\end{aligned}
$$

Automobiles, one way, including driver.

Automobiles, return, including driver.

Brick, per M...

Lime, per barrel.

Cement, per barrel

Cement, carloads.

㐌,

Beer, per case.

Pop, per case.

Eggs, per crate..

Hay and straw, per ton

Wool, per cwt.

Hides, per cwt.

Fence posts, each

Wagon, empty

Stone, per ton

Hardwall plaster, per cw

Scrap iron, per cwt

Cauliflower, per cwt

Pianos, each

Organs, each ........
Onions, in carloads

Onions, less than carloads.

Poultry, in crates, per cwt.

Poultry, not crated, per cwt

\begin{tabular}{|c|c|}
\hline $\begin{array}{c}\text { Kingsville } \\
\text { and } \\
\text { Leamington. }\end{array}$ & Windsor. \\
\hline$\$$ cts. & $\$$ cts. \\
\hline 010 & 012 \\
\hline $\begin{array}{ll}0 & 12\end{array}$ & 015 \\
\hline 030 & 030 \\
\hline 075 & 075 \\
\hline 085 & 085 \\
\hline 035 & 035 \\
\hline 025 & 025 \\
\hline 250 & 250 \\
\hline 225 & 225 \\
\hline 075 & 075 \\
\hline 065 & 065 \\
\hline 025 & 025 \\
\hline $\begin{array}{ll}350 \\
3 & 00\end{array}$ & $\begin{array}{ll}350 \\
3\end{array}$ \\
\hline 300 & 300 \\
\hline 040 & 040 \\
\hline 050 & 050 \\
\hline 020 & 020 \\
\hline 040 & $\begin{array}{lll}0 & 40\end{array}$ \\
\hline 175 & 175 \\
\hline 250 & 250 \\
\hline 200 & 200 \\
\hline 150 & 150 \\
\hline 045 & 045 \\
\hline 040 & 040 \\
\hline 085 & 085 \\
\hline 085 & 085 \\
\hline 075 & 075 \\
\hline 060 & 060 \\
\hline 080 & 080 \\
\hline 100 & 100 \\
\hline 120 & 120 \\
\hline 160 & 160 \\
\hline 200 & 200 \\
\hline 240 & 240 \\
\hline 035 & 035 \\
\hline 300 & 300 \\
\hline 500 & 500 \\
\hline 600 & 600 \\
\hline 050 & 050 \\
\hline 055 & 055 \\
\hline 050 & 050 \\
\hline 035 & 035 \\
\hline 025 & 025 \\
\hline 025 & 025 \\
\hline 250 & 250 \\
\hline 040 & $\begin{array}{ll}040 \\
0\end{array}$ \\
\hline 045 & 045 \\
\hline 005 & 005 \\
\hline 250 & 250 \\
\hline 150 & 150 \\
\hline 025 & 025 \\
\hline 025 & 025 \\
\hline $\begin{array}{ll}0 & 14\end{array}$ & $\begin{array}{ll}0 & 14\end{array}$ \\
\hline 500 & 500 \\
\hline 300 & 300 \\
\hline $\begin{array}{ll}0 & 10\end{array}$ & $\begin{array}{ll}0 & 12\end{array}$ \\
\hline 012 & 015 \\
\hline $\begin{array}{ll}0 & 75 \\
1 & 00\end{array}$ & $\begin{array}{ll}0 & 75 \\
1 & 00\end{array}$ \\
\hline 100 & \\
\hline
\end{tabular}

\section{PASSENGER FARES}

Between Pelee Island and Leamington and Kingsville-Single $\ldots \ldots \ldots \ldots \ldots \ldots \ldots 100$ 


\section{MULGRAVE, ARICHAT AND PETIT DE GRAT}

The LaHave Steamship Co., Ltd.

Contract No. 20.

T. \& C. File No. 25421.

Vote 179.-Mulgrave, Arichat and Petit de Grat, steam service between-

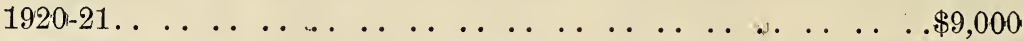

$$
\begin{aligned}
& 1921-22 \ldots \ldots \ldots \ldots \ldots \text {. . . . . . . . . . . . . . . . . . 9,000 }
\end{aligned}
$$

Contractor.-The LaHave Steamship Co., Ltd., of West LaHave, N.S.

Date of Contract.-April 3, 1920.

Duration of Contract.-April 1, 1920, to March 31, 1921.

Service and Ports of Call.-One full trip each way daily (Sundays excepted) between Petit de Grat and Mulgrave, calling on all trips both going and coming at Arichat and West Arichat.

Wind and Ice.-In the event of any trip or trips being missed on account of wind ex drifting ice, no deduction may be made from the subsidy otherwise payable for the rrips so missed.

Government Wharves. -Steamer must call whenever possible.

Subsidy. $\$ 9,000$, payable quarterly in July, October, January and April.

Repairs.-The steamer may be laid off during the month of August, 1920, for repairs, inspection, cleaning, painting, etc., and may also be laid off during the month of February, 1921, at the contractor's option. During the month of August, while the Tussle is laid off, the contractors shall run three round trips a week, with a substitute. vessel.

Mails. - To be carried free.

DISTANCES

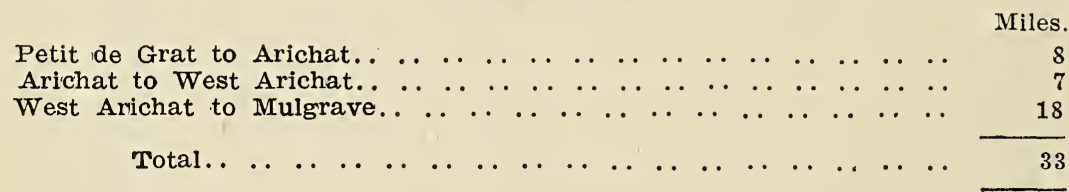

DESCRIPTION OF VESSEL EMPLOYED.

\begin{tabular}{|c|c|c|c|c|c|c|c|c|c|c|c|c|}
\hline \multirow[b]{2}{*}{ Name. } & \multicolumn{3}{|c|}{ Dimensions. } & \multicolumn{3}{|c|}{ Tonnage. } & \multirow{2}{*}{ 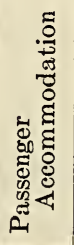 } & \multirow[b]{2}{*}{$\begin{array}{l}\dot{R} \\
\dot{y} \\
\dot{z}\end{array}$} & \multirow[b]{2}{*}{ 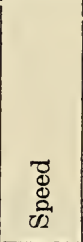 } & \multicolumn{3}{|c|}{ Built. } \\
\hline & 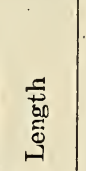 & 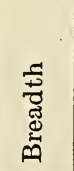 & 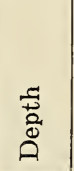 & 岕 & 资 & 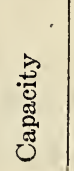 & & & & At & In & Of \\
\hline Tussle. & $\begin{array}{l}\text { Ft. } \\
83 \cdot 2\end{array}$ & $\begin{array}{l}\text { Ft. } \\
20.5\end{array}$ & $\begin{array}{c}\text { Ft. } \\
9 \cdot 8\end{array}$ & 67 & 151 & 100 & 25 & 28 & $\begin{array}{c}\text { knots } \\
10\end{array} \mid$ & Lunen burg, N.S. & 1915 & Wood \\
\hline
\end{tabular}


SESSIONAL PAPER No. 10a

TRAFFIC RETURNS

\begin{tabular}{|c|c|c|c|c|c|c|}
\hline Calendar Year. & $\begin{array}{c}\text { No. of round } \\
\text { trips run. }\end{array}$ & $\begin{array}{c}\text { No. of } \\
\text { Passengers } \\
\text { carried. }\end{array}$ & $\begin{array}{l}\text { Tons of } \\
\text { Freight } \\
\text { carried. }\end{array}$ & $\begin{array}{l}\text { Live } \\
\text { Stock. }\end{array}$ & $\begin{array}{c}\text { Mail } \\
\text { Sacks. }\end{array}$ & $\begin{array}{l}\text { Subsidy } \\
\text { Paid. }\end{array}$ \\
\hline 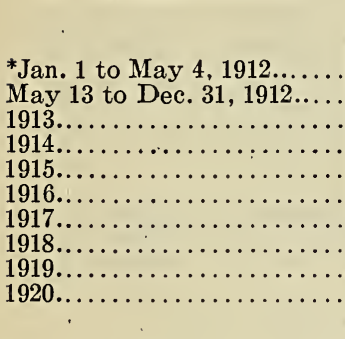 & $\begin{array}{l}96 \frac{1}{2} \\
190 \frac{1}{2} \\
281 \frac{1}{2} \\
276 \frac{1}{2} \\
275 \frac{1}{2} \\
241 \\
217 \frac{1}{2} \\
265 \\
242 \frac{1}{2} \\
166\end{array}$ & \begin{tabular}{|rr} 
& 747 \\
2,361 \\
3,160 \\
2,569 \\
2,140 \\
2,392 \\
1,535 \\
1,969 \\
2,108 \\
In \\
In 878 \\
Out..1, 1,508
\end{tabular} & $\begin{array}{r}285 \\
673 \\
1,070 \\
835 \\
832 \\
1,009 \\
1,106 \\
1,541 \\
2,051 \\
524 \\
351\end{array}$ & 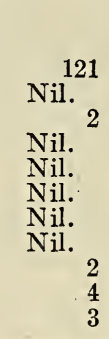 & $\begin{array}{r}236 \\
1,401 \\
2,672 \\
3,354 \\
2,944 \\
2,485 \\
2,235 \\
3,075 \\
3,503 \\
1,333 \\
. \quad 1,279\end{array}$ & $\begin{array}{rr}\$ \\
472 & 22 \\
3,149 & 04 \\
5,588 & 60 \\
6,750 & 00 \\
7,000 & 00 \\
6,125 & 00 \\
5,512 & 43 \\
7,000 & 00 \\
6,107 & 58 \\
7,562 & 50 \\
. & \end{array}$ \\
\hline Tota & & 2,386 & 875 & 7 & 2,612 & \\
\hline
\end{tabular}
N.S.

*From 1907 to May 4, 1912, the service was performed by Messrs. Hugh Cann \& Son, of Yarmouth;

\section{PICTOU AND MONTAGUE}

Georgetown S. S. Co., Ltd.

Contract No. 19.

T. \& C. File No. 25182.

Vote 180.-Pictou and Montague, calling at Murray Harbour and Georgetown, steam service between-

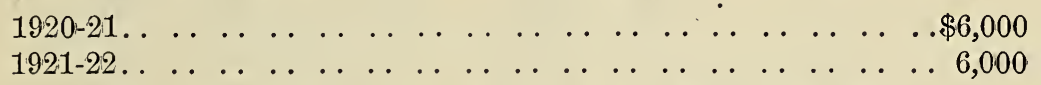

Contractor.-Georgetown Steamship Co., Ltd., of Pictou, N.S.

Date of Contract.-March 25, 1920.

Duration of Contract.-From the opening to the close of navigation in 1920.

Service and Ports of Call.-Two round trips each week (or three round trips each week, at contractor's option), sailing from Montague, calling at Georgetown and Beach Point, P.E.I., and Pictou, N.S., returning to Beach Point, Georgetown and Montague, and making one call each week at Cardigan, Newport and Murray Harbour North. Calls shall be made at Murray Harbour upon each trip, each way, at the minister's option, on evidence being produced that such calls are necessary.

Subsidy. $-\$ 6,000$ for the season, payable in three instalments.

Mails.-To be carried free.

Government Wharves.-Steamer must call whenever possible.

\section{DISTANCES}

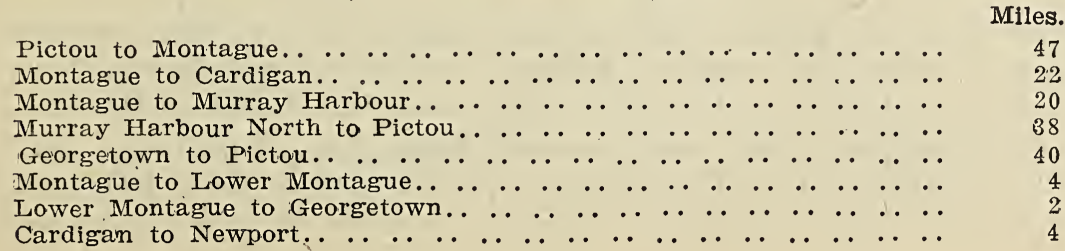


11 GEORGE V, A. 1921

DESCRIPTION OF VESSEL EMPLOYED.

\begin{tabular}{|c|c|c|c|c|c|c|c|c|c|c|c|c|}
\hline \multirow[b]{2}{*}{ Name. } & \multicolumn{3}{|c|}{ Dimensions. } & \multicolumn{3}{|c|}{ Tonnage. } & \multirow{2}{*}{ 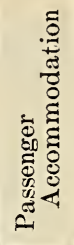 } & \multirow[b]{2}{*}{$\begin{array}{l}\dot{A} \\
\dot{4} \\
\dot{z}\end{array}$} & \multirow[b]{2}{*}{$\begin{array}{l}\overrightarrow{0} \\
\stackrel{0}{0} \\
\frac{\pi}{2}\end{array}$} & \multicolumn{3}{|c|}{ Built. } \\
\hline & 苟 & 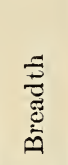 & $\begin{array}{l}\overrightarrow{\tilde{\Delta}} \\
\stackrel{\tilde{D}}{0}\end{array}$ & 芯 & 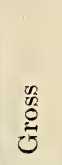 & 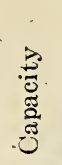 & & & & At & In & Of \\
\hline Magdalen... & $\begin{array}{l}\text { Ft. } \\
98 \cdot 6\end{array}$ & $\begin{array}{c}\text { Ft. } \\
21 \cdot 6\end{array}$ & $\begin{array}{l}\text { Ft. } \\
8 \cdot 8\end{array}$ & 91 & 134 & 150 & 18 & 28 & $\begin{array}{r}\text { knots } \\
10\end{array}$ & Shelburne, N.S. & $\begin{array}{l}1884 \\
\text { and } \\
1906\end{array}$ & Wood \\
\hline
\end{tabular}

TRAFFIC RETURNS

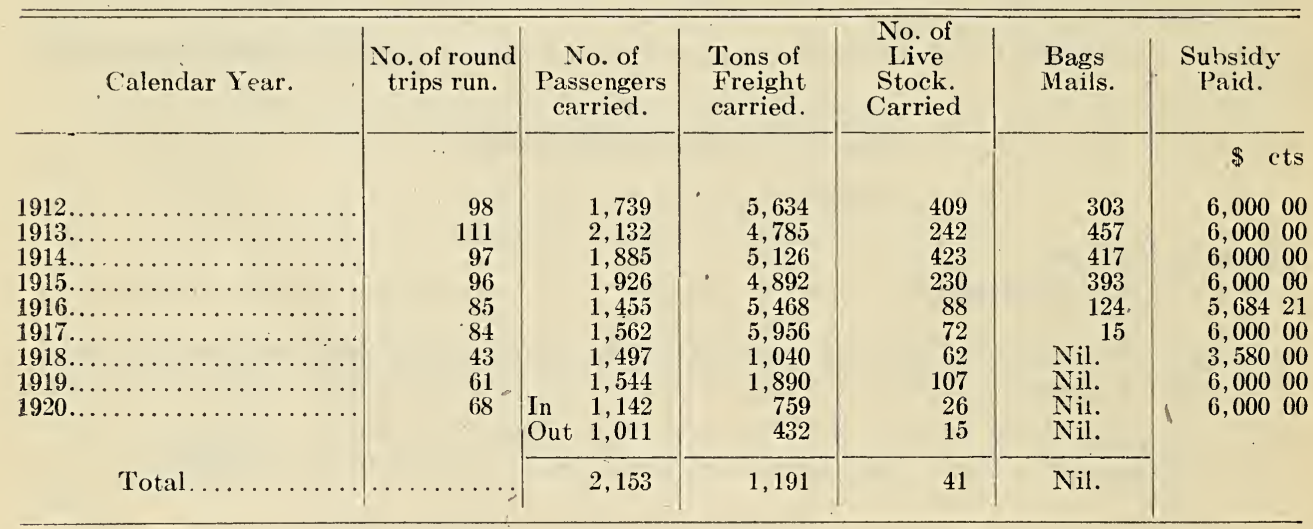

\section{PICTOL, MULGRAVE AND CHETICAMP}

RODERICK MCDOUgaLL

Contract No. 16.

T. \& C. File No. 25664.

Tote 181.-Pictou, Mulgrave and Cheticamp, steam service between-

$$
\begin{array}{rrrrrrrrrrrrrrrrr}
1920-21 . & \ldots & \ldots & \ldots & \ldots & \ldots & \ldots & \ldots & \ldots & \ldots & \ldots & \ldots & \ldots & \ldots & \ldots & \ldots & \$ 7,500 \\
1921-22 . & \ldots & \ldots & \ldots & \ldots & \ldots & \ldots & \ldots & \ldots & \ldots & \ldots & \ldots & \ldots & \ldots & \ldots & \ldots & 7,500
\end{array}
$$

Contractor:-Roderick McDougall, of Port Hood, N.S.

Date of Contract.- May 15, 1920.

Duration of Contract.-From the opening to the close of navigation, 1920.

Service and Ports of Call.--(a) 12 round trips during the season between Pictou and Mulgrave, calling both ways at Arisaig, Georgeville, Cape George, Morristown, Malignant Cove, Livingstone Cove, Ballantyne Cove and Cribbin's Point.

(b) Two round trips each week between Mulgrave and Cheticamp, calling both wavs at Port Hastings, Port Hawkesbury, Port Hood, Port Hood Island, Margaree Harbour, Grand Etang, and Eastern Harbour, making 50 round trips during the 
SESSIONAL PAPER No. 10a

season; and calling once each week at Pleasant Bay, and whenever passengers or freight are offered and whenever required by the minister at Henry Island, Mabou Mouth, Inverness, Red Cape and Port Bain, as well as at such other port or ports, place or places en route as the minister may direct.

(c) If so required by the minister eight round trips during the season between Souris and Mulgrave may be substituted for eight round trips between Pictou and Mulgrave aforesaid, calling both ways at Arisaig, Georgeville, Cape George, Morristown, Malignant Cove, Livingstone Cove, Ballantyne Cove and Cribbin's Point, of which one trip shall be made in June, two in July, two in August, one in September and two in October.

Regular semi-weekly connections are to be maintained with the Canadian National Railways at Mulgrave, and the Plant Line steamers at Hawkesbury.

Subsidy. $-\$ 7,500$ payable in equal instalments on the first days of July and October and on the completion of the service.

Mails.-To be carried free.

Government Wharves.-Steamer must call whenever possible.

DISTANCES

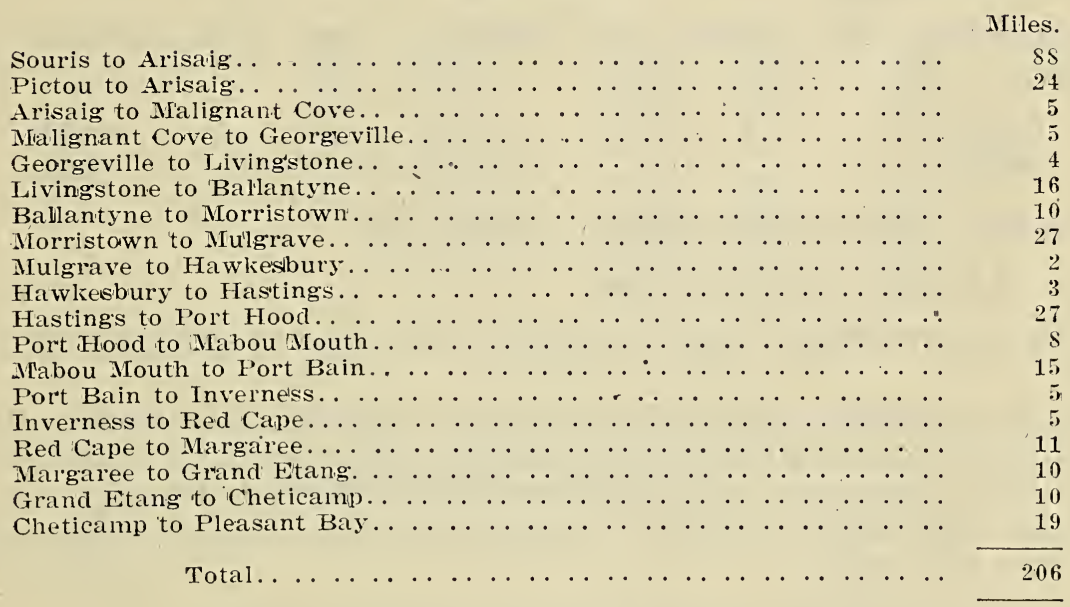

DESCRIPTION OF VESSEL EMPLOYED

\begin{tabular}{|c|c|c|c|c|c|c|c|c|c|c|c|c|}
\hline \multirow[b]{2}{*}{ Name. } & \multicolumn{3}{|c|}{ Dimension. } & \multicolumn{3}{|c|}{ Tonnage. } & \multirow{2}{*}{ 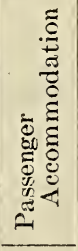 } & \multirow[b]{2}{*}{$\begin{array}{l}\dot{y} \\
\dot{z} \\
\dot{z}\end{array}$} & \multirow[b]{2}{*}{ 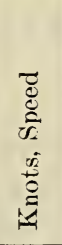 } & \multicolumn{3}{|c|}{ Built. } \\
\hline & 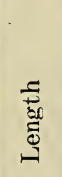 & 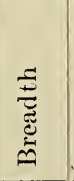 & $\stackrel{\overline{\vec{a}}}{\stackrel{0}{0}}$ & 苛 & 离 & 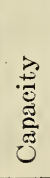 & & & & At & In & Of \\
\hline inburn. & $\begin{array}{l}\text { Ft. } \\
114\end{array}$ & $\begin{array}{l}\text { Ft. } \\
23 \cdot 5\end{array}$ & $\begin{array}{l}\text { Ft. } \\
10.5\end{array}$ & 79 & 168 & & 40 & 28 & 10 & $\begin{array}{l}\text { Mahone Bay, } \\
\text { N.s. }\end{array}$ & 1910 & Wood \\
\hline
\end{tabular}


TRAFFIC RETURNS.

\begin{tabular}{|c|c|c|c|c|c|c|c|}
\hline \multirow{2}{*}{ Calendar Year. } & \multirow{2}{*}{$\begin{array}{l}\text { No. of } \\
\text { Round } \\
\text { Trips } \\
\text { Run. }\end{array}$} & \multirow{2}{*}{$\begin{array}{l}\text { Number } \\
\text { of Passengers } \\
\text { carried. }\end{array}$} & \multicolumn{2}{|c|}{$\begin{array}{c}\text { Tons of Freight } \\
\text { carried. }\end{array}$} & \multirow{2}{*}{$\begin{array}{c}\text { Number } \\
\text { of } \\
\text { Live Stock. }\end{array}$} & \multirow{2}{*}{ Bags Mail. } & \multirow{2}{*}{ Subsidy Paid. } \\
\hline & & & Weight. & $\begin{array}{l}\text { Measure- } \\
\text { ment. }\end{array}$ & & & \\
\hline 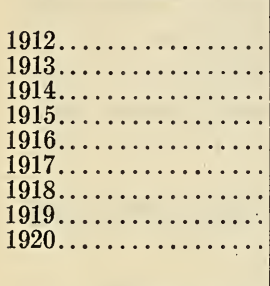 & $\begin{array}{l}86 \\
84 \\
64 \\
67 \\
66 \\
66 \\
62 \\
65 \\
65\end{array}$ & $\begin{array}{l}715 \\
904 \\
550 \\
315 \\
633 \\
343 \\
493 \\
824 \\
283 \\
171\end{array}$ & $\begin{array}{r}3,609 \\
2,687 \\
535 \\
1,145 \\
1,311 \\
922 \\
3,125 \\
9,000 \\
1,765 \\
680\end{array}$ & $\begin{array}{l}\text { Nil. } \\
\text { Nil. } \\
\text { Nil. } \\
\text { Nil. } \\
\text { Nil. } \\
\text { 545 } \\
\text { Nil. } \\
\text { Nil. } \\
\text { Nil. } \\
\text { Nil. }\end{array}$ & $\begin{array}{l}\quad 998 \\
257 \\
\text { Nil. } \\
\text { Nil. } \\
\text { Nil. } \\
\text { Nil. } \\
\text { Nil. } \\
\text { Nil. } \\
\text { Nil. } \\
\text { Nil. }\end{array}$ & $\begin{array}{l}\text { Nil. } \\
\text { Nil. } \\
\text { Nil. } \\
\text { Nil. } \\
\text { Nil. } \\
\text { Nil. } \\
\text { Nil. } \\
\text { Nil. } \\
\text { Nil. } \\
\text { Nil. }\end{array}$ & $\begin{array}{ll}\$ & \text { cts. } \\
6,718 & 75 \\
7,000 & 00 \\
5,942 & 70 \\
5,566 & 26 \\
8,106 & 39 \\
7,500 & 00 \\
7,500 & 00 \\
7,500 & 00 \\
7,500 & 00\end{array}$ \\
\hline Total.. & • & 454 & 2,445 & Nil. & Nil. & Nil. & \\
\hline
\end{tabular}

\section{PICTOU, NEW GLASGOW AND ANTIGONISH COUNTY PORTS}

\section{J. W. Sмiтн}

Contract No. 75.

T. \& C. File No. 25828.

Vote 182.-Pictou, New Glasgow and Antigonish County Ports, schooner service between-

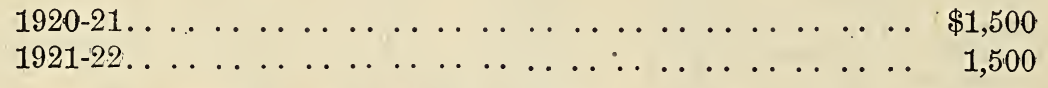

Contractor.-James Wilson Smith, of Pictou, N.S.

Date of Contract.-June 30, 1920.

Duration of Contract.-From the opening to the close of navigation (November 1), 1920.

Service and Ports of Call.-One round trip each week from Pictou, calling at New Glasgow, Lismore, McAra's Brook, Arisaig, Malignant Cove, Georgeville, Livingstone Cove, Ballantyne Cove, Morristown Wharf, Bayfield, Tracadie and Havre au Boucher. The call at Tracadie is contingent upon there being sufficient depth of water at the wharf there.

Government Wharves.-Steamer must call whenever possible.

Subsidy. $-\$ 1,500$, of which $\$ 750$ is payable on August 1 , and the balance upon the completion of the service.

Mails.-To be carried free.

\section{DISTANCES}

Miles.

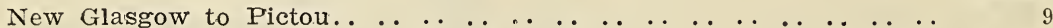

Pictou to Lismore..

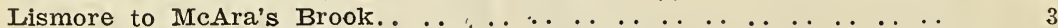

McAra's Brook to Arisaig.. . . . . . . . . . . . . . . . . . . . . . . . . . . . .

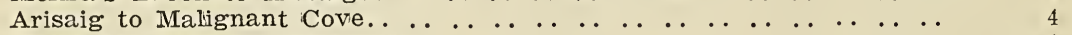

Malignant Cove to Georgeville. . . . . . . . . . . . . . . . . . . . . . .

Georgeville to Livingstone Cove.. . . . . . . . . . . . . . . . . . . . . . .

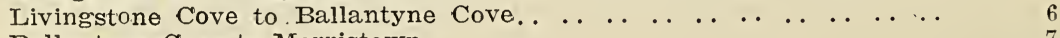

Ballantyne Cove to Morristown. . . . . . . . . . . . . . . . . . . . . . . .

$\begin{array}{lllllllllllllllllllll}\text { Total. . } & \ldots & \ldots & \ldots & \ldots & \ldots & \ldots & \ldots & \ldots & \ldots & \ldots & \ldots & \ldots & \ldots & \ldots & \ldots & \ldots & & 57\end{array}$ 
DESCRIPTION OF VESSEL EMPLOYED.

\begin{tabular}{|c|c|c|c|c|c|c|c|c|c|c|c|c|}
\hline \multirow[b]{2}{*}{ Name. } & \multicolumn{3}{|c|}{ Dimensions. } & \multicolumn{3}{|c|}{ Tonnage. } & \multirow{2}{*}{ 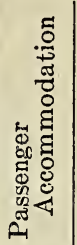 } & \multirow[b]{2}{*}{$\begin{array}{l}\dot{R} \\
\dot{4} \\
\dot{z}\end{array}$} & \multirow[b]{2}{*}{ 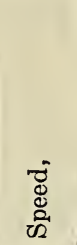 } & \multicolumn{3}{|c|}{ Built. } \\
\hline & 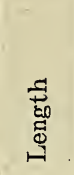 & 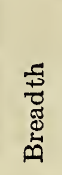 & 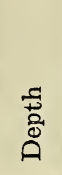 & $1 . \stackrel{0}{Z}$ & 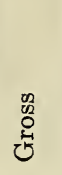 & 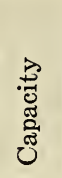 & & & & $\begin{array}{c}1 \\
\text { At }\end{array}$ & In & Of \\
\hline Ryșe... & $\begin{array}{l}\text { Ft. } \\
47 \cdot 3\end{array}$ & $\begin{array}{l}\text { Ft. } \\
12.8\end{array}$ & $\begin{array}{r}\text { Ft. } \\
6\end{array}$ & 20 & 20 . & 28 & Nil. & 30 & $\begin{array}{r}\text { knots } \\
8\end{array}$ & $\begin{array}{c}\text { Shippegan } \\
\text { Rebuilt } \\
\text { Souris.... }\end{array}$ & $\begin{array}{l}1889 \\
1914\end{array}$ & Wood \\
\hline
\end{tabular}

This vessel is a schooner, fitted with an auxiliary gasolene engine.

TRAFFIC RETURNS

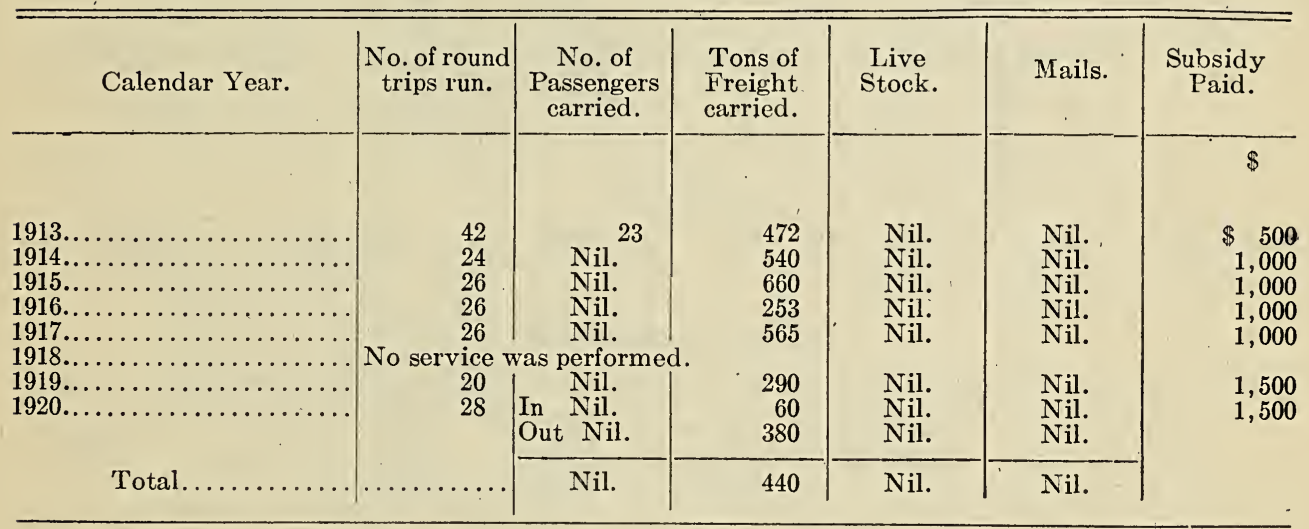

PORT MULGRAVE, ST. PETER'S, IRISH COVE AND MARBLE MOUNTAIN

Richmond Steamship Company

Contract No. 26.

T. \& C. File 25538.

Vote 183.-Port Mulgrave, St .Peter's, Irish Cove and Marble Mountain, and other ports on the Bras d'Or Lakes; steam service between-

$$
\begin{aligned}
& 1919-20 \ldots \ldots \ldots \ldots \ldots \ldots \ldots \ldots \ldots
\end{aligned}
$$

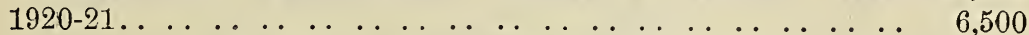

Contractors.-The Richmond Steamship Company, of Sydney, N.S.

Date of Contract.-April 22, 1920.

Duration of Contract.-From the opening to the close of navigation 1920.

Service and Ports of Call.-Two full round trips each week between Port Mulgrave, Poulamond, Grandique, River Bourgeois, St. Peter's, Johnston's Harbour, Irish Cove and Grand Narrows; and four full round trips each week between Grand Narrows and Marble Mountain, two of which each week are to extend to West Bay.

Government Wharves.-Steamer must call whenever possible. 
Subsidy. $-\$ 6,500$ per annum, payable in July, October, December and at the close of the service.

Mails.-To be carried free.

\section{DISTANCES}

Miles.

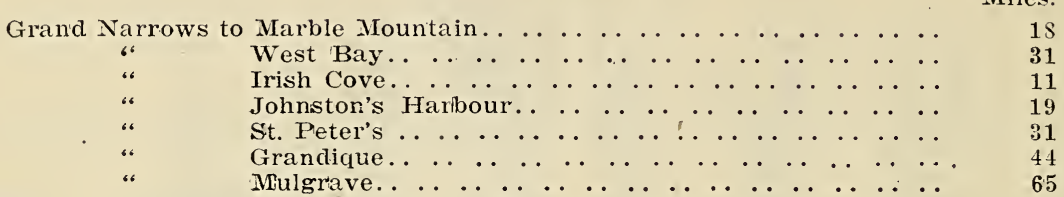

DESCRIPTION OF VESSEL EMPLOYED

\begin{tabular}{|c|c|c|c|c|c|c|c|c|c|c|c|c|}
\hline \multirow[b]{2}{*}{ Name. } & \multicolumn{3}{|c|}{ Dimensions. } & \multicolumn{3}{|c|}{ Tonnage. } & \multirow{2}{*}{ 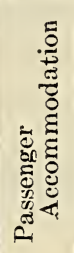 } & \multirow[b]{2}{*}{$\begin{array}{l}\dot{A} \\
\dot{r} \\
\dot{z}\end{array}$} & \multirow[b]{2}{*}{ 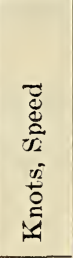 } & \multicolumn{3}{|c|}{ Built. } \\
\hline & 莺 & 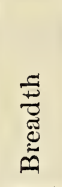 & 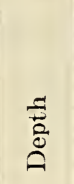 & $\begin{array}{l}\overrightarrow{0} \\
\ddot{z}\end{array}$ & 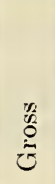 & 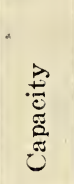 & & & & At & In & Of \\
\hline Pichmond.. & $\begin{array}{c}\text { Ft. } \\
112.5\end{array}$ & $\begin{array}{r}\text { Ft. } \\
18\end{array}$ & $\begin{array}{l}\text { Ft. } \\
8.25\end{array}$ & 105 & 162 & 75 & 100 & 21 & 9 & Sydney. & 1905 & Wood \\
\hline
\end{tabular}

TRAFFIC RETURNS

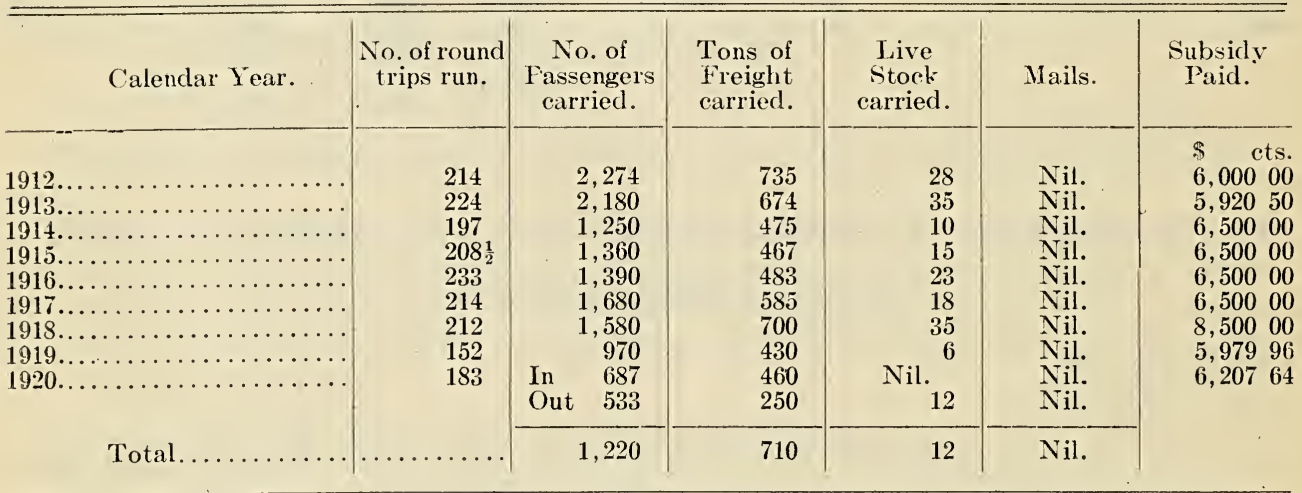

PICTOU, SOURIS AND THE MAGDALEN ISLANDS

WM. C. Leslie

Tote 184.-Pictou, Souris and the Magdalen Islands, steam service between-

$1920-21 \ldots \ldots \ldots$. . . . . . . . . . . . . See note below

$1921-22 \ldots \ldots \ldots \ldots \ldots \ldots \ldots \ldots 00$

(In 1920-21 this service was included in the vote of $\$ 70,000$, for services between Quebec and the North and South Shores of the Gulf of St. Lawrence, and between Pictou and the Magdalen Islands.)

Contractor.-Wm. C. Leslie. 
SESSIONAL PAPER NO. 10a

Date of Contract.-March 8, 1921.

Duration of Contract.-From the opening of navigation, 1921, to the close of navigation, 1925.

Service and Ports of Call.-A regular semi-weekly service between Pictou, Souris and the Magdalen Islands, as follows:-

(a) First trip each week: From Pictou, calling at Souris, Etang du Nord, Amherst, Grindstone and Pointe Bosse, returning to Grindstone, Amherst, Souris and Pictou.

(b) Second trip each week: From Pictou, calling at Souris, Amherst, Grindstone, Pointe Bosse and Grand Entry, returning to Pointe Bosse, Grindstone, Amherst, Souris and Pictou.

(c) Four trips each year are to be made, in place of four of the regular semiweekly trips aforesaid, from Pictou to Souris, West Point (to land Amherst mails), Etang du Nord, Bryan Island, Pointe Bosse, Grindstone, Amherst, Souris and Pictou.

Subsidy. $-\$ 24,000$ per annum, payable as follows: On July 1 and October 1 in each year, $\$ 8,000$; and on the close of narigation in each year, $\$ 8,000$.

Mails.-To be carried free.

\section{DISTANCES}

Mainland and Magdalen Island Service-

Pictou to Souris. . . . . . . . . . . . . . . . . . . . . . . . 5

Souris to Etang d'u Nord. . $\ldots \ldots \ldots \ldots$

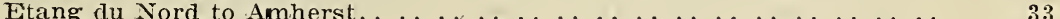

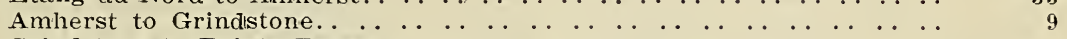

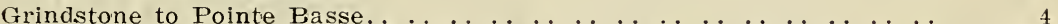

Pointe Basse to Grand Entry. . . . . . . . . . . . . . . . . . . . . . . . .

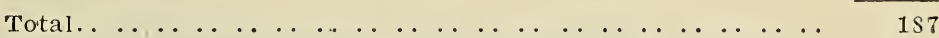

Etang du Nord to Bryan Island....................

DESCRIPTION OF VESSEL EMPLOYED.

\begin{tabular}{|c|c|c|c|c|c|c|c|c|c|c|c|c|c|}
\hline \multirow{2}{*}{ Name. } & \multicolumn{3}{|c|}{ Dimensions. } & \multicolumn{3}{|c|}{ ToNnage. } & \multirow{2}{*}{ 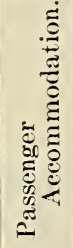 } & \multirow[b]{2}{*}{$\begin{array}{l}\dot{0} \\
\ddot{z}\end{array}$} & \multirow{2}{*}{ 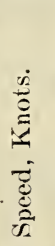 } & \multicolumn{4}{|c|}{ Built. } \\
\hline & 总 & 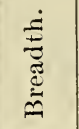 & $\frac{\stackrel{\vec{D}}{\overrightarrow{0}}}{\stackrel{0}{0}}$ & 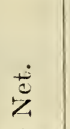 & $\underbrace{\dot{0}}_{\tilde{E}}$ & 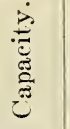 & & & & At & & In & Of \\
\hline R. W. Hendry & $\begin{array}{l}\text { Feet } \\
\text {. } 152\end{array}$ & $\begin{array}{r}\text { Feet } \\
29\end{array}$ & $\begin{array}{l}\text { Feet } \\
11 \frac{1}{2}\end{array}$ & 300 & 500 & 600 & $\begin{array}{c}30 \\
\text { and } \\
25\end{array}$ & $\ldots$ & 1 & $\begin{array}{l}2 \text { Gilbert's } \\
\text { X.S. }\end{array}$ & Cove, & 1921 & Wood. \\
\hline
\end{tabular}


TRAFFIC RETURNS.

\begin{tabular}{|c|c|c|c|c|c|c|c|}
\hline \multirow{2}{*}{$\begin{array}{l}\text { Calendar } \\
\text { Year. }\end{array}$} & \multirow{2}{*}{$\begin{array}{c}\text { No. of } \\
\text { Round } \\
\text { Trips run. }\end{array}$} & \multirow{2}{*}{$\begin{array}{c}\text { Number of } \\
\text { Passengers } \\
\text { carried. }\end{array}$} & \multirow{2}{*}{$\begin{array}{l}\text { Tons of } \\
\text { Freight } \\
\text { carried. }\end{array}$} & \multirow[t]{2}{*}{ Live Stock. } & \multicolumn{2}{|c|}{ Mails. } & \multirow{2}{*}{$\begin{array}{c}\text { Subsidy } \\
\text { Paid. }\end{array}$} \\
\hline & & & & & Lock Bags. & Tied Sacks. & \\
\hline $\begin{array}{l}1907 \ldots \ldots \ldots \\
1908 \ldots \ldots \ldots \\
1909 \ldots \ldots \ldots \\
1910 \ldots \ldots \\
1911 \ldots \ldots \ldots \\
1912 \ldots \ldots \ldots \\
1913 \ldots \ldots \ldots \\
1914 \ldots \ldots \ldots \\
1915 \ldots \ldots \ldots \\
1916 \ldots \ldots \ldots \\
1917 \ldots \ldots \ldots \\
1918 \ldots \ldots \ldots \\
1919 \ldots \ldots \ldots \\
1920 \ldots \ldots \ldots\end{array}$ & $\begin{array}{l}58 \\
61 \\
62 \\
70 \\
61 \\
64 \\
68 \\
61 \\
60 \\
52 \\
54 \\
43 \\
53 \\
54 \frac{1}{2}\end{array}$ & \begin{tabular}{|lr}
880 \\
1,357 \\
1,168 \\
1,394 \\
1,416 \\
1,109 \\
642 \\
1,380 \\
633 \\
1,714 \\
1,913 \\
1,379 \\
1,651 \\
In $\quad 822$ \\
Out 1,130
\end{tabular} & $\begin{array}{l}5,900 \\
4,472 \\
1,419 \\
6,048 \\
6,173 \\
7,433 \\
6,905 \\
4,802 \\
8,462 \\
6,591 \\
8,860 \\
4,932 \\
9,500 \\
1,870 \\
4,110\end{array}$ & $\begin{array}{r}\text { Not given. } \\
18 \\
944 \\
1,109 \\
1,269 \\
508 \\
727 \\
811 \\
174 \\
26 \\
69 \\
130 \\
119 \\
40 \\
129\end{array}$ & 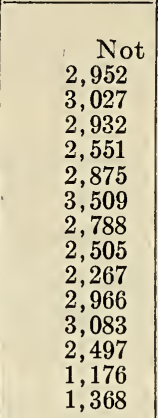 & $\begin{array}{r}\text { given. } \\
\text { Nil. } \\
169 \\
340 \\
308 \\
880 \\
482 \\
921 \\
1,297 \\
1,239 \\
505 \\
1,393 \\
1,469 \\
1,018 \\
48\end{array}$ & $\begin{array}{rr}\$ & \text { cts. } \\
15,000 & 00 \\
15,000 & 00 \\
12,000 & 00 \\
15,000 & 00 \\
15,000 & 00 \\
14,807 & 70 \\
15,000 & 00 \\
18,000 & 00 \\
17,769 & 23 \\
14,000 & 00 \\
17,769 & 23 \\
18,000 & 00 \\
18,000 & 00\end{array}$ \\
\hline 1920. & Total... & 1,952 & 5,980 & 169 & 2,544 & 1,066 & $16,934 \quad 45$ \\
\hline
\end{tabular}

The steamer Scotsburn was also chartered from Hendry, Ltd., for one single trip, commencing on December 18, 1920, for $\$ 2,400$. She carried 245 tons of freight. The Stanley also made two trips.

From 1909 to 1915 inclusive this service was performed by Wm. McLure, of Pictou, N.S.; in 1916 and 1917 by the Magdalen Islands SS. Co., Ltd.; in 1918 by J. A. Farquhar \& Co., Ltd., of Halifax; and in 1919 and 1920 by the Gulf of St. Lawrence Shipping and Trading Co., of Quebec.

\section{QUEBEC, NATASHQUAN AND HARRINGTON}

Clarke Bros.

Vote 185.-Quebec, Natashquan and Harrington, and other points on the North Shore of the Gulf of St. Lawrence, steam service between-

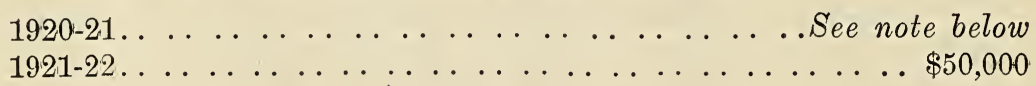

\section{QUEBEC, MONTREAL AND PASPEBIAC}

Clarke Bros.

Vote 186.-Quebec, Montreal and Paspebiac, and ports on the South Shore of the Gulf of St. Lawrence, steam service between-

$$
\begin{aligned}
& \text { 1920-21. . . . . . . . . . . . . . . . See note below }
\end{aligned}
$$

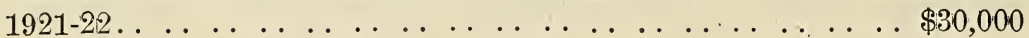

(In 1920-21 these two services were included in the vote of $\$ 70,000$ for steam services on the North and South Shores of the Gulf of St. Lawrence, etc.)

The two preceding services (Votes 185 and 186) are covered by one contract.

Contractors.-Clarke Bros., Quebec, Que.

Date of Contract.-February 28, 1921. 
SESSIONAL PAPER No. 10a

Duration of Contract.-From the opening of navigation in 1921 to the close of navigation in 1925 .

\section{QUEBEC, NATASIRQUAN AND HARRINGTON SERVICE}

Service and Ports of Call.-Three round trips each month from Quebec to Natashquan, calling each way at Godbout, Franquelin River, Trinity Bay, Egg Island, Pentecost, Clarke City, Seven Islands, Rivière aux Graines, Sheldrake, Thunder River, Magpie, St. John River, Longue Pointe, Mingan, Esquimaux Point, Piastre Bay and Natashquan, extending one trip each month to Harrington.

Subsidy. $-\$ 50,000$ per annum, payable on June 1, August 1, October 1, and at the close of navigation in each year.

Mails.-To be carried free.

\section{DISTANCES}

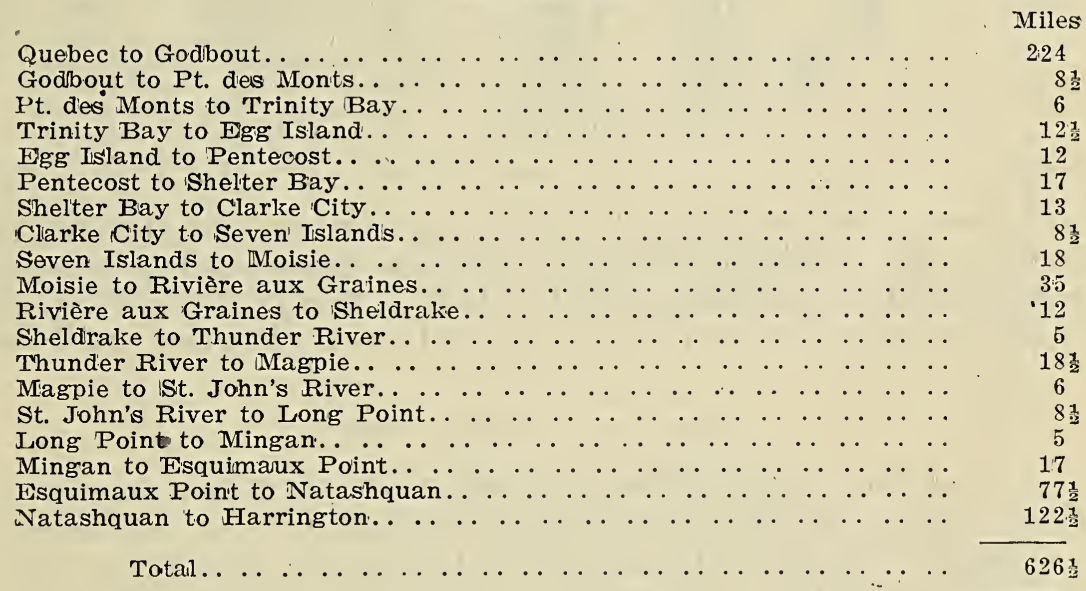

DESCRIPTION OF VESSEL EMPLOYED.

\begin{tabular}{|c|c|c|c|c|c|c|c|c|c|c|c|c|}
\hline \multirow{2}{*}{ Name. } & \multicolumn{3}{|c|}{ Dimensions. } & \multicolumn{3}{|c|}{ Tonnage. } & \multirow{2}{*}{ 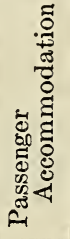 } & \multirow[b]{2}{*}{$\begin{array}{l}\dot{B} \\
\dot{H} \\
\dot{z}\end{array}$} & \multirow{2}{*}{ 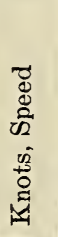 } & \multicolumn{3}{|c|}{ Built. } \\
\hline & 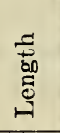 & 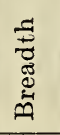 & 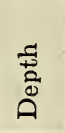 & $\overrightarrow{0}$ & 品 & 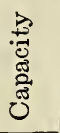 & & & & At & In. & Of. \\
\hline North Shore. & $\begin{array}{l}\text { Ft. } \\
206\end{array}$ & $\begin{array}{l}\text { Ft. } \\
28 \frac{1}{2}\end{array}$ & $\begin{array}{r}\text { Ft. } \\
13\end{array}$ & 480 & 811 & 500 & $\begin{array}{c}50 \\
\text { and } \\
50\end{array}$ & 122 & $11 \frac{1}{2}$ & Newcastle. & 1896 & Steel. \\
\hline
\end{tabular}


TRAFFIC RETURNS.

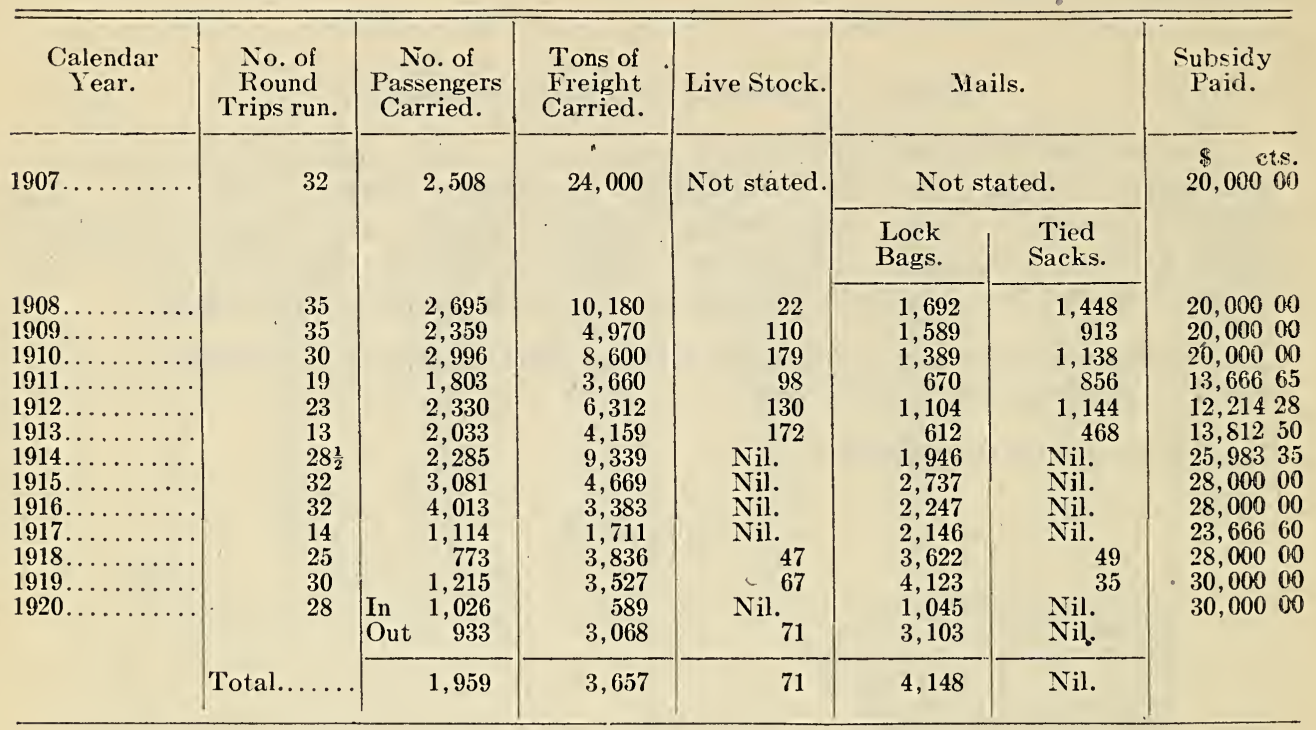

From 1908 to 1913 inclusive the service was performed by Holliday Bros., of Quebec; from 1914 to 1916 inclusive by the Canada Steamship Lines, Ltd.; in 1917 by. the Gaspé and Baie des Chaleurs SS. Co., Ltd.; in 1918 by G. F .Gibsone and J. E. Bernier, and in 1919 and 1920 by the Gulf of St. Lawrence Shipping and Trading Co.

QUEBEC AND PASPEBIAC SERVICE

Service and Ports of Call.-Two round trips each month from Quebec (or Montreal, at the contractor's option) to Paspebiac, ealling each way at Cap Chat, Ste Anne des Monts, Mont Louis, Grande Vallée, Chlorydomes, Fox River, Griffin Cove, Anse à Louise, Grand Grève, Douglastown, Gaspé Basin, Malbaie, Percé, Cape Cove, Grand River, Chandler, Newport and Port Daniel; and calling once each rovage, either on up or down trip, at Mechins, Marsouins, Martin River, Claude River, St. Antoine, Magdalen, Petite Vallée, Pointe Fregate, St. Yron (Pointe'Sèche), Grand Etang, Anse à Valeau and Pointe Jaune, subject to favourable weather and water conditions.

At the discretion of the minister; the contractors may be permitted to omit from time to time such calls at the above ports as may be deemed advisable, or to call alternately or otherwise at such ports as may be required by the minister, having in view the public interest, and the efficient despatch of passengers and freight.

Subsidy.- $\$ 30,000$ per annum, payable on June 1, August 1, October 1 , and on the close of navigation in each year.

Mails.-To be carried free. 
SESSIONAL PAPER No. 10a

\section{DISTANCES}

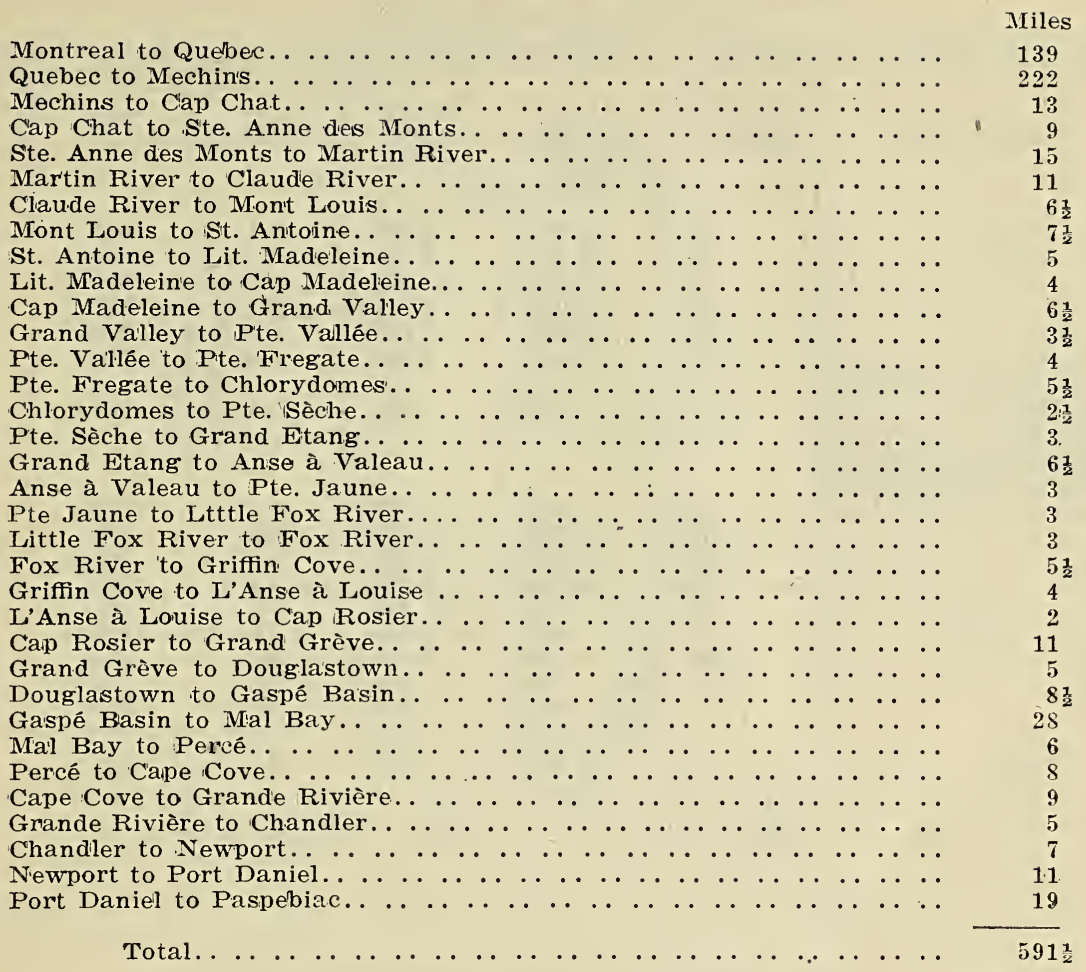

DESCRIPTION OF VESSEL EMPLOYED.

\begin{tabular}{|c|c|c|c|c|c|c|c|c|c|c|c|c|}
\hline \multirow[b]{2}{*}{ Name. } & \multicolumn{3}{|c|}{ Dimensions. } & \multicolumn{3}{|c|}{ Tonnage. } & \multirow{2}{*}{ 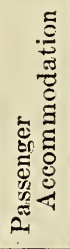 } & \multirow[b]{2}{*}{$\begin{array}{l}\dot{a} \\
\dot{z} \\
\dot{z}\end{array}$} & \multirow[b]{2}{*}{ 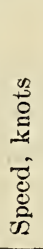 } & \multicolumn{3}{|c|}{ Built. } \\
\hline & 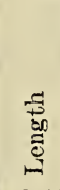 & 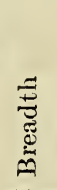 & & $\stackrel{\vec{c}}{z}$ & 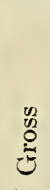 & 总 & & & & At & In & Of \\
\hline & Ft. & Ft. & Ft. & & & & & & & & & \\
\hline
\end{tabular}




\section{TRAFFIC RETURNS}

SOUTH SHORE SERVICE

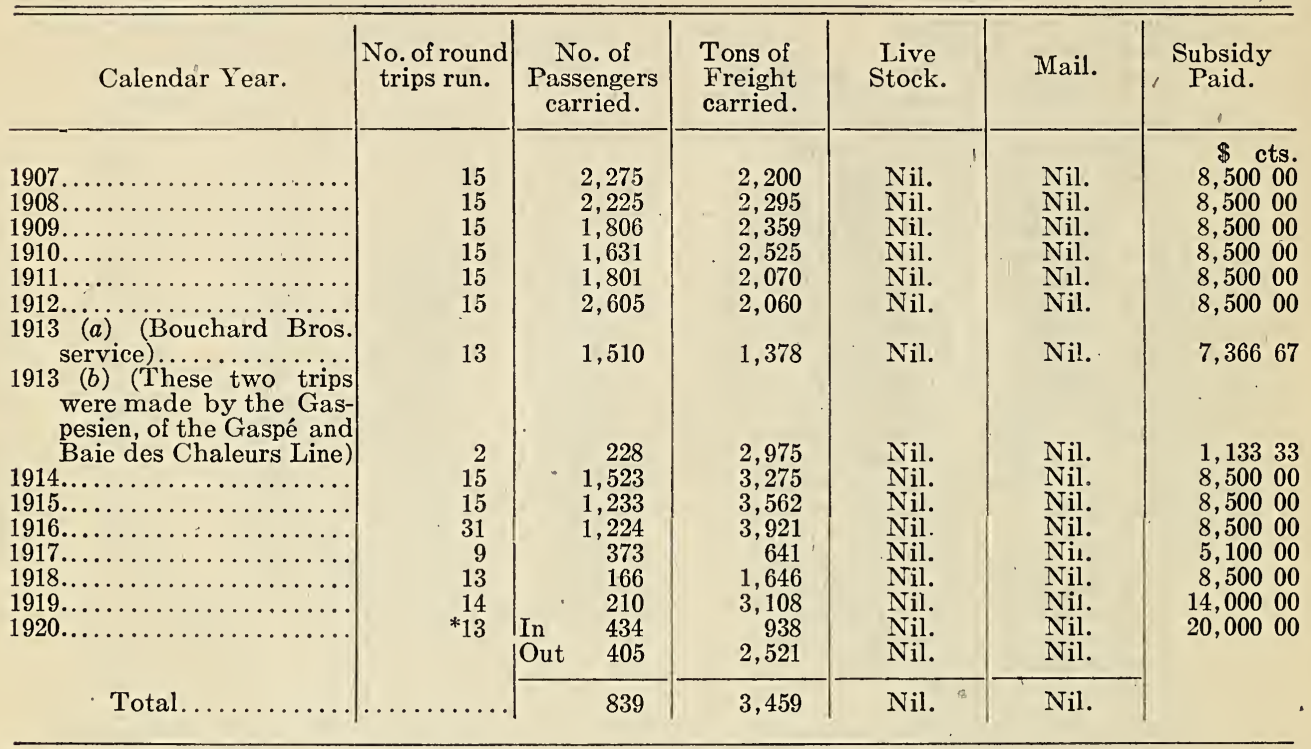

* 11 trips were also performed by the "A. Tremblay," without subsidy.

\section{ST. JOHN AND ST. ANDREWS, N.B.}

Maritime Steamship Company, Ltd.

Contract No. 41.

T. \& C. File No. 25744.

Vote 18\%.-St. John, N.B., and St. Andrews, N.B., calling at intermediate points, steam service between-

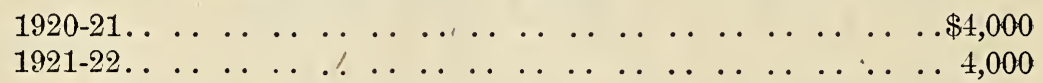

Contractors.-The Maritime Steamship Company, Ltd., of Black's Harbour, N.B.

Date of Contract.-June 5, 1920.

Duration of Contract.-April 1, 1920, to March 31, 1921.

Service and Ports of Call.-A regular service between St. John and St. Andrews, calling at Dipper Harbour, Black's Harbour, Back Bay, Letete, Deer Island, and St. George.

One round trip per week is to be run throughout the year, as follows:-

(a) Leave St. John for St. Andrews on Saturday mornings, calling at Dipper Harbour, Beaver Harbour, Black's Harbour, Back Bay or Letete, Deer Island and Red 'Store or St. George.

(b) Returning leave St. Andrews for St. John on Tuesday mornings, calling at Letete or Back Bay, Black's Harbour, Beaver Harbour and Dipper Harbour. 
SESSIONAL PAPER No. 10a

Government Wharves.-Steamer must call whenever possible.

Subsidy. - $\$ 4,000$ per annum, payable as follows: On October $1, \$ 2,000$ and on the completion of the service, $\$ 2,000$.

Mails.-To be carried free.

\section{DISTANICES}

Miles.

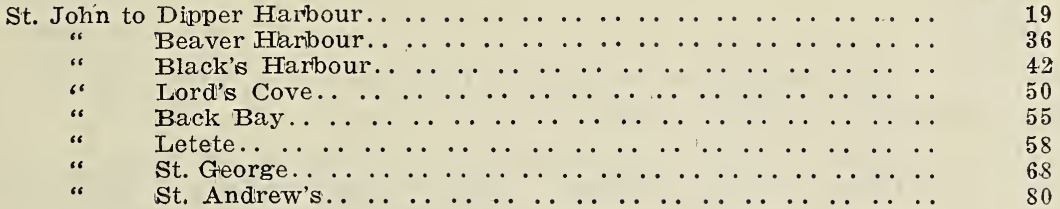

DESCRIPTION OF VESSEL EMPLOYED

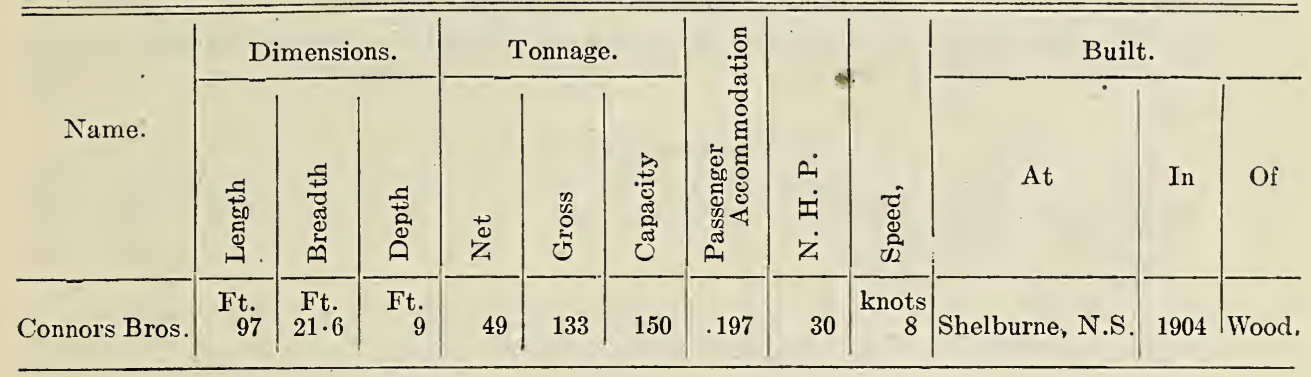

TRAFFIC RETURNS

\begin{tabular}{|c|c|c|c|c|c|c|c|}
\hline \multirow{2}{*}{$\begin{array}{l}\text { Calendar } \\
\text { Year. }\end{array}$} & \multirow{2}{*}{$\begin{array}{c}\text { No. of } \\
\text { Round } \\
\text { Trips run. }\end{array}$} & \multirow{2}{*}{$\begin{array}{l}\text { Number of } \\
\text { Passengers } \\
\text { carried. }\end{array}$} & \multirow{2}{*}{$\begin{array}{l}\text { Tons of } \\
\text { Freight } \\
\text { carried. }\end{array}$} & \multirow{2}{*}{ Live Stock } & \multicolumn{2}{|c|}{ Mails. } & \multirow{2}{*}{$\begin{array}{l}\text { Subsidy } \\
\text { Paid. }\end{array}$} \\
\hline & & & & & Lock Bags. & Tied Sacks. & \\
\hline \multirow[t]{2}{*}{$\begin{array}{l}1912 \ldots \ldots \ldots \\
1913 \ldots \ldots \ldots \\
1914 \ldots \ldots \\
1915 \ldots \ldots \\
1916 \ldots \ldots \\
1917 \ldots \ldots \\
1918 \ldots \ldots \\
1919 \ldots \ldots \\
1920 \ldots \ldots \\
\end{array}$} & $\begin{array}{l}48 \\
47 \\
48 \\
50 \\
52 \\
52 \\
52 \\
48 \\
53\end{array}$ & $\begin{array}{r}1,095 \\
1,091 \\
1,121 \\
1,276 \\
1,275 \\
1,289 \\
1,280 \\
1,630 \\
\text { In } \quad 472 \\
\text { Out } \quad 608\end{array}$ & $\begin{array}{l}4,825 \\
4,656 \\
5,436 \\
5,884 \\
6,846 \\
5,719 \\
7,039 \\
9,169 \\
2,109 \\
3,651\end{array}$ & $\begin{array}{l}\text { Nil. } \\
\text { Nil. } \\
\text { Nil. } \\
\text { Nil. } \\
\text { Nil. } \\
\text { Nil. } \\
\text { Nil. } \\
\text { Nil. } \\
\text { Nil. } \\
\text { Nil. }\end{array}$ & $\begin{array}{l}192 \\
188 \\
200 \\
200 \\
208 \\
184 \\
498 \\
418 \\
192 \\
233\end{array}$ & $\begin{array}{l}\text { Nil. } \\
\text { Nil. } \\
\text { Nil. } \\
6 \\
12 \\
22 \\
12 \\
12 \\
4 \\
44\end{array}$ & $\begin{array}{lr}\$ & \text { cts. } \\
3,769 & 23 \\
4,000 & 00 \\
4,000 & 00 \\
4,000 & 00 \\
4,000 & 00 \\
4,000 & 00 \\
4,000 & 00 \\
4,000 & 00 \\
4,000 & 00\end{array}$ \\
\hline & Total....... & 1,080 & 5,760 & Nil. & 425 & 48 & \\
\hline
\end{tabular}




\section{ST. JOHN AND BRIDGETOWN}

Bribgetown Steamship Company

Contract No. 72 .

T. \& C. File No. 25682.

Vote 188.-St. John and Bridgetown, steam service between-

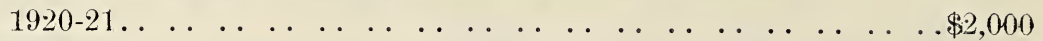

$$
\begin{aligned}
& 1921-22 \ldots \ldots \ldots \ldots \ldots \ldots
\end{aligned}
$$

Contractors.-The Bridgetown Steamship Company, Ltd., of Bridgetown, N.S.

Date of Contract.-May 21, 1920.

Duration of Contract.-From the opening to the close of navigation in 1920.

Service and Ports of Call.-Weekly; making thirty-two round trips during the season of navigation between St. John and Bridgetown, calling each way at Ray's Creek, Tupperville and Round Hill; and calling fortnightly at Ciementsport and Deep Brook.

Government Wharves.-Steamer must call whenever possible.

Subsidy. $\$ 62.50$ for each round trip, not to exceed a total of $\$ 2,000$ for the season, payable on 'July 1, Oetober 1, and at close of navigation.

Mails.-To be carried free.

\section{DISTANCES}

Miles.

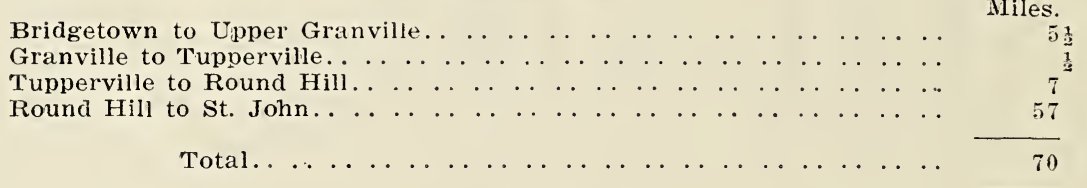

DESCRIPTION OF VESSEL EMPLOYED

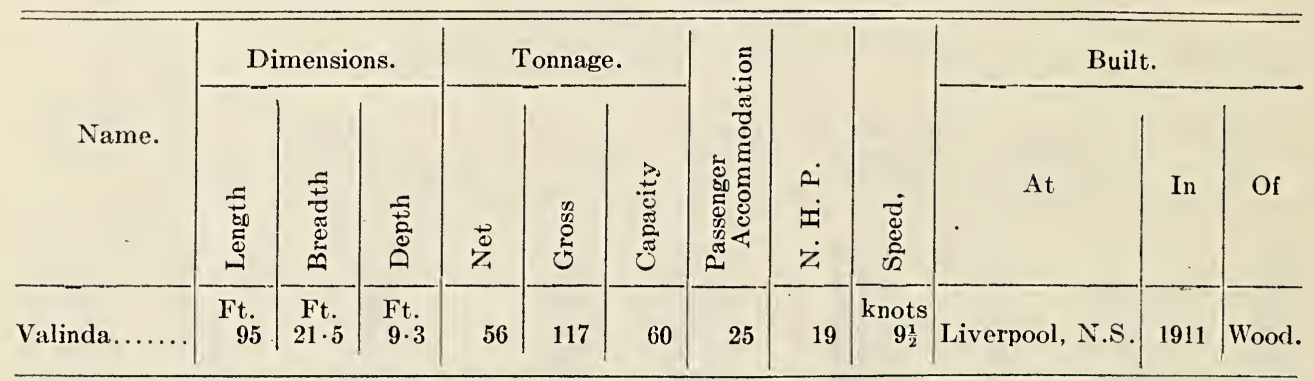

TRAFFIC RETURNS

\begin{tabular}{|c|c|c|c|c|c|c|}
\hline Calendar Year. & $\begin{array}{c}\text { No. of round } \\
\text { trips run. }\end{array}$ & $\begin{array}{l}\text { No. of } \\
\text { Passengers } \\
\text { carried. }\end{array}$ & $\begin{array}{l}\text { Tons of } \\
\text { Freight } \\
\text { carried. }\end{array}$ & $\begin{array}{l}\text { Live } \\
\text { Stock. }\end{array}$ & Mails. & $\begin{array}{l}\text { Subsidy } \\
\text { Paid. }\end{array}$ \\
\hline \multirow[t]{2}{*}{ 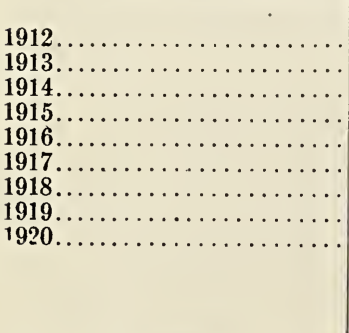 } & $\begin{array}{l}56 \\
36 \\
35 \\
36 \\
35 \\
36 \\
34 \\
37 \\
36\end{array}$ & $\begin{array}{r}7 \\
55 \\
30 \\
17 \\
29 \\
28 \\
23 \\
12 \\
8 \\
6\end{array}$ & $\begin{array}{l}5,148 \\
3,021 \\
3,845 \\
2,313 \\
2,554 \frac{1}{2} \\
2,493 \\
2,404 \\
2,964 \\
1,639 \\
1,274\end{array}$ & $\begin{array}{l}\text { Nil. } \\
\text { Nil. } \\
\text { Nil. } \\
\text { Nil. } \\
\text { Nil. } \\
\text { Nil. } \\
\text { Nil. } \\
\text { Nil. } \\
\text { Nil. } \\
\text { Nil. }\end{array}$ & $\begin{array}{l}\text { Nil. } \\
\text { Nil. } \\
\text { Nil. } \\
\text { Nil. } \\
\text { Nil. } \\
\text { Nil. } \\
\text { Nil. } \\
\text { Nil. } \\
\text { Nil. } \\
\text { Nil. }\end{array}$ & $\begin{array}{c}\$ \\
2,000 \\
2,000 \\
2,500 \\
2,500 \\
2,500 \\
2,500 \\
2,500 \\
2,500 \\
2,000\end{array}$ \\
\hline & Total....... & 14 & 2,913 & Nil. & Nil. & \\
\hline
\end{tabular}


SESSIONAL PAPER No. 10a

\section{ST. JOHN AND DIGBY}

\section{Dominion Atrantic Railway Company}

Contract No. 8 .

T. \& C. File No. 26038.

Vote 189.-St. John and Digby, steam service between-

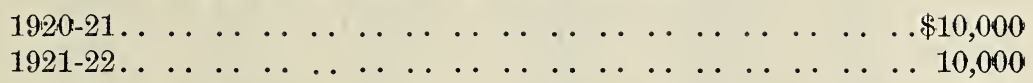

Contractors.-The Dominion Atlantic Railway Company of Kentville, N.S.

(On September 1, 1913, this line passed to the control and ownership of the Canadian Pacific Railway, Montreal, Que.)

Date of Contract.-August 25, 1920.

Duration of Contract.-April 1, 1920, to March 31, 1921.

Service and Ports of Call.-Full round daily trips (Sundays excepted) from St. John to Digby and return to St. John.

Connection at St. John.-On all trips from Digby to St. John the steamer-shall arrive at St. John in ample time for passengers, mails and perishable express goods westward bound to be transferred to the Canadian Pacific Railway afternoon train for Montreal..

In the event of any trip from Digby to St. John or the connection with the westbound trains at St. John being missed owing to stress of weather, upon satisfactory evidence thereof being furnished to the minister, he may direct that no deductions be made from the subsidy.

Government Wharves.-Steamer must call whenever possible.

Subsidy. $-\$ 10,000$, payable quarterly, in 'July, October, January and April.

Mails.-To be carried free. If during the continuance of this contract other trips are made than as above stated, all mails tendered shall be carried without additional remuneration.

Distance.-St. Johu to Digby, 47 miles. -

DESCRIPTION OF VESSEL EMPLOYED

\begin{tabular}{|c|c|c|c|c|c|c|c|c|c|c|c|c|}
\hline \multirow[b]{2}{*}{ Name. } & \multicolumn{3}{|c|}{ Dimensions. } & \multicolumn{3}{|c|}{ Tonnage. } & \multirow{2}{*}{ 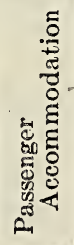 } & \multirow[b]{2}{*}{$\begin{array}{l}\dot{A} \\
\dot{Z} \\
\dot{z}\end{array}$} & \multirow[b]{2}{*}{ 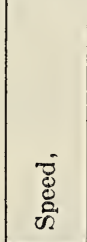 } & \multicolumn{3}{|c|}{ Built. } \\
\hline & 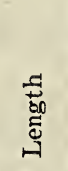 & 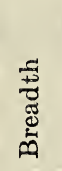 & 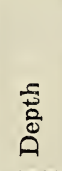 & $\begin{array}{l}\overrightarrow{0} \\
\ddot{Z}\end{array}$ & 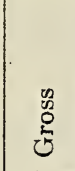 & 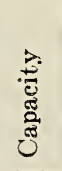 & & & & At & In & Of \\
\hline Empress. & Ft. & $\mathrm{Ft}_{34}$ & $\mathrm{Ft}_{28}$ & 612 & 1,341 & 500 & 450 & 365 & $\begin{array}{c}\text { knots } \\
16 \frac{1}{2}\end{array}$ & $\begin{array}{l}\text { Newcastle-on- } \\
\text { Tyne. }\end{array}$ & 1906 & Steel \\
\hline
\end{tabular}

$10 \mathrm{a}-5 \frac{1}{2}$ 
TRAFFIC RETURNS

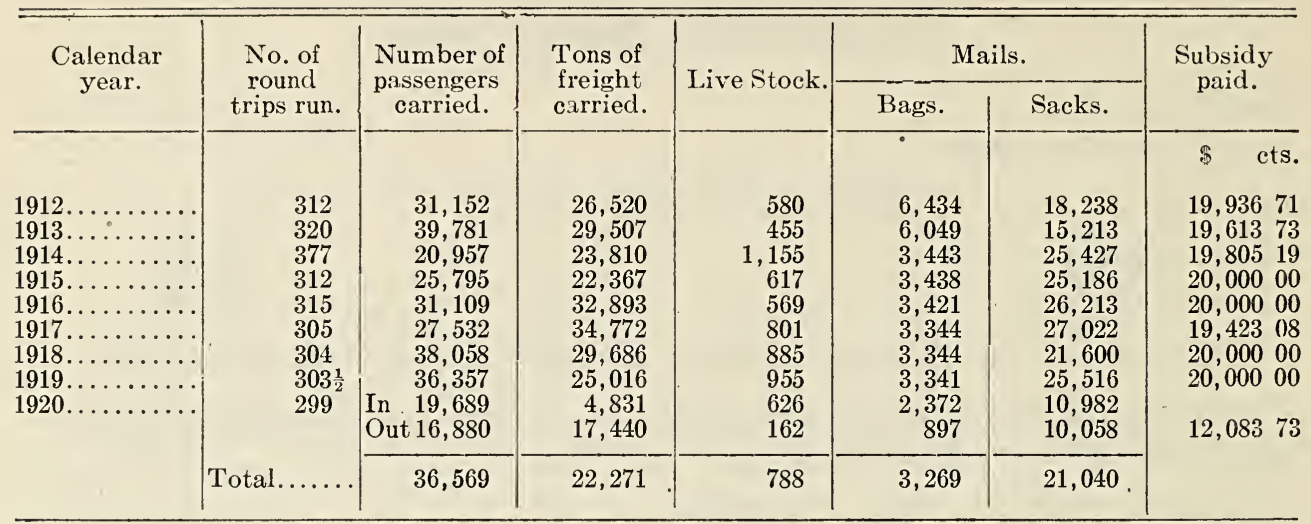

\section{ST. JOHN, DIGBY, ANAAPOLIS AND GRANVILLE}

\section{Valley Steamship Company, Ltd.}

Contract No. 37.

T. \& C. File 25486 .

Vote 190.-St. John, Digby, Annapolis and Granville, steam service between, viz.: along the west coast of the Annapolis basin-

$$
\begin{aligned}
& 1920-21 \ldots \ldots \ldots \ldots \ldots \ldots \ldots \text {. . . . . . . . . . . . . } \$ 2,000
\end{aligned}
$$

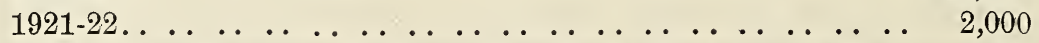

Contractors.-The Valley Steamship Co., Ltd., of Granville Ferry, N.S.

Date of Contract.-June 17, 1920.

Duration of Contract.-April 1, 1920, to March 31, 1921.

Service and Ports of Call.-A weekly service between St. John, N.B., Annapolis Royal and Granville Ferry, N.S., calling on all trips both ways at Victoria Beach and Littlewoods Wharf (opposite Goat Island), and when tide and weather will permit continuing such trips to Granville Centre, with the privilege on the part of the contractors of further extending the route to Bridgetown, N.S.

During the winter months, in case the ice will not permit the running of the vessel up the Annapolis basin and river, the trip from St. John shall be allowed to terminate at Digby.

Repairs.-The contractors have the right at any time to withdraw the steamer for a period of two weeks, and also in addition thereto for a period not exceeding 14 days, for the purpose of inspection or repairs, and may also replace it with another steamer subject to the approval of the minister.

Government Wharves.- Steamer must call whenever possible.

Mails.-To be carried free.

Subsidy. $-\$ 2,000$ per annum, payable quarterly in July, October, January and April. 
DESCRIPTION OF VESSEL EMPLOYED

\begin{tabular}{|c|c|c|c|c|c|c|c|c|c|c|c|c|}
\hline \multirow[b]{2}{*}{ Name. } & \multicolumn{3}{|c|}{ Dimensions. } & \multicolumn{3}{|c|}{ Tonnage. } & \multirow{2}{*}{ 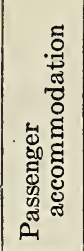 } & \multirow[b]{2}{*}{$\begin{array}{l}\dot{A} \\
\dot{\mu} \\
\dot{z}\end{array}$} & \multirow[b]{2}{*}{ 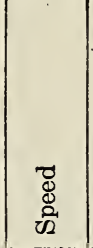 } & \multicolumn{3}{|c|}{ Built. } \\
\hline & 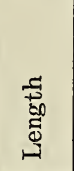 & 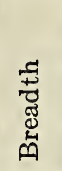 & 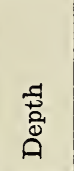 & 岂 & $\begin{array}{l}\text { on } \\
0 \\
0 \\
0 \\
0\end{array}$ & 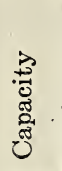 & & & & At & In & Of \\
\hline Granville..... & $\begin{array}{c}\text { Ft. } \\
112\end{array}$ & $\begin{array}{r}\text { Ft. } \\
22\end{array}$ & $\begin{array}{r}\text { Ft. } \\
9\end{array}$ & 49 & 139 & 100 & 38 & 24 & $\begin{array}{c}\text { Knots } \\
9\end{array}$ & Shelburne.. & 1909 & Wood. \\
\hline
\end{tabular}

TRAFFIC RETURNS

\begin{tabular}{|c|c|c|c|c|c|c|}
\hline Calendar year. & $\begin{array}{l}\text { No. of round } \\
\text { trips run. }\end{array}$ & $\begin{array}{l}\text { No. of } \\
\text { passengers } \\
\text { carried. }\end{array}$ & $\begin{array}{l}\text { Tons } \\
\text { freight. }\end{array}$ & $\begin{array}{l}\text { Live } \\
\text { stock. }\end{array}$ & Mail. & $\begin{array}{l}\text { Subsidy } \\
\text { paid. }\end{array}$ \\
\hline \multirow[t]{2}{*}{$\begin{array}{l}1912 . . \\
1913 . \\
1914 . \\
1915 . \\
1916 . \\
1917 . \\
1918 . \\
1919 . \\
1920 .\end{array}$} & \multirow[t]{2}{*}{$\begin{array}{l}50 \\
51 \\
52 \\
50 \\
50 \\
45 \\
44 \\
52 \\
52\end{array}$} & $\begin{array}{cc} & 66 \\
& 57 \\
& 69 \\
& 39 \\
& 37 \\
& 78 \\
& 78 \\
& 74 \\
& 51 \\
\text { In } & 55 \\
\text { Out } & 40\end{array}$ & $\begin{array}{r}3,386 \\
2,788 \\
3,024 \\
3,079 \\
4,069 \\
3,299 \\
3,976 \\
3,807 \\
2,415 \\
782\end{array}$ & $\begin{array}{l}\text { Nil.. } \\
\text { Nil. } \\
\text { Nil. } \\
\text { Nil. } \\
\text { Nil. } \\
\text { Nil. } \\
\text { Nil. } \\
\text { Nil. } \\
\text { Nil. } \\
\text { Nil. }\end{array}$ & $\begin{array}{l}\text { Nil. } \\
\text { Nil. } \\
\text { Nil. } \\
\text { Nil. } \\
\text { Nil. } \\
\text { Nil. } \\
\text { Nil. } \\
\text { Nil. } \\
\text { Nil. } \\
\text { Nil. }\end{array}$ & $\begin{array}{rr}\$ & \text { cts. } \\
1,500 & 00 \\
1,500 & 00 \\
1,875 & 00 \\
1,961 & 55 \\
2,000 & 00 \\
1,875 & 00 \\
2,000 & 00 \\
2,000 & 00 \\
2,000 & 00\end{array}$ \\
\hline & & 95 & 3,197 & Nil. & Nil. & - \\
\hline
\end{tabular}

ST. JOHN AND PORTS ON THE BAY OF FUNDY AND MINAS BASIN

St. John Steamship Co., Ltd.

Contract No. 71.A.

T. \& C. File 25520.

Vote 191.-St. John, N.B., and ports on the Bay of Fundy and Minas Basin, steam service between-

$$
\begin{aligned}
& 1920-21 \ldots \ldots \ldots \ldots \ldots \ldots \text {. . . . . . . . . . . . . . } \$ 5,000 \\
& 1921-22 \ldots \ldots \ldots \ldots \ldots \ldots \ldots \ldots \ldots \ldots \ldots
\end{aligned}
$$

Contractors.-The St. John Steamship Co., Ltd., of St. John, N.B.

Date of Contract.-April 20, 1920.

Druration of Contract.-From the opening to the closing of navigation, 1920 . Service and Ports of Call-

(a) A regular service every two weeks between St. John, N.B., and Windsor, N.S., calling each way at Canning, Wolfville, Cheverie and Noel.

(b) A regular service every two weeks, alternating with the service specified in section (a) aforesaid, between St. John, N.B., and Maitland, N.S., calling at Spencer's Island, Parrsboro', Port Greville and Bass River.

(c) The minimum number of trips to be made during the season of navigation shall be 28 . 
Subsidy. $-\$ 5,000$ per annum, payable in equal instalments on August 1 , October 1 , and at the close of navigation.

Mails.-To be carried free.

DISTANTES

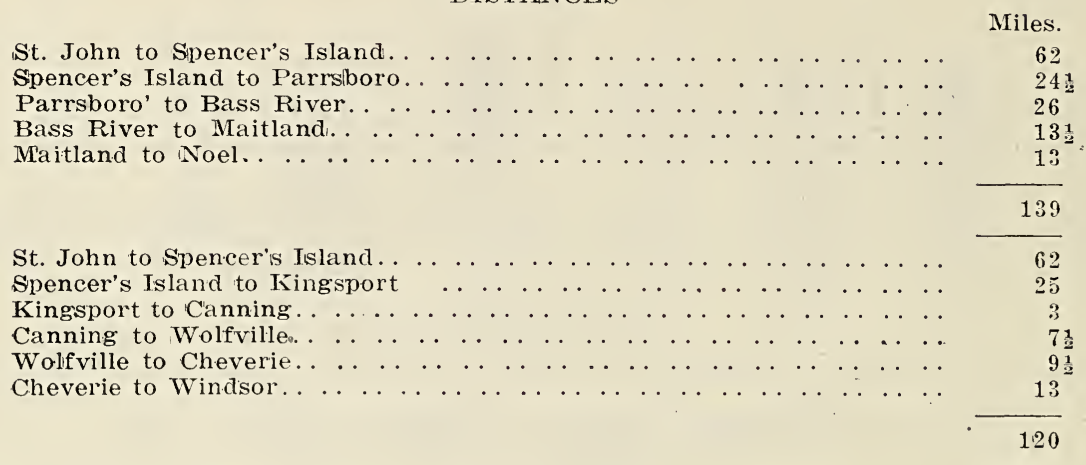

DESCRIPTION OF VESSEL EMPLOYED

\begin{tabular}{|c|c|c|c|c|c|c|c|c|c|c|c|c|}
\hline \multirow[b]{2}{*}{ Name. } & \multicolumn{3}{|c|}{ Dimensions. } & \multicolumn{3}{|c|}{ Tonnage. } & \multirow{2}{*}{ 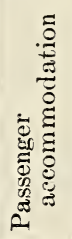 } & \multirow[b]{2}{*}{$\begin{array}{l}\dot{2} \\
\dot{I} \\
\dot{z}\end{array}$} & \multirow[b]{2}{*}{ 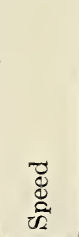 } & \multicolumn{3}{|c|}{ Built. } \\
\hline & 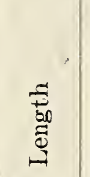 & 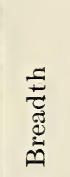 & 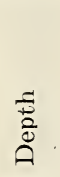 & ¿ & $\begin{array}{l}n \\
0 \\
0 \\
0 \\
0\end{array}$ & 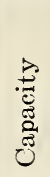 & & & & At & In & Of \\
\hline Glenholme. & $\begin{array}{c}\text { Ft. } \\
102 \cdot 5\end{array}$ & $\begin{array}{l}\mathrm{Ft} . \\
30.5\end{array}$ & $\begin{array}{l}\text { Ft. } \\
9 \cdot 7\end{array}$ & 125 & 233 & & 7 & 24 & $\begin{array}{c}\text { Knots } \\
8 \frac{1}{2}\end{array}$ & Yarmouth, N.S. & 1919 & Wood \\
\hline
\end{tabular}

TRAFFIC RETURNS

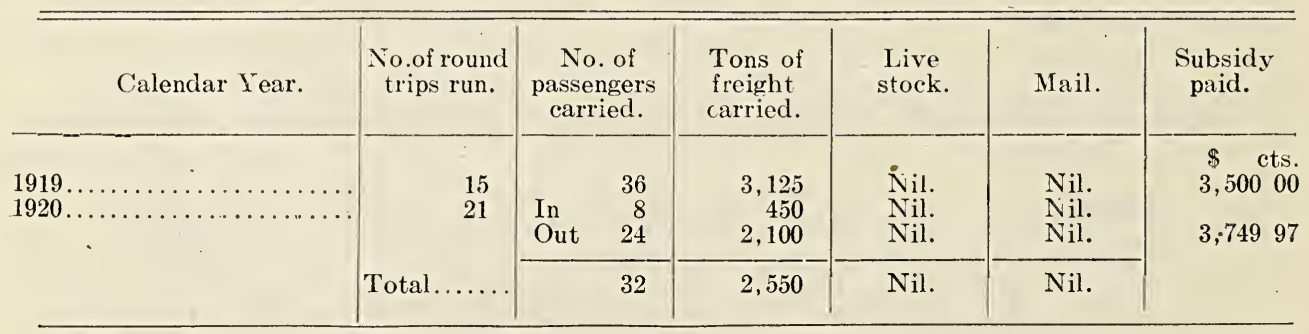

ST. JOHN, WESTPORT AND YARMOUTH AND OTHER WAY PORTS

Hugh Cany \& Sox, Ltid.

Contract No. $42 \mathrm{~A}$.

T. \& C. File No. 25533.

Vote 192.-St. John, Westport and Yarmouth and other way ports, steam service between-

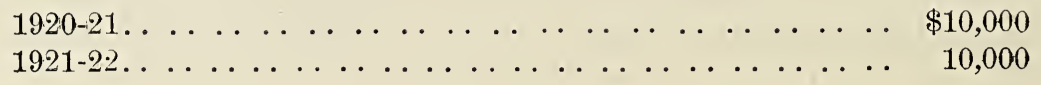


SESSIONAL PAPER NO. 10a

Contractors.-Hugh Cann \& Son, Ltd., of Yarmouth, N.S.

Date of Contract.-April 14, 1920.

Duration of Contract.-April 1, 1920, to March 31, 1921.

Service and Ports of Call.- Seventy round trips are to be made during the period covered by the contract, between St. John, Westport and Yarmouth, calling on each trip both ways at Freeport and Tiverton, unless ice prevents.

Four round trips are to be made in each of the months of April, November, December, January, February and March; 6 round trips in the month of May; and 8 round trips in each of the months of June, July, August, September and October.

Government Wharves.-Steamer must call whenever possible.

Subsidy. $-\$ 10,000$, payable as follows: $\$ 142.86$ for each round trip performed, to be paid at the close of June, September, December and March.

Mails.-To be carried free.

DISTANCES.

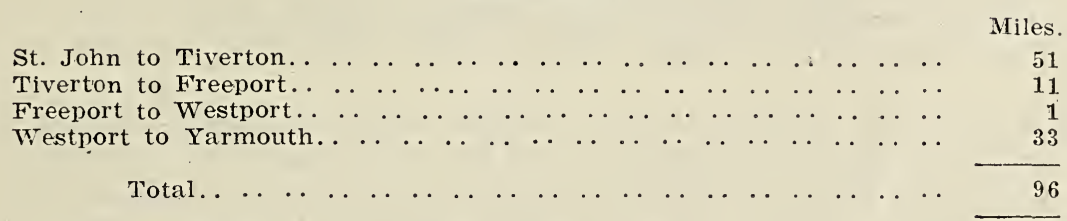

DESCRIPTION OF VESSEL EMPLOYED.

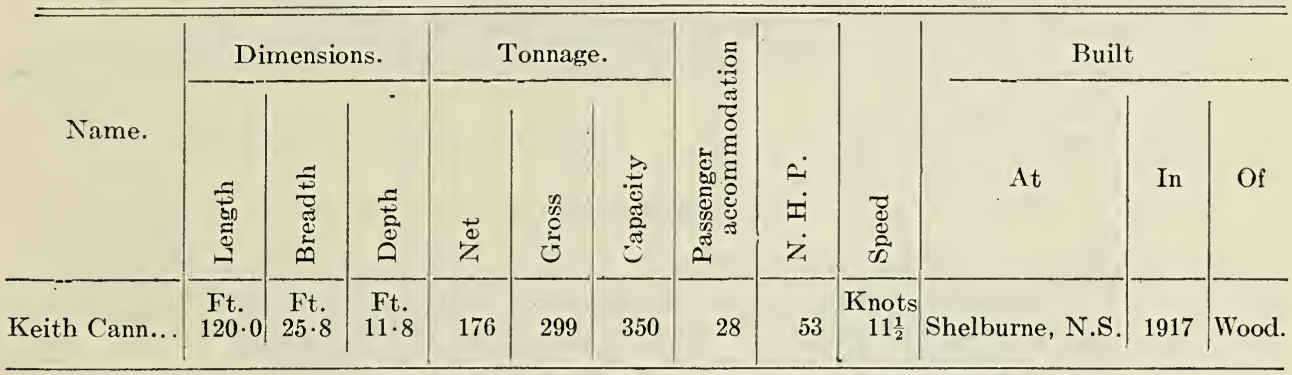

TRAFFIC RETURNS.

\begin{tabular}{|c|c|c|c|c|c|c|}
\hline Calendar year. & $\begin{array}{l}\text { No. of round } \\
\text { trips run. }\end{array}$ & $\begin{array}{l}\text { No. of } \\
\text { passengers } \\
\text { carried. }\end{array}$ & $\begin{array}{l}\text { Tons of } \\
\text { freight } \\
\text { carried. }\end{array}$ & $\begin{array}{l}\text { Live } \\
\text { stock. }\end{array}$ & $\begin{array}{l}\text { Bags } \\
\text { mail. }\end{array}$ & $\begin{array}{l}\text { Subsidy } \\
\text { paid. }\end{array}$ \\
\hline \multirow[t]{2}{*}{$\begin{array}{l}1913 . . \\
1914 . . \\
1915 . . \\
1916 . . \\
1917 . . \\
1918 . . \\
1919 . . \\
1920 . .\end{array}$} & \multirow[t]{2}{*}{$\begin{array}{l}36 \frac{1}{2} \\
81 \\
79 \frac{1}{2} \\
79 \frac{1}{2} \\
78 \frac{1}{2} \\
75 \frac{1}{2} \\
78 . \\
76 \frac{1}{2}\end{array}$} & $\begin{array}{r}408 \\
959 \\
\\
1,192 \\
1,258 \\
1,290 \\
1,293 \\
\quad 2,116 \\
\text { In } 1,127 \\
\text { Out } 1,023\end{array}$ & $\begin{array}{r}1,808 \\
4,497 \\
5,684 \\
5,723 \\
6,598 \\
11,820 \\
11,096 \\
2,447 \\
6,996\end{array}$ & $\begin{array}{l}\text { Nil. } \\
\text { Nil. } \\
\text { Nil. } \\
\text { Nil. } \\
\text { Nil. } \\
\text { Nil. } \\
\text { Nil. } \\
\text { Nil. } \\
\text { Nil. }\end{array}$ & $\begin{array}{l}\text { Nil. } \\
\text { Nil. } \\
\text { Niil. } \\
\text { Nil. } \\
\text { Nil. } \\
\text { Nil. } \\
\text { Nil. } \\
\text { Nil. } \\
\text { Nil. }\end{array}$ & \multirow[t]{2}{*}{ 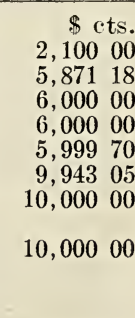 } \\
\hline & & 2,150 & 9,443 & Nil. & Nil. & \\
\hline
\end{tabular}




\section{SYDNEY AND BAY ST. LAWRENCE}

The North Shore Steamship Company, Ltd.

Contract No. 33.

T. \& C. File No. 25540.

Vote 193.-Sydney and Bay St. Lawrence, calling at way ports, steam service between-

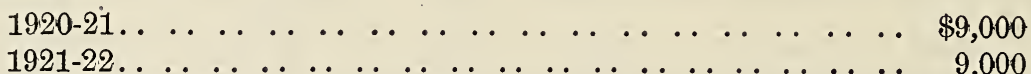

Contractors.-The North Shore Steamship Company, Limited, of Sydney, N.S.

Date of Contract.-April 21, 1920.

Duration of Contract.-From the opening to the close of navigation in the year 1920.

Services and Ports of Call.-From the opening of navigation until June 15, and from October 15 until the close of navigation, one full round trip each week between Sydney and St. Anne's Bay, calling both going and returning at North Sydney, Breton Cove, Englishtown and North River; and one full round trip each week between Sydney and Bay St. Lawrence, calling at North Sydney, Ingonish, Neil's Harbour and Dingwall; returning calling at Neil's Harbour, Ingonish and North Sydney.

From June 15 to October 15 two full trips each week between Sydney and Ste. Anne's Bay, with calls as above given; and two full round trips each week between Sydney and Neil's Harbour, calling both going and returning at North Sydney and Ingonish, one trip each week to be extended to Bay St. Lawrence, with calls on the outward trip at Dingwall and Cape North. The Friday trip to Ingonish and Neil's Harbour is to be extended to Dingwall during July and August.

Government Wharves.-Steamer must call whenever possible.

Subsidy. $\$ 9,000$, payable in instalments in June, August and October, and on the close of navigation.

Mails.-To be carried free.

DISTANCES.

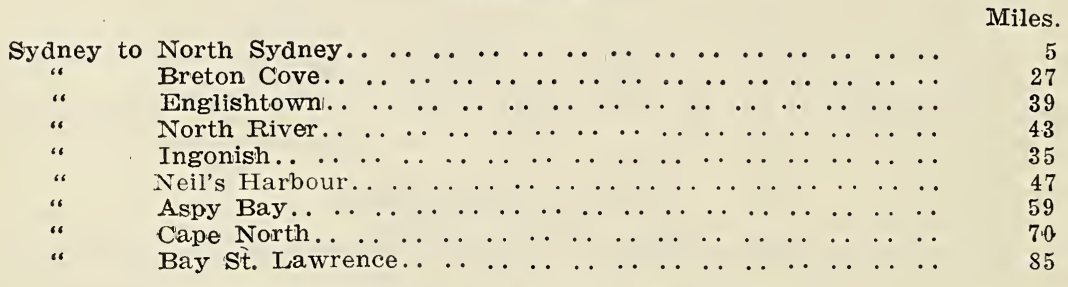

DESCRIPTION OF VESSEL EMPLOYED.

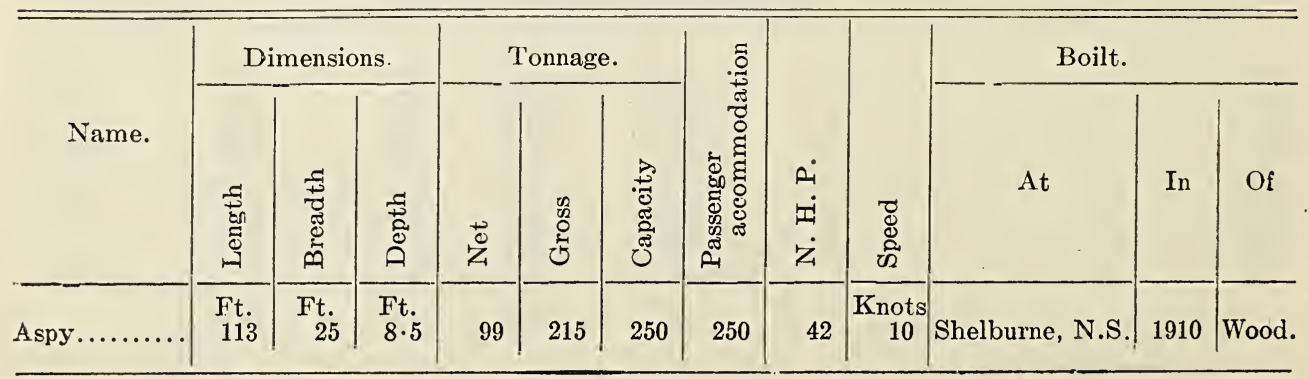


TRAFFIC RETURNS.

\begin{tabular}{|c|c|c|c|c|c|c|}
\hline Calendar year. & $\begin{array}{c}\text { No. of round } \\
\text { trips run. }\end{array}$ & $\begin{array}{c}\text { No. of } \\
\text { passengers } \\
\text { carried. }\end{array}$ & $\begin{array}{l}\text { Tons of } \\
\text { freight } \\
\text { carried. }\end{array}$ & $\begin{array}{l}\text { Live } \\
\text { stock. }\end{array}$ & Mails. & $\begin{array}{l}\text { Subsidy } \\
\text { Paid. }\end{array}$ \\
\hline \multirow[t]{2}{*}{ 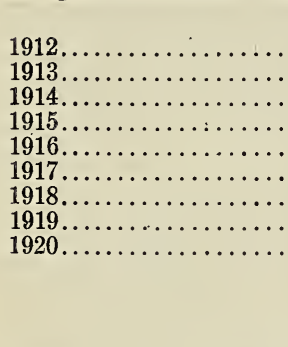 } & $\begin{array}{l}112 \\
122 \\
110 \\
112 \\
110 \\
114 \\
113 \\
112 \\
116\end{array}$ & $\begin{array}{r}2,550 \\
2,300 \\
1,715 \\
1,360 \\
1,510 \\
1,515 \\
1,680 \\
\quad 1,860 \\
\text { In } 725 \\
\text { Out } 1,175\end{array}$ & $\begin{array}{r}890 \\
960 \\
860 \\
765 \\
835 \\
1,120 \\
1,260 \\
1,640 \\
575 \\
1,360\end{array}$ & $\begin{array}{r}160 \\
95 \\
45 \\
130 \\
45 \\
55 \\
226 \\
70 \\
90 \\
\text { Nil. }\end{array}$ & $\begin{array}{l}\text { Nil. } \\
\text { Nil. } \\
\text { Nil. } \\
\text { Nil. } \\
\text { Nil. } \\
\text { Nil. } \\
\text { Nil. } \\
\text { Nil. } \\
\text { Nil. } \\
\text { Nil. }\end{array}$ & $\begin{array}{l}\$ \\
6,500 \\
6,000 \\
6,000 \\
6,000 \\
6,000 \\
6,000 \\
6,000 \\
6,000 \\
9,000\end{array}$ \\
\hline & $\begin{array}{c}116 \\
\text { Total....... }\end{array}$ & 1,900 & 1,935 & 90 & Nil. & \\
\hline
\end{tabular}

\section{SYDNEY AND WHYCOCOMAGH}

The Bras d'Or Steamboat Company, Ltd.

Contract No. 34 .

T. \& C. File No. 25544.

Vote 194.-Sydney and Whycocomagh, steam service between-

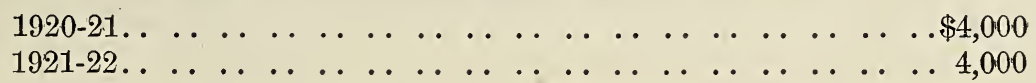
N.S.

Contractors.-The Bras d'Or Steamboat Company, Limited, of North Sydney,

Date of Contract.-April 22, 1920.

Duration of Contract.-From the opening to the close of navigation in 1920.

Service and Ports of Call.-From the opening of navigation to June 30, and from October 18 to close of navigation, two full round trips each week; and from June 30 to September 30, three full round trips each week, between Sydney and Whycocomagh, calling both going and returning at North Sydney, Big Bras d'Or, New Campbellton, Boularderie, Ross Ferry, Big Harbour, Kempt Head, Baddeck, Washabuck Centre, Nyanza and Little Narrows.

It is agreed that if, upon due and proper investigation, the Minister should deem it advisable that three round trips a week should be performed after September 30, the contractors will perform the said three round trips each week, until otherwise instructed by the Minister.

It is further agreed that from October 15 to the close of navigation one call each week shall be made at Iona.

Subsidy. $-\$ 4,000$, payable at the rate of $\$ 50$ per trip for each round trip performed up to maximum of 80 round trips.

Government Wharves.-Steamer must call whenever possible.

Mails.-To be carried free. 
11 GEORGE V, A. 1921

DISTANCES.

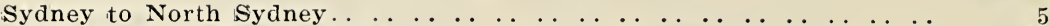

North Sydney to Big Bras d'Or.. $\ldots \ldots \ldots \ldots$

Big Bras d'Or to New Campbellton. . . . . . . . . . . . . . . . . . . . .

New Campbellton to Boularderie Centre. . . . . . . . . . . . . . . . . . .

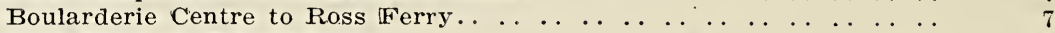

Ross Ferry to Big Harbour.. . . . . . . . . . . . . . . . . . . . . . . .

Big Harbour to Kempt Head. . . . . . . . . . . . . . . . . . . . . . . . . . . .

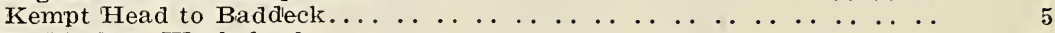

Baddeck to Washabuck. . . . . . . . . . . . . . . . . . . . . . . . . . 5

Washabuck to Nyanza.. . . . . . . . . . . . . . . . . . . . . . . . .

Nyanza to Little Narrows.

Little Narrows to Whycocomagh. . . $\ldots \ldots \ldots \ldots \ldots$

Total. . . . . . . . . . . . . . . . . . . . . .

DESCRIPTION OF VESSEL EMPLOYED.

\begin{tabular}{|c|c|c|c|c|c|c|c|c|c|c|c|c|}
\hline \multirow[b]{2}{*}{ Name. } & \multicolumn{3}{|c|}{ Dimensions. } & \multicolumn{3}{|c|}{ Tonnage. } & \multirow{2}{*}{ 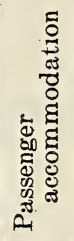 } & \multirow[b]{2}{*}{$\begin{array}{l}\dot{A} \\
\dot{I} \\
\dot{z}\end{array}$} & \multirow[b]{2}{*}{ 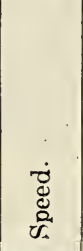 } & \multicolumn{3}{|c|}{ Built. } \\
\hline & 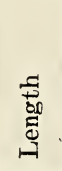 & 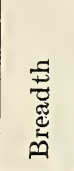 & $\begin{array}{l}\text { 芩 } \\
\text { هัँ }\end{array}$ & 莒 & 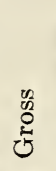 & 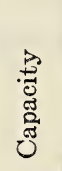 & & & & At & In & Of \\
\hline Marion. & $\begin{array}{l}\text { Ft. } \\
150\end{array}$ & $\begin{array}{l}\text { Ft. } \\
26 \cdot 5\end{array}$ & $\begin{array}{r}\text { Ft. } \\
8\end{array}$ & 269 & 478 & 100 & 400 & 49 & $\begin{array}{c}\text { Knots } \\
12\end{array}$ & $\begin{array}{c}\text { New York, } \\
\text { U.S.A. }\end{array}$ & 1876 & Wood. \\
\hline
\end{tabular}

TRAFFIC RETURNS.

\begin{tabular}{|c|c|c|c|c|c|c|}
\hline Calendar year. & $\begin{array}{l}\text { No.of round } \\
\text { trips run. }\end{array}$ & $\begin{array}{l}\text { No. of } \\
\text { passengers } \\
\text { carried. }\end{array}$ & $\begin{array}{l}\text { Tons of } \\
\text { freight } \\
\text { carried. }\end{array}$ & $\begin{array}{l}\text { Live } \\
\text { stock. }\end{array}$ & Mails. & $\begin{array}{l}\text { Subsidy } \\
\text { paid. }\end{array}$ \\
\hline \multirow[t]{2}{*}{$\begin{array}{l}1912 . \\
1913 . \\
1914 . \\
1915 . \\
1916 . \\
1917 . \\
1918 . \\
1919 . \\
1920 .\end{array}$} & $\begin{array}{l}84 \\
89 \\
78 \\
80 \\
81 \\
79 \\
79 \\
81 \\
82\end{array}$ & $\begin{array}{r}5,919 \\
6,404 \\
5,879 \\
5,773 \\
6,655 \\
6,399 \\
4,756 \\
6,976 \\
\text { In } 2,403 \\
\text { Out } 2,670\end{array}$ & $\begin{array}{l}1,820 \\
1,607 \\
1,554 \\
1,556 \\
1,794 \\
2,029 \\
2,319 \\
4,042 \\
1,779 \\
1,763\end{array}$ & $\begin{array}{r}4,693 \\
5,343 \\
5,458 \\
4,081 \\
3,631 \\
2,674 \\
2,848 \\
2,621 \\
2,381 \\
187\end{array}$ & $\begin{array}{l}\text { Nil. } \\
\text { Nil. } \\
\text { Nil. } \\
\text { Nil. } \\
\text { Nil. } \\
\text { Nil. } \\
\text { Nil. } \\
\text { Nil. } \\
\text { Nil. } \\
\text { Nil. }\end{array}$ & \begin{tabular}{lc}
\multicolumn{3}{c}{$\$$} \\
3,000 & 00 \\
3,000 & 00 \\
3,000 & 00 \\
3,000 & 00 \\
3,000 & 00 \\
3,000 & 00 \\
3,000 & 00 \\
4,000 & 00 \\
4,000 & 00
\end{tabular} \\
\hline & Total. & 5,073 & 2,542 & 2,568 & Nil. & \\
\hline
\end{tabular}

SYDNEY, BRAS D'OR LAKES, AND CAPE BRETON PORTS

The Coastal Steamship Conpany, Ltrd.

Contract No. 77 .

T. \& C. File 25539.

Vote 195.-Sydney and Bras d'Or Lake Ports, and Ports on the East and West Coasts of Cape Breton, steam service between-

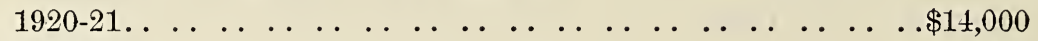

$1921-22 \ldots \ldots \ldots \ldots \ldots \ldots \ldots 14,000$ 
SESSIONAL PAPER No. 10a

Contractors.-The Coastal Steamship Company, Ltd., of Sydney, N.S.

Date of Contract.-April 24, 1920.

Duration of Contract.-From the opening to the close of navigation, 1920.

Service and Ports of Call.-Weekly, from Sydney to North Sydney, Baddeck, Grand Narrows, East Bay, Big Pond, Irish Cove, Johnston's Harbour, Marble Mountain, St. Peter's, Grandique, Poulamond, L'Ardoise, Arichat, Mulgrave, Hawkesbury, Hastings, Port Hood, Margaree, Grand Etang and Cheticamp, returning to Sydney and proceeding thence to Gabarous, calling at Port Morien, Main-à-dieu and Louisburg.

(a) If it be found impossible for the Bras d'Or to make weekly calls at Port Morien, Main-à-dieu, Louisburg and Gabarous, a reduced service to the said ports may be performed. The contractors, however, shall use their best endeavours to perform a weekly service to the said ports, if time permits.

Subsidy. - $\$ 14,000$ per annum, payable in instalments on July 1, October 1, and at the close of navigation.

Mails.-To be carried free.

DISTANCES.

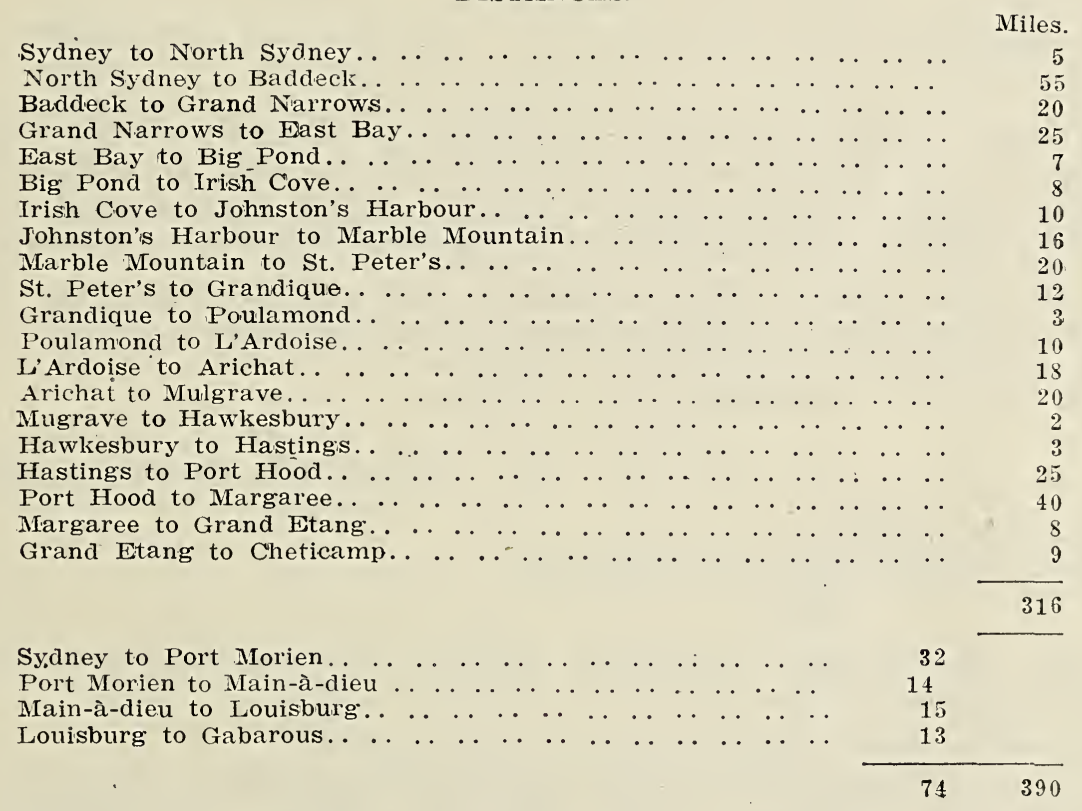

DESCRIPTION OF VESSEL EMPLOYED.

\begin{tabular}{|c|c|c|c|c|c|c|c|c|c|c|c|c|}
\hline \multirow[b]{2}{*}{ Name. } & \multicolumn{3}{|c|}{ Dimensions. } & \multicolumn{3}{|c|}{ Tonnage. } & \multirow{2}{*}{ 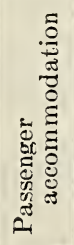 } & \multirow[b]{2}{*}{$\begin{array}{l}\dot{A} \\
\dot{Z} \\
\dot{Z}\end{array}$} & \multirow[b]{2}{*}{ 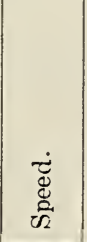 } & \multicolumn{3}{|c|}{ Built. } \\
\hline & 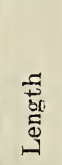 & 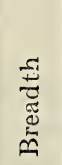 & $\begin{array}{l}\text { 苋 } \\
\stackrel{0}{0}\end{array}$ & $\begin{array}{l}\ddot{0} \\
z\end{array}$ & 常 & 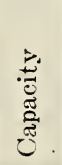 & & & & At & In & Of \\
\hline Bras d'Or.. & $\begin{array}{l}\text { Ft. } \\
128\end{array}$ & $\begin{array}{r}\mathrm{Ft} . \\
28\end{array}$ & Ft. & 136 & 371 & 400 & 250 & 71 & $\begin{array}{c}\text { Knots } \\
13\end{array}$ & $\begin{array}{l}\text { Mahone Bay, } \\
\text { N.S. }\end{array}$ & 1919 & Wood. \\
\hline
\end{tabular}


TRAFFIC RETURNS.

\begin{tabular}{|c|c|c|c|c|c|c|c|c|}
\hline \multirow{2}{*}{ Calendar year. } & \multirow{2}{*}{$\begin{array}{l}\text { No. of } \\
\text { round } \\
\text { trips run. }\end{array}$} & \multirow{2}{*}{$\begin{array}{l}\text { No. of } \\
\text { passengers } \\
\text { carried. }\end{array}$} & \multicolumn{2}{|c|}{$\begin{array}{l}\text { Tons of freight } \\
\text { Carried. }\end{array}$} & \multirow{2}{*}{$\begin{array}{l}\text { Live } \\
\text { stock. }\end{array}$} & \multicolumn{2}{|c|}{ Mails. } & \multirow{2}{*}{$\begin{array}{l}\text { Subsidy } \\
\text { paid. }\end{array}$} \\
\hline & & & Weight. & $\begin{array}{l}\text { Measure- } \\
\text { ment. }\end{array}$ & & $\begin{array}{l}\text { Lock } \\
\text { bags. }\end{array}$ & $\begin{array}{l}\text { Tied } \\
\text { sacks. }\end{array}$ & \\
\hline \multirow[t]{2}{*}{$\begin{array}{l}1919 . . \\
1920 . .\end{array}$} & \multirow[t]{2}{*}{$\begin{array}{l}36 \\
35\end{array}$} & $\begin{array}{lr} & 45 \\
\text { In } & 560 \\
\text { Out } & 500\end{array}$ & $\begin{array}{r}1,825 \\
725 \\
2,675\end{array}$ & $\begin{array}{l}\text { Nil. } \\
\text { Nil. } \\
\text { Nil. }\end{array}$ & $\begin{array}{l}\text { Nil. } \\
\text { Nil. } \\
\text { Nil. }\end{array}$ & $\begin{array}{l}\text { Nil. } \\
\text { Nil. } \\
\text { Nil. }\end{array}$ & $\begin{array}{l}\text { Nil. } \\
\text { Nil. } \\
\text { Nil. }\end{array}$ & \multirow{2}{*}{$\begin{array}{r}8,42064 \\
14,00000\end{array}$} \\
\hline & & 1,060 & 3,400 & Nil. & Nil. & Nil. & Nil. & \\
\hline
\end{tabular}

\section{SUPERVISION OF SUBSIDIZED STEAMSHIP SERVICES}

Vote 196.-Expenses in connection with the supervision of Subsidized Steamship Services-

$$
\begin{array}{lllllllllllllllllll}
1920-21 \ldots & \ldots & \ldots & \ldots & \ldots & \ldots & \ldots & \ldots & \ldots & \ldots & \ldots & \ldots & \ldots & \ldots & \ldots & \ldots & \ldots & \$ 4,000 \\
1921-22 & \ldots & \ldots & \ldots & \ldots & \ldots & \ldots & \ldots & \ldots & \ldots & \ldots & \ldots & \ldots & \ldots & \ldots & \ldots & \ldots & \ldots & 4,000
\end{array}
$$

By Order in Council of April 10, 1912, Mr. W. E. Tupper, of Digby, N.S., was appointed Supervising Officer of Subsidized Steamship Services. His salary is $\$ 2,640$ per annum, and he is allowed the usual travelling and other contingent expenses. The title of this position has been changed to "Inspector of Subsidized Steamships."

In December, 1916, Mr. Tupper enlisted for active service overseas. He returned to Canada in 1918, and resumed his position as supervising officer on January 1, 1919.

The Inspector's Annual Report is as follows:-

DigBY, N.S., February 5, 1921.

F. C. T. O'Hara, Esq.,

Deputy Minister, Trade and Commerce,

Ottawa.

Dear Sir,-I beg to submit my report on Subsidized Steamship Services for the year ending December 31, 1920.

During the season twenty-six services were inspected.

The services were, with very few exceptions, well performed. Captains carried out their duties faithfully and conscientiously; passenger accommodations and freight capacity of steamers in practically every case were found adequate to requirements, and, taking into consideration the nature of several of the services, the food was of good quality and the meals well served.

Compared with the year 1919, it is considered that a marked improvement was effected in the quality of several of the services. This was noticeably the case in respect to the following:-

Charlottetown, Victoria and Holliday's Wharf.-In 1918 the ss. Harland, built especially for the route, was sold to the contractors for the Pictou, Montague, Murray: Harbour and Georgetown service; in consequence of which the department was compelled to sanction the employment of a less adaptable boat. In 1920, however, the original owners repurchased the Harland, and she is again performing the service for which she was originally built. 
Pictou, Montague, Murray Harbour and Georgetown.-During 1918 and 1919 this service was performed by the Harland. Although possessing adequate freight capacity and excellent passenger accommodation, this steamer, built for river service only, was unadapted to "outside" work. Last year the contract was awarded the Georgetown Steamship Company, Pictou, N.S., which placed the ss. Magdalen on the route. After being acquired by the contractors this steamer underwent extensive alterations and repairs, and is giving excellent satisfaction.

Petit de Grat and I.C.R. terminus at Mulgrave.-At the close of the season of 1919, Mr. William Leslie, who had operated this service for a number of years, finding that there was insufficient passenger and freight traffic on the route to warrant a continuance of the service with so large a boat, failed to renew the contract. The LaHave Steamship Company, L.td., West LaHave, N.S., having tendered for and received the contract, placed the ss. Tussle on the route. The contractors were required to effect considerable improvements in respect to the passenger accommodations before the steamer was accepted by the department. The Tussle has since proved a very suitable boat.

Mulgrave and Guysboro.-In 1919, owing to the extreme scarcity of suitable tonnage (a situation which is proving of considerable embarrassment to the department), a contract was awarded for the performance of this service to the ss. Elaine. This steamer later proved inadequate. In 1920, however, the contractors procured the ss. Westport 111. The passenger accommodations on this boat were completely remodelled, and she has since been furnishing a most satisfactory service.

Sydney and the East and West Coasts of Cape Breton.-Previous to the year 1920 this service had been partially performed, for many years, by the ss. Weymouth, a steamer not only very old and unseaworthy, but absolutely inadequate to meet the demands of the route. Last year the contract was awarded the Coastal Steamship Company, Ltd., Sydney, C.B., which placed the ss. Bras d'Or on the route. This steamer was built for the service, and has not only ample carrying capacity, but by reason of the commodiousness and excellence of her passenger accommodations, may be considered superior to any boat of her class subsidized by the department for service in the coastal waters of the Maritime Provinces.

Magdalen Islands and Mainland.-This service has been unsatisfactorily performed during the last two vears. Further, it may be stated that in view of the fact that the reasons responsible for the unfortunate conditions have been so thoroughly discussed, further allusion to the subject in this report is considered unnecessary. It may be expedient to say, however, that it is my firm opinion that the satisfactory performance of this service depends primarily upon, first: the procuring of a steamer, the general dimensions and construction of which shall follow closely those of the ss. Lady Sybil, the boat which so admirably performed this service from 1909 to 1915 , inclusive; second, that the Magdalen Island merchants shall use their utmost endeavour to order their winter supplies at the earliest possible date, consistent with the somewhat arbitrary nature of their business, thereby allowing a margin of time for shipments to be transported to islands before the closing of navigation.

"The trade depression of last year has been felt very keenly by the great majority of steamship companies operating subsidized coastal services. In view of the fact that merchants doing business at outports were purchasing only necessary lines in staples, freights were naturally very light. As, however, we are looking forward to greater stability in prices in the near future, freight receipts should show a healthy increase during the coming season.

I have the honour to be, sir, Your obedient servant,

(Sgd.) W. E.TUPPER, Inspector Subsidized Steamships. 


\section{SOME CLAUSES COMMON TO ALL CONTRACTS}

Note.-Some of the principal sections common to all contracts, and as such hereinbefore frequently referred to, read as follows:-

\section{Proof of Performance of Service to be Furnished.}

The contractors shall furnish and establish at their own expense the necessary agents required for the efficient performance of this contract, and shall with diligence as soon after the completion of each voyage as may be, furnish to the minister full and complete copies of the manifests of the cargoes and list of passengers carried on each royage, duly certified by the proper officers of customs, and also such other documents, information and evidence as may be reasonably required by the minister to show the volume, extent and value of the trade carried on by the said steamers and the full performance on their part of services, requirements and conditions of this contract, in order to enable him to judge as to whether the terms of this contract have been or are being fully and faithfully carried out and complied with, within the true intent and meaning thereof, and his decision in that respect shall be binding, final and conclusive; and the furnishing of such certificates, documents and evidence as hereinbefore specified shall be a condition, precedent to the payment of the subsidy herein provided for, or any portion thereof, and if in the opinion of the minister, all the terms of this contract have not been fully complied with by the contractors, he may deduct from the subsidy otherwise payable such portion thereof as he may deem fit and proper, taking into consideration all the circumstances connected therewith, and the contractors shall at all times during the continuance of this contract well and faitlifully abide by and conform to all such requirements as may be made by the minister with regard to the said steamers in the performance of this contract.

\section{Financial Statements}

It is further understood and agreed that the contractors, whenever so required, shall furnish the minister with such financial statement or statements as he may desire from time to time respecting all revenues derived from and all expenditures in connection with the conduct of the service herein provided for.

\section{British Subjects}

It is further understood and agreed by the contractors, that two-thirds of the total number of officers, engineers, stewards, crew or other employees whatsoever upon the steamships engaged in the performance of the service herein contracted for, shall be British subjects, but the non-observance of this clause shall not constitute a violation of this contract in such individual cases as may from time to time be approved by the minister in writing.

\section{Equipment of Steamers}

The steamers to be employed as herein specified, shall at all times during the continuance of this contract be fully seaworthy, well-officered, manned, victualled, equipped, provided and furnished, having regard to the service which the contractors have hereby undertaken to perform; and shall have ample and suitable accommodation for the passengers, mails and freight to be carried over the route specified; and shall at all times carry boats and life-saving appliances in compliance with the law, and shall be in all respects to the approval of the minister. 


\section{Carriage of Mails}

The contractors shall during the performance of this contract, convey on each and every trip of the steamers performing the aforementioned services, both on outward and homeward voyages, all such mails as shall be tendered to the proper officers or persons in that behalf on the said steamers by or on behalf or under the direction of the postal authorities of Canada, or those at the terminal port or ports of call herein referred to, and shall deliver all such mails at their proper destination at the terminal port or ports of call above referred to; and the expenses of carrying such mails from the post offices or railway stations to the steamers and from the steamers to the post offices or railway stations at the terminal ports and at the ports of call shall be borne by the contractors, who will be subject to all general and special regulations now or hereafter existing during the continuance of this contract in connection with the postal service. For the conveyance of all such mails no payment shall be made or required over or beyond the amount of subsidy herein mentioned or provided for.

\section{Accommodation for Mails}

The said steamer shall be provided with sufficient and convenient accommodation and protection for all such mails, to the satisfaction of the Honourable the Postmaster General of Canada for the time being, and the contractor shall further take all reasonable and necessary precautions for the protection of such mails, while upon the said steamers or while in the contractor's charge or custody, from loss, damage or injury, in any way, and the contractors shall be responsible for any loss or damage thereto caused by negligence or want of proper care or accommodation on the part of the contractors, their agents or servants or on the part of the officers, employees or crew on board the said steamers, and this without regard to any question as to the legal liability of the Postmaster General to the owners of the articles of mail matter contained in such mails for damage or loss sustained in transit.

\section{Definition of term "Mails"}

The expression "mails" for the purpose of this contract shall be deemed to mean and include all boxes, bags, baskets or packets of or containing letters, post-cards, newspapers, parcels, books, or printed papers, and all other articles which under the Post Office Act and postal regulations for the time being in force are transmissible by post in Canada, without regard to place either of origin or destination, and also all empty bags, empty boxes and other receptacles, stores and articles used or to be used in carrying on the post office service, or which shall ordinarily be sent by or to or from the offices.

\section{No Letters except H. M. Mails to be Carried}

The contractors shall not, nor shall any of their agents or servants, or officers or craws of the said steamers receive or permit to be received on board of the said steamers any letters for conveyance other than those contained in His Majesty's mails, or which are or may be privileged by law, nor the mails of any other country, except such as are specified by the Postmaster General of Canada for the time being.

Government officials to be carried Free of Charge

The Honourable the Postmaster General of Canada, or the Honourable the Minister of Trade and Commerce for the time being, or any inspector or officer of the Post Office Department or the Department of Trade and Commerce, who may in the execution of his duty travel in the said steamers, shall be carried free of charge. 


\section{Proper accorints to be Kept}

The contractors shall keep full and proper accounts of and in connection with the working of this service, and shall keep such accounts separate and distinct from any other accounts of or connected with other branches of their business; and in any contingency which, in the opinion of the minister, may render such a course necessary, the contractors shall allow any officer or officers named by the minister free access to such accounts and all books, papers and documents connected therewith.

\section{Substitute for Disabled Steamers}

It is understood that if the said steamer shall be by peril of the sea or other unavoidable casualty, lost, destroyed or temporarily disabled from performing the voyages herein agreed to be performed according to the true intent and meaning of these presents, the contractors may in such case as soon as reasonably may be, having regard to the circumstances, replace the said steamer by another of equal class, speed, equipment, character and capacity to the satisfaction and approval of the minister in case the said steamer has been only temporarily disabled, and continue the service herein contracted for with such substituted or repaired steamer with as little delay as possible under all circumstances.

\section{Freight and Passenger Tariffs-Proof of Performance of Service to be Furnished}

The contractors shall carry on each steamer running under this contract, according to its capacity, on all voyages, all the freight and passengers which may be reasonably offered or obtained, and at tariff rates, both as to passengers and freight, which may be from time to time approved by the minister; and the contractors shall furnish to the minister such documents, information and evidence as may be required by the . minister to show the volume, extent and value of the trade carried on by the said steamer, and such customs certificates, documents and evidence as may be necessary or as may be required by the minister to prove the performance of the service herein contracted for, and to enable the minister to judge as to whether this contract is being carefully and faithfully carried out and performed and the furnishing of such certificates, documents, information and evidence, as hereinbefore specified, shall be a condition precedent to the payment of the subsidy herein provided for or any portion thereof.

\section{Deductions from Subsidy-Time-tables to be furnished-Docking Disabled Steamers}

Provided however, that it is the true intent and meaning of these presents that no amount or instalment of subsidy shall be payable or be paid at any time, unless it appears to the satisfaction of the minister that up to the time of such instalment becoming due, as herein stipulated, the service herein described and defined has been fully and faithfully performed, and that all provisions and stipulations as to freight and freight rates and dates of sailing have been in all respects faithfully observed and carried out, according to the true intent and meaning of these presents; and it is understood and agreed to be a further condition of these presents that the contractors shall at least two weeks prior to the first sailing under this contract furnish to the minister time-tables showing the proposed sailings, and upon the same being approved by the minister, they shall be duly advertised in such manner as he may direct; and it is also agreed that in case either of the steamers herein named, or a substituted steamer sanctioned by the minister, does not sail from a terminal port as herein specified within of the date fixed by such time-tables, there shall be deducted from the amount of subsidy payable for such voyage a sum equal to one-tenth of the amount otherwise payable for the performance of such voyage, and so in proportion for 
SESSIONAL PAPER No. 10a

further delays or failure to sail from such terminal port. Provided, however, that the minister may authorize any vessel to said either at an earlier or a later date than that specified in such time-tables should he for any reason deem it advisable to do so; it being understood and agreed that, in the event of any of the said steamers being at any time so disabled as to be obliged to be docked for repairs, the failure to perform the terms of this contract owing to such accident and for the time reasonably occupied in the repair of the damaged steamer, shall not be taken as a default or breach of the stipulations of this contract, or subject the contractors to deductions as above from the amount of this subsidy, if any, payable for any voyage delayed in consequence of such docking for repairs, but there shall be no claim for, nor payment of any subsidy in respect of any voyage not actually performed.

\section{Freight and Passenger Rates to be Approved by the Minister}

\section{(Inserted in contracts for ocean services only)}

The contractor shall at least three weeks prior to the first sailing under this contract, furnish to the minister a schedule of the freight rates proposed which schedule shall be subject to the approval of the Minister, and after being approved by him shall not be changed except with his consent; and the Minister may at any time, if he deem it advisable, fix the maximum rates to be charged between the different ports, on both east and westbound trips, on any article or class of goods, and the contractors shall carry between the ports hereinbefore named, on all voyages of the said steamships employed under the terms of this contract, all passengers or freight that may be offered or that can reasonably be procured, at rates which shall not be in excess of such maximum rates as fixed by the Minister, should he deem it advisable to so fix such maximum rates, and in no case shall any discrimination be made as regards rates, or otherwise directly or indirectly, against Canadian merchants, or shippers, who shall always have precedence for their freight and goods over all other merchants and shippers; and it is agreed and understood that the freight rates on eastbound trips, sailing from or as hereinbefore provided, on through bills of lading from any place in the provinces of Ontario and Quebec, or from any Canadian points farther west, shall in no case be greater than from the same place to via any United States route or port; and on the westbound trips the rates from to any place in Ontario or Quebec, or other Canadian points farther west, shall be as favourable as via any United States route or port to the same place; and it is further understood and agreed that the said steamers shall not carry between the ports of or and aforesaid, on any voyage run under the terms of this contract, either deals or lumber or timber to a greater extent than fifty per cent of the total quantity of the cargo carried on such voyage and such quantity only in case other Canadian products are not offering or cannot be obtained. Provided, however, that in the event of other cargo not being obtainable, satisfactory evidence of the fact being furnished to the Minister, then the contractors shall be allowed to make up the balance of the cargo with deals, boards or timber.

\section{Publicity of Tariff Charges}

The freight and passenger rates charged by the contractors over said route may at any time be required to be approved of by the Minister, whose decision shall be final, and the said freight and passenger rates shall be made available at all times to the public at the head office and the ageneies of the contractors.

$10 a-6$ 


\section{Calls at Foreign Ports}

The steamer employed in carrying out the provisions of this contract shall not on any of its trips call at any foreign port not specified in this contract.

\section{Carrying of nitro-glycerine or dangerous articles}

The contractors shall not convey or permit to be conveyed in any steamer while employed in this service any nitro-glycerine or any other article which in the opinion of the Minister shall be considered dangerous.

\section{Subsidy subject to Vote of Canadian Parliament}

It is conditioned, declared and agreed that the payment of subsidy, as hereinbefore stipulated, is subject to the amount specified being provided for the purpose by a vote of the parliament of Canada, and that if no amount is voted for the purpose, or if any amount voted has become exhausted in payment thereof, and no further sum is voted for the purpose, this contract or agreement shall terminate and become void and of no effect, and the party of the first part shall not in consequence be held liable to damage.

\section{Minister's Right to Terminate Contract}

It is declared to be the true intent and meaning of these presents, that the Minister shall have the right at any time during the continuance of this contract, upon 30 days' notice in writing to the contractors, their successors or assigns, to terminate this contract, and every matter and thing herein contained, if it shall appear to the Minister that there has been any breach on the part of the contractors, their successors or assigns, of any of the covenants, agreements, stipulations or provisions herein contained and entered into on the part of the contractors; and it is declared and agreed that the Minister shall at all times be the sole and final judge as to whether there has been any such breach, and his decision shall be absolute, final and conclusive.

\section{Assignment of Contract}

This contract shall not, nor shall any right or interest therein be assigned without the consent in writing of the Minister to such assignment having been first obtained.

Canadian Members of Parliament not Admitted to Share in Contract

It is a condition of these presents that no member of the House of Commons of Canada shall be admitted to any share or part of this contract or agreement nor to any benefit to arise therefrom.

\section{Changes in Contract}

The minister may authorize any change or changes in the terms of this contract as may not be consistent with the vote providing for the payment of the subsidy.

\section{Minister to be final judge as to full carrying out of contract-}

The minister shall at all times be the judge as to whether the terms of this contract have been or are being fully and faithfully carried out and compiled with within the true intent and meaning thereof, and his decision in that respect shall be binding, final and conclusive.

\section{Towing}

No towing shall be undertaken by the vessel performing the service specified in this contract, if such towing might interfere in any way with the regular performance of said service, except for the purpose of saving life or assisting vessels in distress, or performing other work of great importance, without the permission of the minister first having been obtained. 
SESSIONAL PAPER No. $10 a$

\section{Transportation of Trade Commissioners}

(Inserted in contracts for ocean services)

The Canadian Trade Commissioners and their wives, children and servants, or Canadian Commercial Agents, shall be granted free transportation, meals included, with first-class accommodation and free transportation for their household effects, upon any steamships employed by the contractors in the performance of this contract when requested so to do by the Minister, and when the said Commissioner or Commercial Agent is travelling upon his official duties or being transferred from one official post to another.

\section{Government Railway Clause}

(Inserted in contracts for Atlantic ocean services)

It is hereby agreed by the contractors that as the aid herein expressed and provided for by the Canadian Government is for the express purpose of encouraging the development of Canadian trade and the transportation of Canadian goods through Canadian channels the Company accepts the aid on these conditions, and agrees that all freight booked or carried by the said steamships from a port in the United Kingdom and during the time these make Halifax or St. John their terminal port, shall, when not otherwise expressly routed by shippers or consignees, be delivered to the Canadian National Railways at Halifax or St. John for shipment to final destination in Canada, provided that the rates demanded by the Canadian National Railways shall not be in excess of the rates charged by any other railway company from said ports to final destination in Canada.

(a) Contractors are to hand over to the Canadian National Railways at Halifax pessengers for points in the Maritime Provinces or the Province of Quebec, providing the route of such passengers is controlled by the contractors and that they are not otherwise routed. It is further agreed that the contractors through their agents in Canada shall see that they have all such freight for export as may be secured by them for a port in the United Kingdom at which the subsidized line may call delivered to the Canadian National Railway at Montreal.

\section{Calls at Government Wharves}

(Inserted in contracts for local services)

In consideration of the subsidy herein stipulated the contractors agree to call at all Government wharves when such is practicable and when such wharves are available.

\section{Handling of perishable products}

(Inserted in contracts for Atlantic ocean services)

The handling, loading, stowing and unloading of any fruit or perishable products carried by the said vessels shall be subject to and under the supervision of any cargo inspector or other officer appointed for that purpose, should the Minister of Agriculture for Canada deem it advisable. 


\section{INDEX TO SERVICES}

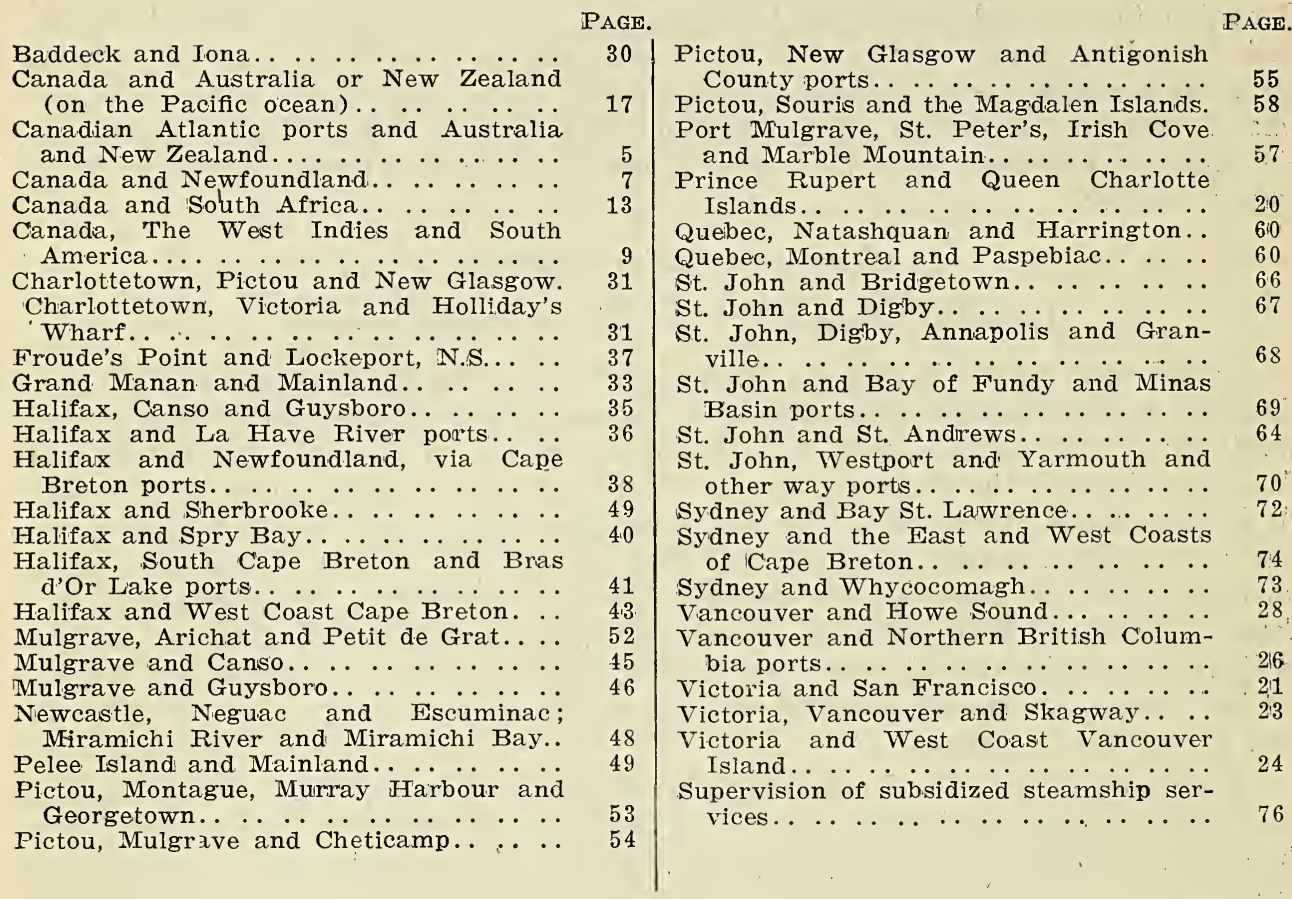




\section{INDEX TO PERSONS OR COMPANIES SUBSIDIZED}

\begin{tabular}{|c|c|c|}
\hline & & $A G B$ \\
\hline 7 & Arand Trunk Pacific Coast SS. Co.. & \\
\hline 30 & alifax and Canso SiS. Co.. & \\
\hline & Halifax and Inverness SS. Co. & \\
\hline 73 & alifax and Sheet Harbour sS. Co... & 0 \\
\hline 66 & 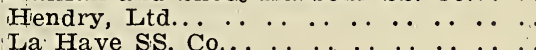 & \\
\hline 67 & eslie, William C........... & \\
\hline & t, N.S., Town of. . & \\
\hline 23 & SS. Co... . . . & \\
\hline & al, Roderick. . . . . . & \\
\hline 24 & $\begin{array}{l}\text { Mclure, Wm.. } \\
\text { Miramichi Steam Navigation Co., Ltd. }\end{array}$ & \\
\hline 45 & 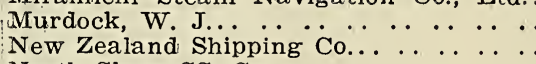 & \\
\hline 70 & Sis. Co... & 72 \\
\hline 31 & & \\
\hline 60 & nd, Ltd.. & \\
\hline 74 & ship Co... & 57 \\
\hline 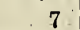 & Steam Packet Co... & \\
\hline 67 & W... & \\
\hline 31 & SS. Co... & \\
\hline 52 & $\cdots$ & \\
\hline & of New Zealand....... & 17 \\
\hline 1 & & 68 \\
\hline & in & \\
\hline 5 & e Island Steamshir & \\
\hline 33 & & 49 \\
\hline
\end{tabular}

Anchor-Donaldson Line. . . . . . . . . . Baddeck SS. Co., Ltd.. . . . . . . . Bay of Fundy and Minas Basin SS. Co.

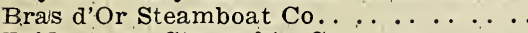
Bridgetown Steamship Co... . . . . Canadian Pacific Railway (St. John and Digby) ... . . . . . . . . . Canadian Pacific Railway (Victoria Viancouver and Skagtway).........

Canadian Pacific Railway (Victoria and West Coast Vancouver Island)...... . Cann \& Son, Hugh (Mulgrave and

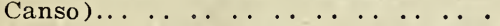
Cann \& Son, Hugh (St. John and Westport )...................... Charlottetown SS. Co. . . . . . . . . Clarke Bros. . . . . . . . . . . . . . . Coastal Steamship Co...........

Cunard Line................. Dominion Atlantic Railway Co.. ....

Eastern Transport, Ltd.. . . . . . . . . Elaline SS. Co... . . . . . . . . . . . . . Elder-Dempster \& Co. (South African

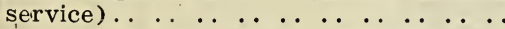
Farquhar \& Co., J. A. (Halifax and Newfoundland via Cape Breton)... . . Georgetown SS. Co............. Grand Manan Steamboat Co........
PAge. 
INDEX TO NAMES OF VESSELS EMPLOYED

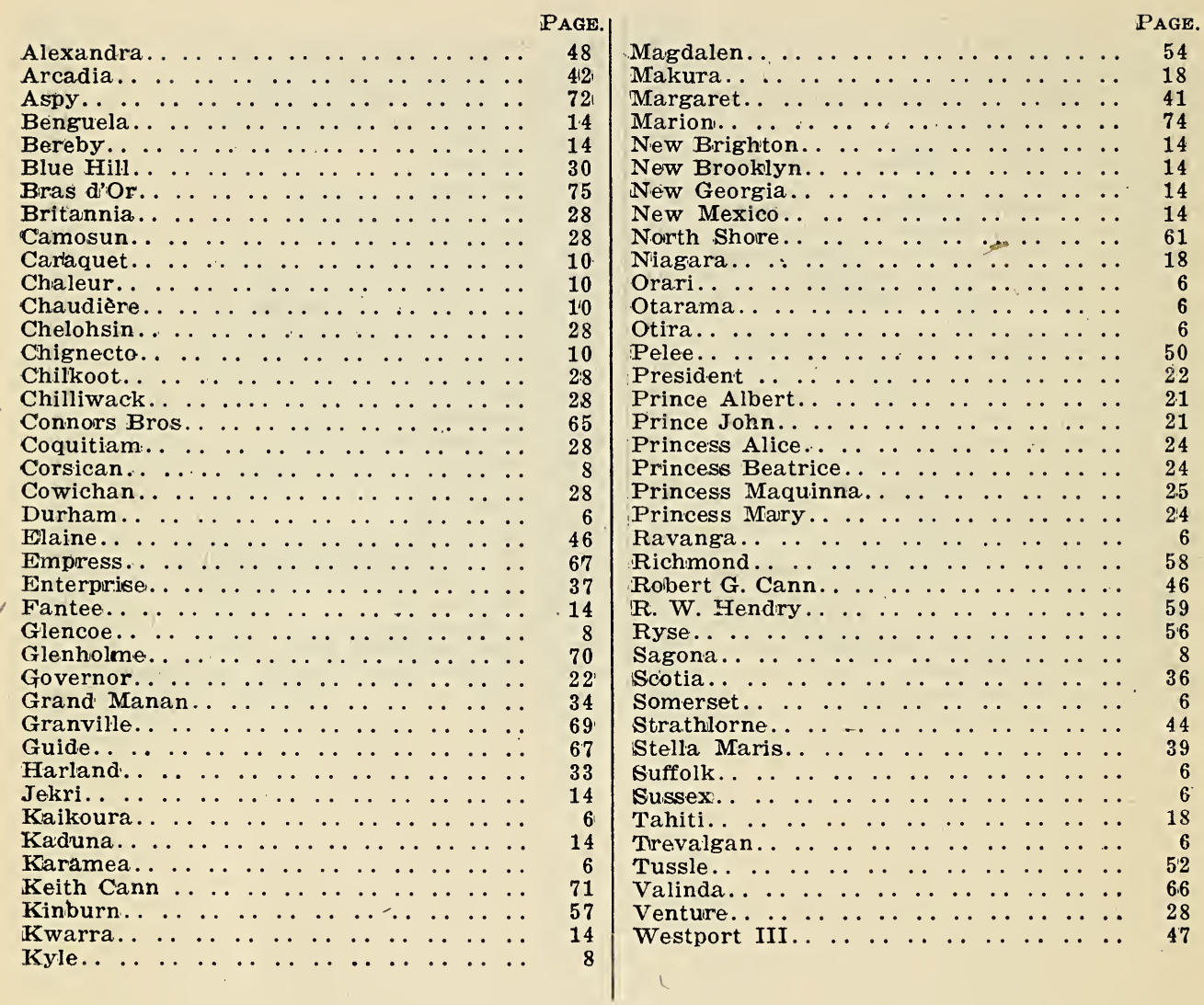




$\frac{1}{2}+9$

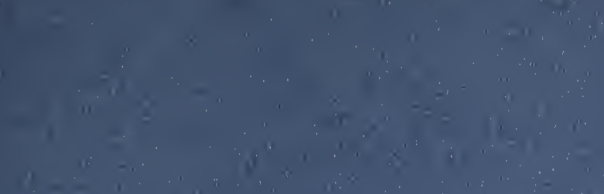

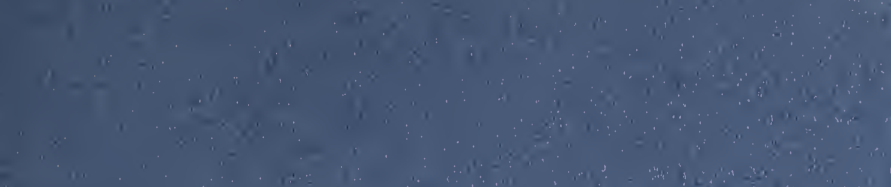

(1) 40

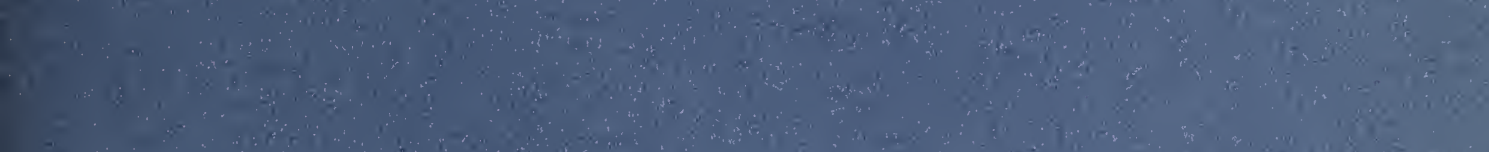
$40+x^{2}$

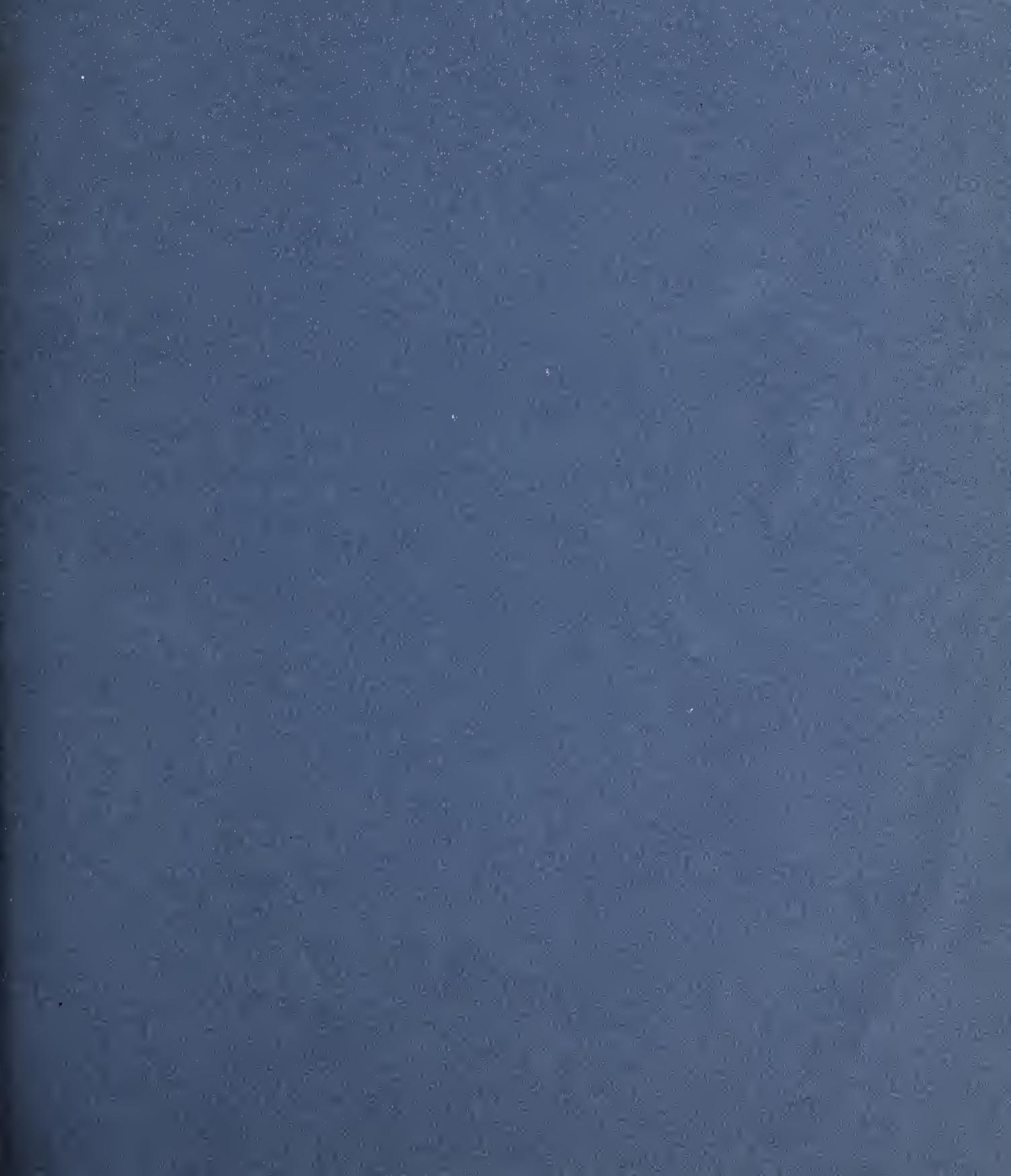




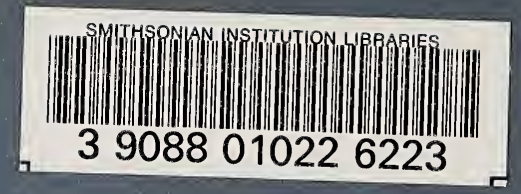




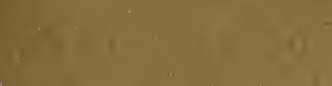

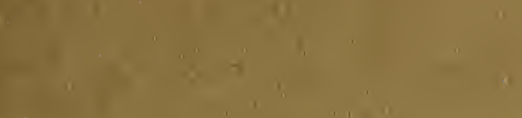

a

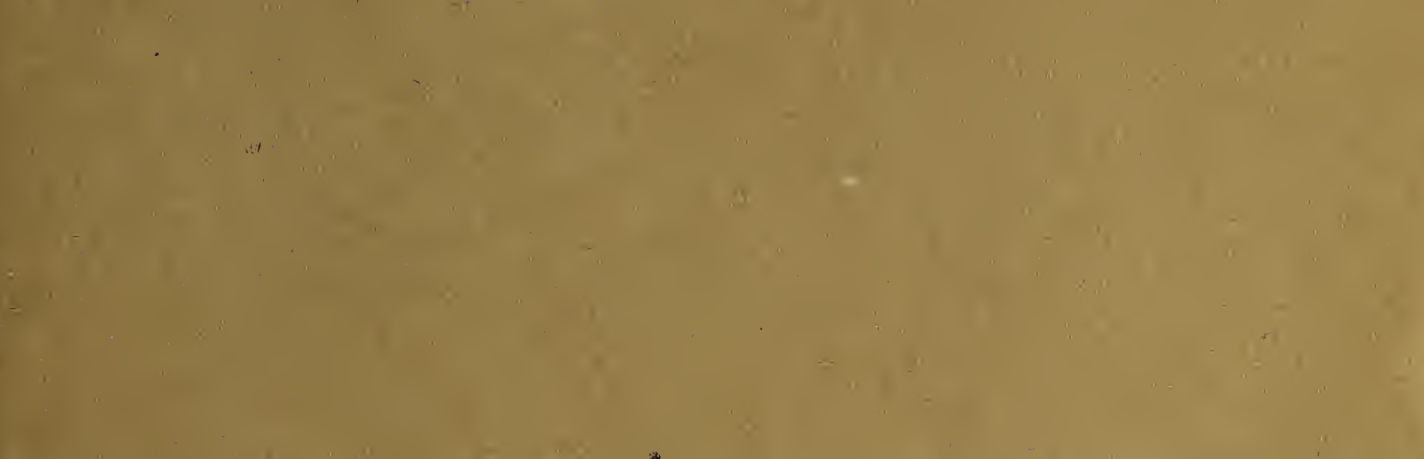

is

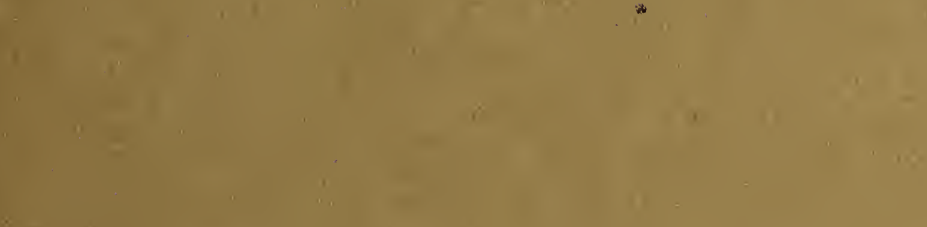

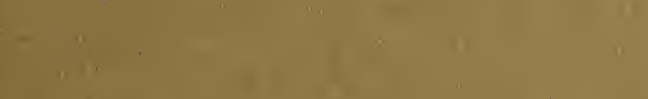

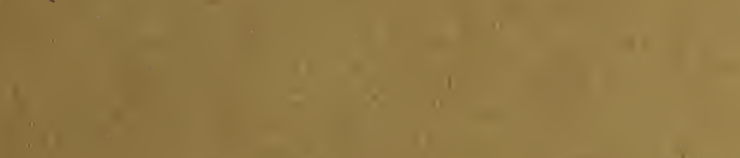

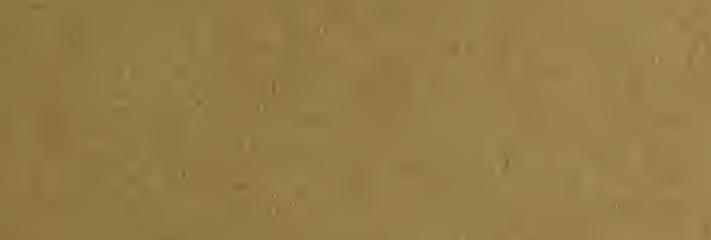

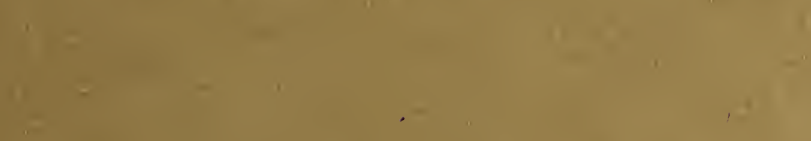

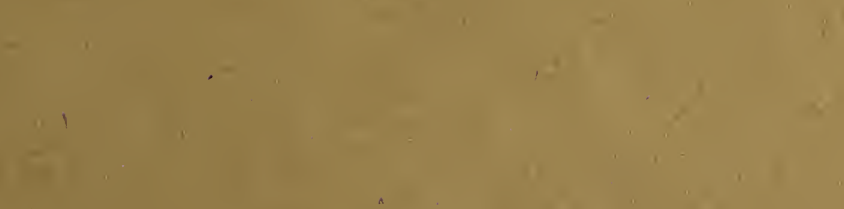

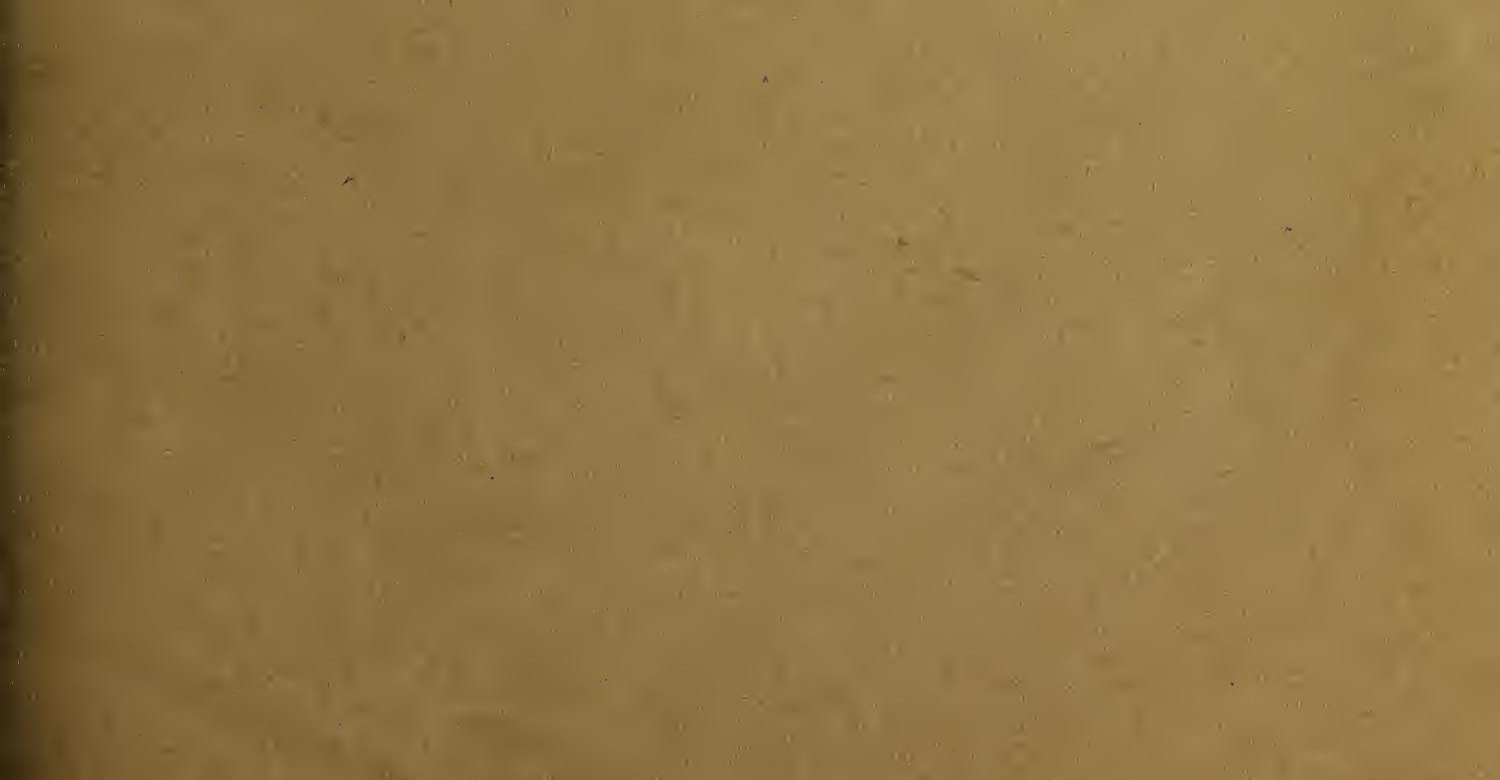


